\title{
Räumliche Mobilität am Arbeitsmarkt Einfluss- und Erfolgsfaktoren überbetrieblicher und überregionaler Mobilitätsprozesse
}

\author{
Dissertation \\ zur Erlangung des akademischen Grades eines Doktors \\ der Sozial- und Wirtschaftswissenschaften \\ der Sozial- und wirtschaftswissenschaftlichen Fakultät \\ der Otto-Friedrich-Universität Bamberg
}

\author{
vorgelegt von \\ Franziska Ganesch, M. A.
}

Bamberg, 2019 
URN: urn:nbn:de:bvb:473-irb-477456

DOI: https://doi.org/10.20378/irb-47745

Erstgutachter: Prof. Dr. Olaf Struck

Zweitgutachterin: Prof. Dr. Henriette Engelhardt-Wölfler

Beisitzerin: Prof. Dr. Sandra Buchholz

Mündliche Prüfung: 20.03.2020 


\section{Vorwort}

Zahlreiche Menschen haben mich während des Entstehungsprozesses meiner Dissertation begleitet, denen ich an dieser Stelle danken möchte. Mein erster Dank gilt meinem Doktorvater Prof. Dr. Olaf Struck, der als Erstgutachter den Entstehungsprozess dieser Arbeit wissenschaftlich begleitet hat und während der gesamten Zeit mein wichtigster Ansprechpartner war. Ich habe in der Zusammenarbeit und den fachlichen Diskussionen nicht nur viel von ihm gelernt, seine wissenschaftliche Herangehensweise hat mich sicher auch weit über diese Arbeit hinaus geprägt. Dankbar bin ich auch für die Freiheiten, die er mir bei der Verfolgung meiner wissenschaftlichen Ziele eingeräumt hat und das Vertrauen, das er mir dabei entgegengebracht hat. Zu Dank verpflichtet bin ich darüber hinaus meiner Zweitgutachterin, Frau Prof. Dr. Henriette Engelhardt-Wölfler, sowie der Beisitzerin meiner Promotionskommission, Frau Prof. Dr. Sandra Buchholz, für ihr Interesse an meiner Forschungsarbeit und ihren wertvollen Hinweisen.

Meinen Kolleginnen und Kollegen an der Professur für Arbeitswissenschaft danke ich von Herzen für den freundschaftlichen Rat und die fortwährende Bestärkung von Beginn an. Mein ganz besonderer Dank gilt Matthias Dütsch, ohne dessen Anstoß dieses Projekt gar nicht entstanden wäre, für die unzähligen Ratschläge, Hinweise und Kritik, die mich und diese Arbeit in vielerlei Hinsicht inspiriert und bereichert haben. Besonders möchte ich auch meiner Familie und meinem Freund für die stete Unterstützung danken.

Bamberg, im Dezember 2019

Franziska Ganesch 


\section{Aufbau der Arbeit ${ }^{1}$}

Überbetriebliche und überregionale Mobilität auf dem Arbeitsmarkt:

Theoretische Konzepte und empirische Befunde

Franziska Ganesch

Employment trajectories in heterogeneous regions: Evidence from Germany

Matthias Dütsch, Franziska Ganesch und Olaf Struck

Regionale Mobilität am Arbeitsmarkt. Individuelle, betriebliche und wirtschafts-trukturelle Determinanten von Mobilität und Einkommen

Franziska Ganesch, Matthias Dütsch und Olaf Struck

Regional Mobility and Spatial Inequality: Determinants of Spatial Labor Market Behavior Considering Firm- and Region-Specific Factors

Franziska Ganesch

Arbeitsplatzmobilität zwischen Ost-, Nord-, und Süddeutschland:

Erfolgsfaktoren von Einkommenszuwächsen

Franziska Ganesch, Matthias Dütsch und Olaf Struck

\footnotetext{
${ }^{1}$ Ich weise darauf hin, dass die einzelnen Bestandteile der kumulativen Dissertation - mit Ausnahme des Rahmenpapiers „Überbetriebliche und überregionale Mobilität auf dem Arbeitsmarkt: Theoretische Konzepte und empirische Befunde“ - nicht vervielfältigt werden dürfen und bitte darum, diese Beiträge im Original zu zitieren.
} 


\title{
Überbetriebliche und überregionale Mobilität auf dem Arbeitsmarkt: Theoretische Konzepte und empirische Befunde
}

\author{
Franziska Ganesch
}

\section{Inhaltsverzeichnis}

1 Einleitung 2

2 Arbeitsmarkttheoretische und regionalökonomische Ansätze und ihre Befunde 6

$\begin{array}{lr}2.1 \text { Beschäftigungsstabilität und Betriebsmobilität } & 6\end{array}$

2.2 Befunde zum räumlichen Arbeitsmarktverhalten 11

2.3 Befunde zur Einkommensentwicklung im Zuge von Betriebsmobilität 16

$\begin{array}{lll}2.4 & \text { Zwischenfazit } & 18\end{array}$

3 Theoretisches Modell und methodische Umsetzung 19

3.1 Theoretisches Modell: Entscheidung zu (überregionaler) Betriebsmobilität 19

$\begin{array}{ll}3.2 & \text { Methodische Umsetzung } \\ & 26\end{array}$

$4 \quad$ Darstellung und Interpretation der eigenen Erkenntnisse 29

4.1 Wirtschaftsgeografische Ausgangslage 29

4.2 Erkenntnisse aus dem Fachartikel „Employment trajectories in heterogeneous regions: Evidence from Germany“"

4.3 Erkenntnisse aus dem Fachartikel „Regionale Mobilität am Arbeitsmarkt. Individuelle, betriebliche und wirtschaftsstrukturelle Determinanten von Mobilität und Einkommen“ 34

4.4 Erkenntnisse aus dem Fachartikel „Regional Mobility and Spatial Inequality: Determinants of Spatial Labor Market Behavior Considering Firm- and Region-Specific Factors“"

4.5 Erkenntnisse aus dem Fachartikel „Arbeitsplatzmobilität zwischen Ost-, Nordund Süddeutschland: Erfolgsfaktoren von Einkommenszuwächsen“" 38

$5 \quad$ Zusammenfassung und Fazit 42

\section{Abbildungsverzeichnis}

Abbildung 1: Regionale Disparitäten 29

$\begin{array}{ll}\text { Anhang } & 48\end{array}$

Literaturverzeichnis $\quad 53$ 


\section{Einleitung}

\section{Einleitung}

Soziale und wirtschaftliche Anpassungsprozesse sind von Flexibilität und Mobilität gekennzeichnet (Nisic und Abraham 2014, S. 675). Im Rahmen der Diskussion um wettbewerbsnotwendige Anpassungen von Arbeit und Arbeitsmärkten wird unter anderem die Bereitschaft zu überbetrieblicher bzw. räumlicher Mobilität von Arbeitskräften erwartet (Doering-Manteuffel 2007, S. 575; Doering-Manteuffel und Raphael 2011, S. 38; vgl. auch Sennett 2006). Der Niedergang von Wirtschaftszweigen, wie dem Bergbau, Schiffbau und der Textilindustrie, aber auch die Flexibilisierung und Spezialisierung von Arbeit führten dazu, dass für jeweilige Berufsgruppen qualifikationsadäquate Arbeitsplätze nicht mehr bzw. nicht mehr allerorts zu finden sind. So kennzeichnet ein hohes Maß an Mobilität Arbeitsmärkte bereits seit den 1970er Jahren (Struck 2006, S. 15f.). Vor allem in den 1990er Jahren werden durch den Einsatz betrieblicher Flexibilisierungsstrategien Destabilisierungs- und Destandardisierungstendenzen auf dem Arbeitsmarkt in Deutschland festgestellt (Blossfeld et al. 2006; Buchholz 2008; Dombois 1999; Dörre 2005a, b), die dann auch zumindest für bestimmte Beschäftigtengruppen große Erwerbsrisiken in sich bergen (Dütsch und Struck 2014).

Zwischenbetriebliche bzw. räumliche Mobilität ist demnach eine wesentliche Anforderung für das Leben in modernen Gesellschaften (Giddens 1991; Kley 2016; Sennett 1998; Urry 2007). Bewegungen von Individuen und Haushalten im geografischen Raum sind kein grundsätzlich neues Phänomen, wie der historische Blick auf Wanderungsbewegungen in Deutschland verdeutlicht (Bade und Oltmer 2007). Lediglich die Häufigkeit von Mobilitätsereignissen und deren Konsequenzen für andere Lebensbereiche führten im Laufe der Zeit zu veränderten mobilen Lebensformen (Schneider et al. 2002a, 2009). So konstatiert Tully (2006, S. 15), „,[z]ur keiner Zeit war die Mobilität des modernen Menschen derart extensiv“.

Anlass und Ausgangspunkt mobiler Lebensformen sind oftmals die Erwerbskarriere bzw. erwerbsbezogene Ziele oder Erfordernisse (Schneider et al. 2016). Denn insbesondere Erwerbsarbeit bestimmt die Lebensverläufe von Individuen (Kohli 1985; Mayer 2009). In modernen Wohlfahrtsgesellschaften strukturieren Arbeitsmärkte maßgeblich die Zuteilung von Lebenschancen (Abraham und Hinz 2008; Kreckel 1983, S. 140; Vobruba 1990, 2000). Dies betrifft zum einen das Erwerbseinkommen, zum anderen damit einhergehende Transferzahlungen in das soziale Sicherungssystem. 


\section{Einleitung}

In der Bundesrepublik Deutschland bestehen u. a. infolge der Wiedervereinigung und des ostdeutschen Transformationsprozesses Unterschiede hinsichtlich ökonomischer Bedingungen und Beschäftigungschancen (Albrech et al. 2016). In der Dekade 2000 war das Arbeitslosigkeitsniveau in Ostdeutschland im Durchschnitt doppelt so hoch wie in den westdeutschen Bundesländern. Das durchschnittliche Lohnniveau in Ostdeutschland ist bis heute deutlich geringer als in Westdeutschland (Arntz 2010, S. 427; Spellerberg 2016, S. 355). Dies gilt ungeachtet der anfänglich erheblichen Angleichungsprozesse in Ostdeutschland nach der deutschen Wiedervereinigung (Struck 2017) sowie der seit längerem positiven Entwicklung auf dem Arbeitsmarkt (Eichhorst et al. 2017). Daneben existiert ein wirtschaftsstrukturelles Süd-Nord-Gefälle (Bohler und Hildenbrand 2006; Statistisches Bundesamt 2016) sowie kleinräumigere Disparitäten (BBSR 2016; Sixtus et al. 2019). Fortbestehende ökonomische Ungleichheiten zwischen Regionen sind nicht selbstverständlich. So besteht in Artikel 72 im Grundgesetz ein fortwährender Verfassungsauftrag für den Gesetzgeber „gleichwertige Lebensverhältnisse“ in allen Teilen des Landes anzustreben. Aus neoklassischer Perspektive betrachtet wäre zu erwarten, dass es durch Bewegungen von Individuen und Haushalten im Raum (regionale und überregionale Arbeitgeberwechsel) zu einem Gleichgewicht des Arbeitsangebots und der Arbeitsnachfrage und damit zu einer Angleichung regionaler Arbeitslosigkeitsniveaus kommt. Dadurch, dass Regionen mit einer hohen Arbeitslosigkeit in Richtung prosperierender Räume verlassen werden (Buch 2006, S. 60).

Wird Mobilität zwischen Regionen beobachtet, dann sind individuelle berufliche und private Ziele und Präferenzen sowie deren Kontextabhängigkeit neben makroökonomischen Angleichungsprozesse bedeutsam. Diese können sowohl Hemmnisse als auch Treiber für (überregionale) Betriebswechsel sein und somit zu selektiven Wanderungsmustern führen. Doch welches sind die individuellen, betrieblichen und regionalen Determinanten von (überregionaler) Betriebsmobilität? Unter welchen Bedingungen ist regionale und überregionale Betriebsmobilität einkommenserfolgreich?

Vor diesem Hintergrund untersucht mein Dissertationsprojekt „Räumliche Mobilität am Arbeitsmarkt" die individuellen, betrieblichen und regionalen Einflüsse von Erwerbsverläufen im Hinblick auf betriebliche Beschäftigungsstabilität, intra- und interregionaler Betriebswechsel und deren Folgen für Einkommenschancen. Bestehende Analysen von Erwerbsverläufen fokussierten insbesondere individuelle Charakteristika (Bender et al. 2000; Boockmann und Steffes 2010; Fitzenberger und Garloff 2007; Grotheer et al. 2004; Struck et al. 2007). Beschäftigungsverhältnisse 


\section{Einleitung}

sind jedoch darüber hinaus in betriebliche Opportunitätsstrukturen und regionale Kontexte eingebunden. Betriebsmerkmale wurden jedoch bislang sowohl bei den vergleichsweise wenigen Untersuchungen zu der Frage, welche Gruppen sind (überregional) betriebsmobil, wie auch bei der Analyse von „Renditen“ (räumlicher) Betriebsmobilität kaum berücksichtigt.

Mit Rückgriff auf segmentationstheoretische Überlegungen (Doeringer und Piore 1971; Sengenberger 1987) und darauf aufbauende Ansätze zu betrieblichen Beschäftigungssystemen (Hendry 2003; Struck und Dütsch 2012) konnten bislang personal- und qualifikationsstrukturelle sowie branchen- und betriebsgrößenspezifische Effekte auf die Beschäftigungsstabilität nachgewiesen werden (Bender et al. 2000; Boockmann und Steffes 2010; Grotheer et al. 2004; Struck 2006; Struck et al. 2007). Diese Forschungen geben Hinweise darauf, dass betriebsstrukturelle Faktoren individuelle Erwerbs- und Einkommenschancen beeinflussen. Die regionale Wirtschaftsstruktur und die damit einhergehende räumliche Segmentierung von Arbeitsmärkten (Fassmann und Meusburger 1997) werden in erster Linie in regionalökonomischen, makrotheoretischen Analysen zur Beschäftigungsentwicklung untersucht. Forschungsarbeiten zu individuellen Erwerbsverläufen beziehen heterogene raumstrukturelle Aspekte bislang jedoch selten systematisch mit ein.

Daran knüpft das vorliegende Forschungsprojekt an. Es verbindet arbeitsmarkttheoretische Ansätze und Überlegungen des ,new structuralism“ (Baron und Bielby 1980) mit regionalökonomischen Überlegungen (Fujita et al., 2001; Krugman 1991, 1998), um die Heterogenität der Arbeitsmarktbedingungen über Erklärungsfaktoren auf der Mikro-, Meso- und Makroebene hinweg abzubilden und den Analysefokus dabei speziell auf strukturelle Einflussfaktoren zu lenken. Für dieses Vorhaben wurde ein Linked-Employer-Employee Datensatz (LM9310) des Instituts für Arbeitsmarkt und Berufsforschung (IAB) herangezogen. Dieser wurde um Informationen zu regionalen Strukturindikatoren auf der Ebene von Raumordnungsregionen ergänzt.

Die vorliegende Synopsis ist folgendermaßen strukturiert: Zunächst werden arbeitsmarkttheoretische und regionalökonomische Ansätze und ihre Befunde - auch im Hinblick auf Einkommensveränderungen im Zuge von Betriebsmobilität - skizziert (Kapitel 2) und in ein akteursbezogenes theoretisches Entscheidungsmodell zu Betriebsmobilität überführt (Kapitel 3). In diesem Rahmen wird das Konzept der Raumordnungsregionen als das der Untersuchung zugrundeliegende räumliche Analyseraster vorgestellt. Weiterhin werden in Kapitel 4 zunächst die wirtschaftsgeografische Ausgangslage beschrieben sowie die in vier Fachzeitschriften publizierten Erkenntnisbei- 


\section{Einleitung}

träge zu Betriebsmobilität dargestellt und interpretiert. Abschließend werden die Befunde hinsichtlich ihres Beitrages zur Arbeitsmarkt- und Lebensverlaufsforschung reflektiert sowie Anknüpfungspunkte für zukünftige Forschung im Bereich regionaler und überregionaler Betriebsmobilität dargelegt. 


\section{Arbeitsmarkttheoretische und regionalökonomische Ansätze und ihre Befunde}

\subsection{Beschäftigungsstabilität und Betriebsmobilität}

In Untersuchungen zur Mobilität zwischen Betrieben wird der Blick häufig auf sozio-demografische Merkmale gerichtet. Insbesondere hinsichtlich individueller Determinanten der Beschäftigungsstabilität liegen entsprechend den humankapitaltheoretischen Annahmen (Becker 1975) bereits zahlreiche Befunde vor. Demnach variiert die Produktivität eines Arbeitnehmers mit dem Umfang seiner Qualifikationen sowie dessen Fähigkeiten und Hochschulabsolventen sowie Facharbeiter sind im Vergleich zu un- oder angelernten Arbeitskräften stabiler beschäftigt (Bender et al. 2000; Grotheer et al. 2004; Struck et al. 2007). Zudem weisen insbesondere Hochschulabsolventen im Vergleich zu Geringerqualifizierten eine höhere freiwillige und tendenziell erfolgreichere überbetriebliche Mobilität mit Karriereaufstiegen auf, während gering qualifizierte Arbeitskräfte nach dem Ausscheiden aus einem Betrieb Schwierigkeiten haben, eine Anschlussbeschäftigung zu finden und das bisherige Lohnniveau zu halten (Boockmann und Steffes 2010; Dütsch et al. 2013; Grotheer et al. 2004; Hacket 2009). Auch das Geschlecht - Männer sind stabiler beschäftigt als Frauen - und das Alter - insbesondere jüngere Beschäftigte sind betriebsmobil haben einen Effekt auf die Erwerbs- und Karrierechancen (ebd.). Zudem sind Erwerbskarrieren von Migranten auch bei Kontrolle von soziodemografischen Merkmalen von einer geringeren betrieblichen Stabilität sowie von längeren und häufiger auftretenden Arbeitslosigkeitsphasen geprägt (Kogan 2004, 2011). Eine hohe Relevanz wird darüber hinaus der Zugehörigkeit zu spezifischen Arbeitsmarkteintrittskohorten sowie dem vorherigen Erwerbsstatus beigemessen. Demnach bilden die einzelnen Phasen und Übergänge im Erwerbsverlauf einen „,endogenen Kausalzusammenhang“ (Blossfeld 1985; Mayer und Blossfeld 1990). Die zu unterschiedlichen Zeitpunkten im Erwerbsleben gegebenen Chancen und Risiken sind dann das Resultat kumulativer Prozesse (ebd.; Bender et al. 2000; Manzoni 2012; Struck 2006). Dementsprechend verringern diskontinuierliche Erwerbsverläufe die Chancen auf dem Arbeitsmarkt und erhöhen die Arbeitslosigkeitsrisiken sowie im Falle von überbetrieblicher Mobilität die Einkommensverlustrisiken (Bender et al. 2000; Boockmann und Steffes 2010; Hacket 2009).

Erwerbsverläufe und Mobilitätsprozesse resultieren allerdings immer auch aus der Interaktion von Arbeitgebern und Arbeitnehmern, wobei sie in der Literatur häufig auf betrieblich induzierte 
Segmentierung des Arbeitsmarktes im Kontext institutioneller Rahmenbedingungen zurückgeführt werden (Doeringer und Piore 1971; Sengenberger 1987). Hierauf aufsetzende neuere Ansätze etwa zu HRM-Systems oder betrieblichen Beschäftigungssystemen leiten Segmentierungsprozesse aus innerbetrieblichen Arbeits- und Beschäftigungssystemen ab (Hendry 2003; Köhler et al. 2008; Struck und Dütsch 2012). Es wird - teilweise mit Bezug auf Transaktionskostenansätze - argumentiert, dass Betriebe aufgrund der betrieblichen Ausrichtung der Arbeitsorganisation, Technik und qualifikatorischen Arbeitsanforderungen unter Berücksichtigung institutioneller Normen und mit Blick auf die auf internen und externen Märkten verfügbaren Arbeitskräfte mit unterschiedlichen Beschäftigungssystemen operieren. Diese unterscheiden sich hinsichtlich der Dauer von Beschäftigungsverhältnissen und in der Öffnung gegenüber externen Arbeitsmärkten. Forschungsarbeiten zu diesem „new structuralism“ (Baron und Bielby 1980, S. 737) können personal- und qualifikationsstrukturelle, arbeitsorganisatorische sowie branchen- und betriebsgrößenspezifische Effekte auf den Erwerbsverlauf mittels kombinierter Individual- und Betriebsdaten nachweisen (Bender et al. 2000; Boockmann und Steffes 2010; Dütsch und Struck 2014; Grotheer et al. 2004; Struck 2006). Die genannten Arbeiten stellen die Bedeutsamkeit eines knappen spezifischen Humankapitals, der betrieblichen Aus- und Weiterbildung, der Existenz eines Betriebsrates und von Investitionen in neue Technologien für die (betriebliche) Beschäftigungsstabilität heraus (ebd.). Diese Bedingungen treffen auf Beschäftigte in Betrieben des verarbeitenden Gewerbes vergleichsweise häufiger zu. Für Arbeitskräfte im Dienstleistungsbereich, welche Tätigkeitsprofile mit geringeren betriebs- oder tätigkeitsspezifischen Qualifikationsanteilen haben, bestehen gegenüber Arbeitskräften in anderen Wirtschaftsbereichen höhere Betriebswechselchancen bei gleichzeitig geringeren Arbeitslosigkeitsrisiken (Struck 2006, S. 153).

Die gängigen arbeitsmarkttheoretischen Ansätze lassen makrostrukturelle Faktoren in ihren Erklärungen weitgehend unberücksichtigt (Fujita et al. 2001). Regionalökonomische Forschungsbeiträge, deren Bedeutung in den letzten Jahren innerhalb der Wirtschaftswissenschaften insbesondere durch Krugmans Beiträge zur „New Economic Geography“ (Krugman 1991, 1998) zugenommen haben (Fujita et al. 2001), betonen jedoch den Stellenwert des geografischen Raumes für das regionale Wachstum und fokussieren auf die Erklärung regionaler Heterogenitäten. Krugman (1991) entwickelte dazu ein Zentrum-Peripherie-Modell, das in Anlehnung an Hirschman (1958), auf der Idee divergierender zentripetaler und zentrifugaler Kräfte aufbaut. Es bezieht die Wirkung positiver externer Effekte ein und stellt die wechselseitige Relation von Skalenerträgen, 
Transportkosten sowie Wanderungen heraus. Demnach führen zentripetale Kräfte zu Urbanisierungseffekten, da sie eine Konzentration ökonomischer Aktivitäten im geografischen Raum bewirken. Industrielle Zentren werden dadurch gestärkt, dass Betriebe und Arbeitskräfte Agglomerationsvorteile ausnutzen. Ein Betrieb versucht im Falle hoher Skalenerträge, die Produktion auf einen einzigen Standort zu beschränken und bedient von dort aus den Markt. Um Transportkosten zu vermeiden, wird sich der Betrieb in einer Region mit einer großen Nachfrage, d.h. einer hohen Bevölkerungsdichte, ansiedeln. Dadurch wiederum werden Arbeitskräfte und weitere Betriebe attrahiert, die ihrerseits das Ziel verfolgen, Agglomerationsvorteile infolge eines großen Absatzmarktes oder eines großen Arbeitskräftepools in dieser Region zu realisieren (Krugman 1991). Folglich differenzieren sich ökonomische Zentren und Peripherien umso wahrscheinlicher aus, je geringer die Transportkosten ausfallen und je höher die Skalenerträge sind. Gemäß Fassmann und Meusburger (1997) bildet sich dann ausgehend von einer stabilen Güternachfrage und der Ansiedlung leistungsfähiger Unternehmen ein primäres Arbeitsmarktsegment in zentralen Räumen aus, das durch stabile Arbeitsplätze, gute Bezahlung, Aufstiegschancen sowie vorwiegend gut qualifizierte Arbeitskräfte gekennzeichnet ist. In peripheren Räumen entwickelt sich hingegen aufgrund der instabilen Nachfrage und weniger leistungsstarker Unternehmen ein sekundäres Arbeitsmarktsegment mit instabilen und gering bezahlten Arbeitsverhältnissen, geringen Qualifikationsanforderungen und wenigen Aufstiegschancen. Tatsächlich kann die neuere regionalökonomische Forschung eine stärkere Arbeitskräftenachfrage in dicht besiedelten Gegenden nachweisen (Blien et al. 2006; Farhauer und Granato 2006). Dichter besiedelte Räume weisen mehr und bessere Beschäftigungsmöglichkeiten insbesondere in konjunkturellen Phasen des Aufschwungs auf. In ländlichen Regionen haben Beschäftigte während konjunktureller Abschwünge ein größeres Arbeitslosigkeitsrisiko (Dütsch und Struck 2014, S. 119).

Ein weiterer Ansatz der Regionalforschung, die endogene Wachstumstheorie, stellt einen Zusammenhang zwischen der regionalen Qualifikationsstruktur der Beschäftigten und dem Wachstumspotential her und negiert dadurch die Annahme der neoklassischen Modelle, dass in der langen Frist das Wachstum exogen bestimmt ist (Lucas 1988; Romer 1986). Dabei rekurriert die endogene Wachstumstheorie auf Arbeiten zum Humankapital und verweist auf die Abhängigkeit des Wachstumspotentials einer Region von dem dort verfügbaren Bestand an Qualifikationen und Wissen. Demnach geht eine steigende Humankapitalausstattung mit einer steigenden Produktivität der Beschäftigten einher, wobei eine Konzentration von Humankapital als „Wachstumsmotor“ 
für eine Region auch bei konstanter Technologie dient. Forschungsarbeiten zur Wirkung der regionalen Qualifikationsstruktur konnten nachweisen, dass das Beschäftigungswachstum einer Region insbesondere von einer berufsfachlich qualifizierten bzw. hochqualifizierten Beschäftigtenstruktur positiv beeinflusst wird (Blien und Wolf 2002; Farhauer und Granato 2006). Schlitte et al. (2010) ermitteln anhand des IAB-Betriebspanels für Westdeutschland im Zeitraum von 1993 bis 2005 eine zunehmende Segregation nach Qualifikationen in den Raumordnungsregionen (siehe auch Gerlach et al. 2002). Dies ruft darüber hinaus eine divergente Entwicklung hinsichtlich der Beschäftigung sowie der Löhne hervor. Allerdings ist die qualifikatorische Segregation wirtschaftsstrukturell unterschiedlich stark ausgeprägt. Während Regionen, die sich auf den Sektor des verarbeitenden Gewerbes spezialisiert haben, eine weniger stark ausgeprägte Segregation aufweisen, ist in Regionen mit einer großen Bedeutung des Dienstleistungssektors das Gegenteil zu beobachten. Zudem hat ein hoher Anteil gut qualifizierter Beschäftigter in einer dicht besiedelten Region eine segregationsfördernde Wirkung. Anhand von Betriebs- sowie auch Beschäftigtendaten konnte ermittelt werden, dass ein großer Anteil hoch qualifizierter Arbeitskräfte das Beschäftigungswachstum in einer Region fördert (Schlitte et al. 2010; Shapiro 2006; Südekum 2008).

Neben dieser Vielzahl von Untersuchungen zum Einfluss regionaler Determinanten auf die aggregierte Größe des Beschäftigungsvolumens, die zumindest erste Hinweise auf risiko- und chancen-fördernde Rahmenbedingungen für Erwerbsverläufe geben, existieren nur wenige Studien, die regionale Indikatoren direkt in Erklärungsmodelle für die individuelle Beschäftigungsstabilität und Erwerbsverläufe einbeziehen. Auf der Basis von Linked-Employer-Employee Daten des IAB bilden Grotheer et al. (2004) den Einfluss wirtschaftlicher Rahmenbedingungen auf die Beschäftigungsstabilität von Arbeitnehmern ab, die neu in einen Betrieb eingestiegen sind. Ihre Schätzungen zeigen, dass Produktions- bzw. Nachfrageschwankungen, aber auch die regionalen Arbeitslosenquoten in Ostdeutschland aufgrund einer höheren Kompromissbereitschaft der Beschäftigten in den strukturschwachen Regionen stabilisierend, in Westdeutschland hingegen destabilisierend wirken. Analog dazu führen die beiden Einflussfaktoren in Westdeutschland zu häufigeren, in Ostdeutschland aber zu selteneren Betriebswechsel sowie Übergängen in Arbeitslosigkeit. Boockmann und Steffes (2005) ermitteln ebenfalls mit Linked-Employer-Employee Daten unter Berücksichtigung von Arbeitslosenquoten des Vorjahres auf der Ebene der Bundes- 
länder ähnliche Ergebnisse. Während sie für Westdeutschland keinen eindeutigen Effekt der Arbeitslosenquote auf die Beschäftigungsstabilität feststellen können, wirkt sie in Ostdeutschland stabilisierend. Darüber hinaus steigt mit der Arbeitslosenquote in Westdeutschland die Wahrscheinlichkeit, nach einer Beschäftigungsbeendigung arbeitslos zu werden. Bei ostdeutschen Frauen verringern sich zwischenbetriebliche Wechsel. In einer weiteren Arbeit finden Boockmann und Steffes (2010) auf Bundeslandebene hingegen keinen signifikanten Einfluss der Vorjahresarbeitslosenquoten auf die Beschäftigungsstabilität, jedoch auf eine verminderte zwischenbetriebliche Wechselrate in Westdeutschland.

Eine vergleichswiese hohe Humankapitalausstattung in einer Region erweist sich in wirtschaftlichen Aufschwungsphasen für alle Qualifikationsgruppen als beschäftigungs- und aufstiegsförderlich. Geringqualifizierte haben in Phasen des konjunkturellen Abschwungs trotz hoher regionaler Humankapitalausstattung vergleichsweise unsichere Beschäftigungsperspektiven und ein höheres Abstiegsrisiko (Dütsch und Struck 2014, S. 119). Mit zunehmender regionaler Arbeitslosigkeit geht eine geringere Wahrscheinlichkeit von Betriebsaustritten einher bzw. ist die betriebliche Beschäftigungsstabilität höher (ebd., S. 116).

Die Bedeutung konjunktureller Schwünge für Mobilitätsprozesse auf dem Arbeitsmarkt kann mit Hilfe des Sortiermodells aufgezeigt werden. Es erklärt Fluktuationen auf dem Arbeitsmarkt und hierbei insbesondere die effiziente Reallokation von Arbeitnehmern zu Arbeitsplätzen (Hinz und Abraham 2008; Struck 2006). Gemäß diesem Modell mündet eine Stellensuche aus einem bestehenden Arbeitsverhältnis heraus nur dann in einen Arbeitsplatzwechsel, wenn damit ein im Vergleich zur aktuellen Situation höherer monetärer bzw. nicht-monetärer Nutzen verbunden ist und zusätzlich die aufgewendeten Suchkosten aufgewogen werden. Somit gibt dieses Modell Hinweise auf das Kündigungsverhalten von Arbeitnehmern im Konjunkturverlauf. Unter der Annahme, dass im konjunkturellen Aufschwung zahlreiche, hinsichtlich der Entlohnung attraktive Arbeitsplätze entstehen, wird die zwischenbetriebliche Mobilität aufgrund freiwilliger Arbeitsplatzwechsel steigen und die durchschnittliche Beschäftigungsstabilität entsprechend sinken. Hingegen werden in einer Rezession nur wenige attraktive Arbeitsstellen geschaffen bzw. Stellen abgebaut. Zudem sind kaum Spielräume für Lohnerhöhungen vorhanden. Damit sinkt der Anreiz für freiwillige Mobilität, während unfreiwillige Freisetzungen zunehmen. 
Einige Forschungsarbeiten zu Erwerbsverläufen bilden den Effekt konjunktureller Zyklen ab (Erlinghagen 2005; Giesecke und Heisig 2010; Hübler und Walter 2009; Struck et al. 2007). Makrostudien zur Beschäftigungsdynamik auf dem deutschen Arbeitsmarkt können zwar die Übergangsgründe nicht explizit abbilden, stützen jedoch das Ergebnis, dass Arbeitsplatzwechsel prozyklisch verlaufen (Fitzenberger und Garloff 2007; Schaffner 2011).

Der Forschungsüberblick zu arbeitsmarkttheoretischen und regionalökonomischen Ansätzen und ihren Befunden zu betrieblicher Beschäftigungsstabilität und Betriebsmobilität hat gezeigt, dass auf Basis der bisherigen Arbeiten zu Erwerbsverläufen von einem antizyklischen Entlassungsrisiko und einem prozyklischen Kündigungsverhalten auszugehen ist. Zudem gibt es Erkenntnisse zu betrieblichen, sektoralen und individuellen Ursachen betrieblicher Ein- und Austritte. Allerdings gibt es nur sehr wenige Studien und Evidenz zu gruppenspezifischen Mobilitätsmustern bei freiwilligen Betriebswechsel. Regionale Disparitäten hinsichtlich der wirtschaftlichen Leistungsfähigkeit werden vor allem in aggregatsstatistischen, regionalökonomischen Studien betrachtet. Bezüglich ihres Einflusses auf Erwerbsverläufe wurden sie bislang kaum systematisch untersucht (mit Ausnahme von Dütsch und Struck 2014).

Die bisher vorgestellten Befunde beziehen sich auf Betriebsmobilität im Allgemeinen unabhängig davon, ob es sich um regionale oder überregionale Betriebsmobilität handelt. Unbeantwortet bleibt dabei, inwieweit die Ursachen für Betriebswechsel zugleich auch wichtige Determinanten für überregionale Betriebswechsel sind bzw. welche weiteren Einflussfaktoren bei Erklärungen von überregionaler Betriebsmobilität und ihres Erfolges zu berücksichtigen sind. Der folgende Abschnitt fokussiert auf die Erkenntnisse zu überregionalen Mobilitätsprozessen.

\subsection{Befunde zum räumlichen Arbeitsmarktverhalten}

Mit einem überregionalen Betriebswechsel kann eine punktuell auftretende Verlagerungen des Wohnortes, d.h. in Abhängigkeit der räumlichen Entfernung residentielle Mobilität (kleinräumige Wohnortmobilität) oder Binnenwanderung (Wohnortverlagerungen über administrative Gebiete, wie Gemeinden bzw. über größere Distanzen hinweg) oder zirkuläre Mobilität (d.h. Pendelmobilität), die episodenhaften Charakter hat und von der Rückkehr zu einem festen Ausgangspunkt gekennzeichnet ist, einhergehen (Dorn 2018, S. 43f.; Kley 2016; Scheider et al. 2016, S. 507). Ein Großteil der Binnenmobilität in Deutschland ist kleinräumig, d.h. Wohnortwechsel zwischen 
Städten und dem jeweiligen Umland bzw. zwischen benachbarten Regionen (Sander 2014²), in der Regel ohne Arbeitsortwechsel. Dennoch sind gemäß der BIBB/BAuA-Erwerbstätigenbefragung 2011/2012 circa ein Viertel der Befragten schon einmal aus einem beruflichen Grund umgezogen (eigene Berechnungen).

Analysen zur Binnenmobilität auf der Ebene von Bundesländern (Alecke und Untiedt 2000; Ale cke et al. 2010; Schlömer 2004; Windzio und Huinink 2010) oder feingliedriger auf der Ebene von Kreisen (Sander 2014) zeigen, dass die Wohnortmobilität (in Deutschland) in den letzten Jahren sehr stark von den Folgen der deutsch-deutschen Vereinigung geprägt war. Nach dem Fall der Mauer kam es zu einer massiven Abwanderung aus Ostdeutschland. Unmittelbar nach der deutschen Einigung waren in Ostdeutschland sehr hohe Wanderungsverluste von mehr als 165.000 Personen im Jahr zu verzeichnen. Diese negativen Wanderungssalden gingen bis Mitte der 1990er Jahre auf ca. 30.000 Personen zurück, wobei einerseits immer noch viele Menschen aus dem Osten in den Westen gingen, zugleich aber auch sogenannte „Aufbauhelfer“ in Ostdeutschland arbeiteten. Seit 1997 bis Anfang der 2000er Jahre stieg der negative Wanderungssaldo erneut auf einen Wert von knapp 100.000 Menschen (in 2001) an. Nach der erneuten Zunahme gingen die Wanderungsverluste besonders seit 2008 dann deutlich zurück (Windzio und Huinink 2010, S. 10f.; Windzio 2013, S. 669). Die Zielräume erstreckten sich überwiegend auf Regionen im Süden und der Mitte Westdeutschlands und deutlich seltener auf Norddeutschland. In den ersten Jahren nach der Wiedervereinigung (bis etwa 1995) waren die Entscheidungen für Ost-West-Wanderung stark von der räumlichen Entfernung, d.h. günstigen Lagebeziehungen zur Westgrenze, bestimmt, während die lokale wirtschaftliche Leistungsfähigkeit sowie die damit einhergehende arbeitsmarktstrukturelle Situation der Zielregion in den darauffolgenden Jahren (ab ca. 1996) stärker in den Vordergrund rückte (Schlömer 2004, S. 106). Neben den anfänglich bestehenden, aus geografischer Sicht naheliegenden Wanderungszielen an der ehemaligen innerdeutschen Grenze (östliches Niedersachsen, Großraum Hamburg, Nordhessen oder Oberfranken) haben mit dem Großraum München, Stuttgart, Köln sowie der Rhein-Main-Region neue, wirtschaftlich leistungsfähige Ziele an Bedeutung gewonnen. Damit haben sich die Ost-West-Wanderungen hinsichtlich ihrer Wanderungsziele zunehmend an die bestehenden gesamtdeutschen Strukturen großräumiger Mobilität angeglichen (Sander 2014; Schlömer 2004).

\footnotetext{
${ }^{2}$ Die Datengrundlage bilden die German Internal Migration (GIM)-Daten für den Zeitraum 1995 bis 2010.
} 
Darüber hinaus ist ein Süd-Nord-Gefälle erkennbar, auch wenn das Ausmaß im Vergleich zur Ost-West-Migration geringer ist. So verliert Norddeutschland eine nicht unerhebliche Personenzahl an Süddeutschland (Buch 2006, S. 245). Hierbei sind Hochschulabsolventen aus Nord- und Ostdeutschland häufiger regional mobil als diejenigen aus Süddeutschland (Fabian und Minks 2008).

Studien zur überregionalen Betriebsmobilität zeigen übereinstimmend, dass die Wahrscheinlichkeit regional mobil zu sein, mit dem Alter, dem Geschlecht und dem beruflichen Bildungsniveau variiert. So sind Beschäftigte mit einem Hochschulabschluss, jüngere Altersgruppen sowie Männer häufiger regional mobil ${ }^{3}$ (Granato et al. 2009; Haas 2000; Ruppenthal 2010; Schneider et al. 2002a, 2002b, S. 431; Wagner 1989). Zudem ist festzustellen, dass die regionale Mobilitätsneigung positiv mit Höhe des individuellen Tagesentgelts korrespondiert (Reichelt und Abraham 2015; Windzio 2004b) und insbesondere bei Arbeitskräften mit einer vergleichsweise kurzen Betriebszugehörigkeitsdauer besonders hoch ist (Windzio 2004b).

Bezüglich der wirtschaftlichen Gesamtlage ist analog zu den Befunden zu zwischenbetrieblicher Mobilität generell (Fitzenberger und Garloff 2007; Schaffner 2011) ein prozyklischer Verlauf von überregionalen Arbeitsplatzwechseln festzustellen (Haas 2000). Hervorzuheben ist jedoch, dass im Falle konjunktureller Aufschwünge regionale Mobilität zeitlich verzögert zunimmt, während sich Konjunkturabfälle durch einen sofortigen Rückgang der räumlichen Mobilität bemerkbar machen (ebd., S. 3).

Raumstrukturelle Untersuchungen zum Einfluss regionaler Disparitäten auf aggregierte Größen, wie beispielsweise das Beschäftigungsvolumen, können mit Blick auf das erwerbsbedingte räumliche Mobilitätsverhalten zudem Hinweise zu potentiellen Push- und Pull-Faktoren der Regionen geben. Hinsichtlich des Einflusses der regionalen Arbeitsmarktlage ist die empirische Befundlage jedoch nicht eindeutig. Während Windzio (2004a; 2004b) auf Basis von Individualdaten einen mobilitätshemmenden Effekt bei zunehmender Arbeitslosigkeit in der Herkunftsregion konstatiert, kommen Untersuchungen zu Wanderbewegungen von Arbeitskräften zu dem Ergebnis, dass Regionen mit Strukturproblemen, die eine hohe Arbeitslosigkeit und ein vergleichsweise geringes Lohnniveau aufweisen, von Abwanderung und negativen Wanderungssalden gekennzeichnet sind

\footnotetext{
${ }^{3}$ Der geschlechtsspezifische Mobilitätsunterschied ist auf intervenierende Faktoren, wie das berufliche Qualifikationsniveau, den Erwerbsumfang, etc. zurückzuführen. Unter Kontrolle dieser Effekte finden sich kaum Differenzen in der Mobilitätsneigung zwischen den Geschlechtern (Ruppenthal 2010).
} 
(Granato et al. 2009). Zudem finden sich erste Hinweise auf qualifikationsselektive Wirkungen des regionalen Arbeitslosigkeitsniveaus (ebd.). Demnach ergeben sich für Hochqualifizierte trotz objektiv schlechter regionaler Arbeitsmarktlage keine negativen Wanderungssalden, für Geringqualifizierte hingegen schon. Auf Basis von Stromgrößen sind keine Aussagen zum Einfluss regionaler Arbeitsmarktsituation auf das individuelle Mobilitätsverhalten möglich. Jedoch können so bildungsgruppenselektive Wirkungsweisen regionaler Strukturmerkmale herausgestellt werden, die es dann auf der Individualebene zu überprüfen gilt. Ebenfalls qualifikationsselektive Effekte strukturschwacher regionaler Arbeitsmärkte ermittelt Hillmert (2006, S. 3398) anhand einer Kohortenbetrachtung, allerdings entgegen der zuvor genannten Befunde dahingehend, dass Hochqualifizierte Regionen mit hoher Arbeitslosigkeit verlassen, Geringqualifizierte hingegen nicht. Für periphere, strukturschwache Räume lassen sich Abwanderungen in Arbeitsmarktzentren feststellen (Seibert 2008) ${ }^{4}$. Bezüglich der regionalen Qualifikationsstruktur zeigt sich, dass insbesondere eine berufsfachlich qualifizierten bzw. (hoch)qualifizierten Beschäftigtenstruktur das Beschäftigungswachstum einer Region positiv beeinflusst (Blien und Wolf 2002; Farhauer und Granato 2006; Schlitte et al. 2010; Shapiro 2006; Südekum 2008). Diese Befunde legen nahe, dass ein hoher Anteil an qualifizierten Beschäftigten ein regionaler Prosperitätsfaktor zu sein scheint und davon eine Sogwirkung auf Arbeitskräfte ausgeht. Allerdings stellen Schlitte et al. (2010) auf Basis des IAB-Betriebspanels für Westdeutschland eine zunehmende Segregation nach Qualifikationen in den Raumordnungsregionen fest (siehe auch Gerlach et al. 2002), was darüber hinaus eine divergente Entwicklung hinsichtlich der Beschäftigung sowie der Löhne hervorruft. Segregationsfördernd sind jedoch insbesondere dichtbesiedelte Regionen und Regionen, in denen der Dienstleistungssektor eine große Bedeutung hat. Diese Segregationstendenzen hinsichtlich der Qualifikation auf dem regionalen Arbeitsmarkt verdeutlichen, dass regionale Prosperitätsfaktoren, wie ein hohes regionales Lohnniveau, Bruttoinlandsprodukt, etc. nicht auf alle Qualifikationsgruppen der betreffenden Region eine beschäftigungssichernde und aufstiegsförderliche Wirkung entfalten, sondern hinsichtlich der Einkommens- und Erwerbschancen qualifikationsselektive Effekte zu erwarten sind. Regionen mit hohen Akademikeranteil erweisen sich als mobilitätsförderlich auf der Ebene von Großregionen (Windzio 2004b, S. 42).

\footnotetext{
${ }^{4}$ Die Untersuchung bezieht lediglich auf die Region Berlin-Brandenburg.
} 
Richtet man den Blick auf Hochqualifizierte, so ist festzustellen, dass ein hohes lokales Preis/Lohnniveau die Zuwanderung dieser Qualifikationsgruppe begünstigt, während für eine vergleichsweise gute lokale Qualifikationsausstattung kein positiver Effekt auf die Zuwanderung von Hochqualifizierten nachgewiesen werden kann (Buch et al. 2014). Zudem verzeichnen Regionen mit vergleichsweise höheren Beschäftigungschancen einen steigenden Zustrom an (hoch-)qualifizierten Arbeitskräften (Arntz et al. 2014). Akademiker in städtischen Regionen sind weniger mobil als in ländlichen Räumen. Die Ursache hierfür wird in vergleichsweise guten Beschäftigungsmöglichkeiten für Hochqualifizierte in Agglomerationsräumen gesehen (Rolfes 1996).

Generell stellen Studien zu räumlichen Mobilitätsprozesse die Bedeutung von Distanzen heraus (Ravenstein 1972). Je geringer die zu überwindenden Entfernungen sind, desto häufiger kommt es zu Wanderungen (Windzio 2013, S. 668). Sind Zielräume mehr als 100 km entfernt, nimmt die Wanderhäufigkeit deutlich ab und stagniert auf diesem Niveau (Schlömer 2004, S. 97). Dies gilt auch für Migrationsbewegungen zwischen Nord- und Süddeutschland (Windzio 2004b, S. 41).

Der Überblick über die Studienergebnisse zu zwischenbetrieblicher und überregionaler Beschäftigungsmobilität auf der Individualebene und die Betrachtung der regionalökonomischen Forschungsbeiträge verdeutlichen: Erstens wurden in der Erwerbsverlaufsforschung regionale Bedingungen weitgehend vernachlässigt bzw. allenfalls anhand regionaler Arbeitslosenquoten abgebildet. Zweitens können die Ergebnisse der Regionalforschung aufgrund der fehlenden Mikrofundierung nicht im Sinne der Erwerbsverlaufsforschung interpretiert werden. Gleichwohl geben sie wichtige Hinweise für zu beachtende Einflussgrößen. Dies betrifft insbesondere die Siedlungsstruktur und -dichte bzw. den Agglomerationsgrad, die regionale wirtschaftliche Leistungsfähigkeit und die Humankapitalausstattung. Diese regionalen Determinanten werden in den vorliegenden Untersuchungsbeiträgen systematisch berücksichtigt. Drittens werden betriebliche Rahmenbedingungen (Kapitalausstattung, Betriebsgröße, etc.) im Zusammenhang mit überregionaler Betriebsmobilität kaum bzw. lediglich anhand von betrieblichen Rekrutierungsstrategien auf Basis von Betriebsdaten untersucht (Gerstenberg 2014). Die in diesem Rahmen aufgezeigten betrieblichen Rekrutierungsmuster legen nahe, dass die individuellen erwerbsbedingten Mobilitätschancen von betrieblichen Charakteristika strukturiert werden. In Individualanalysen zu räumlicher Betriebsmobilität blieben Betriebsmerkmale bislang meist unbeachtet. 
Weitestgehend unerforscht ist darüber hinaus, welche Beschäftigtengruppen unter welchen betrieblichen und regionalen Bedingungen im Zuge von regionaler und überregionaler Betriebsmobilität Einkommenszuwächse erzielen können. Die wenigen vorliegenden Befunde werden im Folgenden skizziert.

\subsection{Befunde zur Einkommensentwicklung im Zuge von Betriebsmobilität}

Mit Blick auf die Einkommensentwicklung im Zuge überbetrieblicher Mobilitätsprozesse steigen aus humankapitaltheoretischer Sicht im Zeitverlauf durch die Akkumulation jeweils betriebsspezifischer Kenntnisse und Qualifikationen die Produktivität der Arbeitskräfte sowie deren Entlohnung (Becker 1975). So können insbesondere jüngere Beschäftigte im Zuge von Betriebswechseln Lohnzuwächse realisieren (Fitzenberger und Garloff 2007). Aufstiegsfördernde Effekte regionaler Mobilität in den ersten fünf Jahren der Erwerbskarriere können Reichelt und Abraham (2015) bestätigen. Kratz und Brüderl (2013) stellen für in Westdeutschland lebende Männer ${ }^{5}$ positive Einkommenseffekte räumlicher Mobilitätsprozesse fest. Unter Kontrolle von selektiv wirkenden Individualmerkmalen werden Einkommenssteigerungen im Zuge regionaler Mobilität auf 2 bis 3 Prozent geschätzt (Lehmer und Möller 2008).

Für den Großteil der hochqualifizierten Beschäftigten lassen sich im Zuge regionaler Mobilität vertikale Aufstiege realisieren (Hillmert 2006, S. 3398; Seynstahl 2015, S. 142). Hochschulabsolventen profitieren insbesondere zu Beginn ihrer Erwerbskarriere von überregionaler Betriebsmobilität (Maier und Sprietsma 2016).

Hacket (2009) weist für die Gruppe der Berufsanfänger nach, dass in Westdeutschland regional mobile Beschäftigte der unteren Bildungsgruppe im Vergleich zu innerregional mobilen Beschäftigten sowohl höhere Verlustrisiken als auch höhere Gewinnchancen aufweisen. Bei Hochqualifizierten gehen mit regionaler Mobilität lediglich geringere Einkommensverlustrisiken einher. Für Betriebswechsel von West- nach Ostdeutschland ließen sich nur für Hochqualifizierte Einkommenserfolge nachweisen, wohingegen Beschäftigte ohne beruflichen Abschluss hohe Verlustrisiken haben. Bei einem Verlassen des Ausgangsarbeitsorts in Ostdeutschland in Richtung West-

\footnotetext{
${ }^{5}$ Betrachtet werden lediglich Männer, die zum ersten Erhebungszeitpunkt in Westdeutschland lebten. Ostdeutsche sind nicht Teil der Untersuchungsgruppe, da die Autoren argumentieren, dass Wanderungsbewegungen im Zuge der deutschen Einigung eigenen spezifischeren Mustern folgen.
} 
deutschland sind die Chancen, unabhängig vom Bildungsniveau Einkommensgewinne zu erzielen, höher und die Verlustrisiken geringer als bei regionalen Arbeitsplatzwechseln innerhalb Ostdeutschlands (ebd.). Anzumerken ist, dass die teilweise erheblichen Unterschiede in den Lebenshaltungskosten zwischen Ost- und Westdeutschland unberücksichtigt blieben.

Positive Einkommensentwicklungen weisen Glaeser und Maré (2001) nach, wenn Beschäftigte in Agglomerationsräume wechseln. In Metropolregionen, welchen aufgrund der hohen Konzentration des Arbeitsangebots und der Arbeitsnachfrage eine verbesserte Zuordnung von Arbeitskräften und Arbeitsplätzen zugeschrieben wird (Glaeser und Maré 2001; Wheeler 2006), liegen die Löhne räumlich mobiler Beschäftigter allerdings unmittelbar nach dem Wechsel unter dem Lohnniveau der in der jeweiligen Zielregion bereits erwerbstätigen Arbeitskräfte (ebd.). Lehmer und Möller (2008) beobachten positive Einkommensverläufe nach regionaler Mobilität unabhängig vom regionalen Zielkontext. Für ländliche Zielregionen konnten negative Einkommenseffekte nachgewiesen werden (Lehmer und Ludsteck 2011, S. 559). Insgesamt wird festgestellt, dass Einkommenssteigerungen umso größer ausfallen, je geringer die Bevölkerungskonzentration in der Ausgangsregion und je größer die Besiedlungsdichte in der Zielregion ist (ebd., S. 559).

Werden betriebliche Charakteristika, wie die Anzahl der Beschäftigten und Qualifikationsstruktur der Belegschaft, mit in die Analyse einbezogen, so zeigt sich, dass höhere Lohnzuwächse in urbanen Räumen größtenteils durch eine Beschäftigung in Großbetrieben mit einer ausgesprochen guten Qualifikationsausstattung erklärt werden können (Kelle 2016). Unter der Kontrolle betrieblicher Kontextmerkmale kann nur ein sehr geringer Teil der beobachteten positiven Einkommensentwicklungen in Agglomerationsräumen dem unterstellten effizienteren Zuordnungsprozess von Arbeitskräften zu Arbeitsplätzen in urbanen Räumen zugeschrieben werden (ebd.).

Gemäß internationalen Studien unterliegen neben der Beschäftigungsdynamik auch die Löhne prozyklischen Schwankungen. Demnach sinken die Löhne in Abschwungphasen sowohl für die bereits bestehende Belegschaft als auch - in etwas stärkerem Maße - für Betriebseinsteiger (Devereux und Hart 2006; Hart 2006).

Anhand der wenigen bisher existierenden Untersuchungsergebnisse wurde deutlich, dass neben individuellen Einflussfaktoren wie beispielsweise Alter, berufliche Qualifikation, Betriebszugehörigkeit, auch regionale und betriebliche Kontexte die Einkommenserfolgswahrscheinlichkeit von (überregionalen) Betriebswechseln beeinflussen. Hinsichtlich regionaler Kontexte wurden 
insbesondere Effekte der Agglomeration untersucht. Befunde zum Einfluss der regionalen Qualifikationsstruktur und der Wirtschaftskraft liegen auf der Individualebene nicht vor. Es fehlen darüber hinaus bundesweitgeltende, kohortenübergreifende Untersuchungsergebnisse, die Betriebsmerkmale sowie das regionale Preisniveau berücksichtigen.

\subsection{Zwischenfazit}

Insgesamt ist festzuhalten, dass die vorgestellten Untersuchungen wesentliche Prädiktoren der Betriebsmobilität herausstellen. Dennoch existieren kaum Studien, die das räumliche Arbeitsmarktverhalten und die damit einhergehenden Einkommenseffekte von Vollzeitbeschäftigten bundesweit, unabhängig von der Zugehörigkeit zu bestimmten Eintritts- oder Austrittskohorten oder deren Ausgangsregionen, in den Blick nehmen.

Trotz der teilweise räumlich begrenzten Gültigkeit, des zum Teil fehlenden Individualbezugs und den oftmals deskriptiven Analyseformen geben die skizzierten Befunde gleichwohl erste wichtige Hinweise auf mögliche bildungsselektive Effekte der Einflussfaktoren von (überregionaler) Betriebsmobilität. Dies gilt insbesondere für die sich abzeichnende qualifikationsselektive Wirkung regionaler Kontextmerkmale. Darüber hinaus bleiben in den Mobilitätsanalysen, die sich auf Wechsel zwischen Großregionen beziehen, kleinräumigere wirtschaftsgeografische und arbeitsmarktstrukturelle Unterschiede, wie die regionale wirtschaftliche Leistungsfähigkeit und das regionale Preisniveau, unbeleuchtet. Deshalb bedarf es Analysen zu erwerbsbezogenen Mobilitätsprozessen, die feingliedrigere räumliche Analyseraster zugrunde legen als die vorliegenden Großregionenbetrachtungen, die regionale Kontextmerkmale auf ebendieser räumlichen Analyseebene erfassen und zudem betriebliche Charakteristika berücksichtigen.

Hinsichtlich der Qualität von regionalen Mobilitätsprozessen liegt bislang nur eine geringe empirische Evidenz zu den Determinanten zu Einkommenszuwächsen und -einbußen vor. Zumal hierbei der betriebliche und regionale Kontext der Zielbetriebe bislang nicht systematisch berücksichtigt wird. Weitgehend unbekannt ist zudem, ob regionale Charakteristika der Region, in der sich der Zielbetrieb befindet, auf die verschiedenen Beschäftigtengruppen, insbesondere Qualifikationsgruppen selektiv wirken.

Um die aufgezeigten Forschungslücken zu schließen, wird (überregionale) Betriebsmobilität und deren Konsequenzen für das Erwerbseinkommen im folgenden Kapitel zunächst theoretisch beleuchtet. 


\section{Theoretisches Modell und methodische Umsetzung}

\subsection{Theoretisches Modell: Entscheidung zu (überregionaler) Betriebsmobilität}

Wie allgemein in der Soziologie geht es auch in dieser Arbeit um die Frage, in welchem Verhältnis stehen individuelle Merkmale zu gesellschaftlichen Strukturen. Diese Fragestellung lässt sich nur jeweils bezogen auf den konkreten Gegenstand - hier überbetriebliche und überregionale Mobilitätsprozesse - beantworten (Coleman 1991).

Die Entscheidung, den Arbeitgeber innerregional oder überregional zu wechseln, wird meist mikrotheoretisch mittels eines nutzenorientierten Handlungsmodells abgebildet (Friedman und Hechter 1988). Dabei resultiert die Entscheidung, eine (Vollzeit-)Beschäftigung zugunsten einer anderen (Vollzeit-)Beschäftigung innerhalb der (Raumordnungs-)Region oder über die Grenzen der (Raumordnungs-)Region hinausgehend aufzugeben, aus dem Vergleich der unmittelbaren betrieblichen und lokalen Gegebenheiten mit alternativen Beschäftigungsangeboten und deren Rahmenbedingungen vor dem Hintergrund der individuellen, insbesondere arbeitsmarktrelevanten Ressourcen und Restriktionen des Beschäftigten.

Theoretische Modelle zu Wohnortmobilität gehen bei Mobilitätsentscheidungen im Lebenslauf von mehrstufigen Entscheidungsprozessen aus (Kalter 1997; Kley 2009; Schneider et al. 2002a). Mobilitätsgedanken können sich, wenn die individuellen Ressourcen als vorhanden angesehen werden und private und berufliche Ziele dadurch realisierbar werden oder bleiben, in Mobilitätspläne konkretisieren und schließlich zur Entscheidung zu regionaler oder überregionaler Betriebsmobilität führen (De Jong und Fawcett 1981; Kalter 1997).

Beschäftigte bewerten demnach die unmittelbaren betrieblichen und lokalen Gegebenheiten sowie alternative Arbeitsangebote und deren Kontextbedingungen vor dem Hintergrund ihrer individuellen Ressourcen, Ziele und Restriktionen und treffen auf dieser Basis eine Entscheidung den Betrieb innerhalb der Region oder überregional zu wechseln. Individuelle Ressourcen und Hindernisse hängen von der beruflichen und privaten Lebenssituation sowie den beruflichen und privaten Lebenszielen und dem ortgebundenen Kapital ab.

Auf Basis von humankapitaltheoretischen Überlegungen (Becker 1962; Mincer 1958) kann erwerbsbedingte regionale Mobilität als Zusatzinvestition zur Verbesserung der Verwertung von Humankapital und damit verbundenen höheren Erwerbschancen begriffen werden (Sjaastad 1962, 


\section{Theoretisches Modell und methodische Umsetzung}

S. 83). Die Wahrscheinlichkeit betrieblicher Mobilität steigt, wenn von einem intra- oder interregionalen Arbeitsplatzwechsel langfristig, d.h. unter Berücksichtigung der Aspekte des privaten Kontextes, der Lebensqualität, der Pendel- oder Umzugskosten etc., ein höherer Gesamtnutzen erwartet wird als bei einem Verbleib beim derzeitigen Arbeitgeber bzw. in der Region, in der der aktuelle Arbeitsort liegt. Mit Blick auf das Erwerbseinkommen sollte dann gelten, dass überregional mobile Arbeitskräfte im Vergleich zu nicht mobilen Beschäftigten häufiger Einkommenszuwächse realisieren können.

Humankapitaltheoretischen Überlegungen zufolge hängt die Entscheidung für einen Arbeitgeberwechsel stark von dem Interesse ab, die individuellen Bildungsinvestitionen bestmöglich verwerten zu können. Korrespondierend damit können qualifikationsspezifische Unterschiede hinsichtlich der räumlichen Mobilitätsneigung aus der Perspektive der Suchtheorie dadurch erklärt werden, dass die oftmals sehr spezifischen Qualifikationsanforderungen im Tätigkeitsbereich der Hochqualifizierten teilweise nur in spezialisierten überregionalen Arbeitsmärkten nachgefragt werden (Damelang 2007, S. 7), während sehr geringe Qualifikationsanforderungen fast flächendeckend unabhängig von siedlungs- und wirtschaftsstrukturellen Charakteristika Verwendung finden können (Meusburger 1998, S. 378). Beschäftigte mit höheren Qualifikationen und spezifischen Kompetenzprofilen, die ihren Arbeitsplatz wechseln möchten, haben auf dem überregionalen Arbeitsmarkt vergleichsweise bessere Chancen einen qualifikationsadäquaten Arbeitsplatz mit aussichtsreichen Einkommens- und Karriereperspektiven zu finden (Mertens und Haas 2006).

Kosten und Nutzen von überregionalen Arbeitsplatzwechseln sind zudem eng mit individuellen Wünschen, privaten und beruflichen Zielen verbunden, die wiederum vom familialen und sozialen Umfeld, bisherigen (Mobilitäts-)Erfahrungen, ortsabhängigen Ressourcen sowie der Stellung im Lebenszyklus beeinflusst werden. Aus lebensverlaufstheoretischer Perspektive treten gerade im frühen Erwachsenenalter verstärkt Wendepunkte im Lebenslauf auf. Solche „Statuspassagen“ (Heinz 1996) sind etwa der Erwerbseintritt, das Zusammenziehen mit einer Partnerin bzw. einem Partner oder eine Familiengründung etc. In diesen Orientierungs- und Entscheidungsphasen sind Beschäftigte in der Regel noch mobiler bzw. mobilitätsaffiner (Haas 2000, S. 6; Kley und Mulder 2010, S. 85; allg. auch Boyle et al. 1998). Mit zunehmendem Alter sind berufliche und private Wahl- und Entscheidungsprozesse, wie beispielsweise die Arbeitgeber-, die Wohnort- sowie die Partnerwahl, aber auch Heirat und Kinderplanung, weitgehend verwirklicht und demnach die soziale Einbettung in einer Region und eventuell auch in einem Betrieb weitgehend vollzogen. Aus 


\section{Theoretisches Modell und methodische Umsetzung}

diesem Grund ist davon auszugehen, dass die privaten Kosten, die aufgrund der räumlichen Trennung vom Haushaltskontext und vom sozialen Umfeld oder durch einen Haushaltsumzug mit erwerbsbedingter räumlicher Mobilität einhergingen, im Altersverlauf bis ins mittlere Erwerbsalter tendenziell steigen. Sie bleiben dann höher als in jüngeren Jahren und der relative Nutzen überbetrieblicher Mobilität sinkt. Dieser Effekt wird durch die in vielen Beschäftigungsbeziehungen vorherrschende Seniöritätsentlohnung (Lazear 1981) verstärkt. Demnach werden Arbeitskräfte zu Beginn ihrer Beschäftigung im Betrieb zunächst unterhalb der eigentlichen Produktivität entlohnt, wobei der Lohn mit zunehmender Betriebszugehörigkeit steigt. Mit Blick auf Arbeitsplatzwechsel ist folglich zu erwarten, dass die potentiellen Renditen von Arbeitgeberwechseln mit zunehmender Betriebszugehörigkeit bei Kontrolle des Alters abnehmen.

Zudem ist davon auszugehen, dass betriebliche Kontextfaktoren individuelle Mobilitätsentscheidungen formen (Esser 1996). Wie der Forschungsstand zeigt, können Charakteristika, wie beispielsweise das Lohnniveau, Prosperitätsfaktoren, wie die betrieblich antizipierte Personalentwicklung und die Entwicklung des Geschäftsvolumens etc. die betriebliche Beschäftigungsstabilität beeinflussen (u.a. Dütsch und Struck 2014). Die Zufriedenheit mit den Arbeitsbedingungen, dem Einkommen, den beruflichen Weiterentwicklungsmöglichkeiten und Einkommensperspektiven können die Neigung, in diesem Betrieb beschäftigt zu bleiben, erhöhen. Besteht jedoch eine Unzufriedenheit in Bezug auf die betrieblichen Beschäftigungsbedingungen, so ist zu erwarten, dass nach beruflichen Alternativen gesucht wird. So werden die Bedingungen des Ausgangsbetriebes mit möglichen Beschäftigungsoptionen außerhalb des Beschäftigungsbetriebs vor dem Hintergrund der individuellen beruflichen und privaten Situation, den Ressourcen, der beruflichen Karriereorientierung verglichen. Ob erwerbsbedingte Mobilität intraregional oder interregional erfolgt, hängt dann von den lokalen Beschäftigungsoptionen bzw. der individuellen Bewertung der regionalen Kontextmerkmale ab. Die jeweiligen betrieblichen Beschäftigungsbedingungen müssen nicht mit den lokalen Arbeitsmarktgegebenheiten korrespondieren. Gleichwohl können soziale und wirtschaftliche Strukturen auf der regionalen Ebene Erwerbs- und Lebenschancen beeinflussen. Deshalb sind neben individuellen Selektionsmechanismen und den Betriebsmerkmalen auch die regionalen wirtschafts- und siedlungsstrukturellen Gegebenheiten zu berücksichtigen (Schneider et al. 2002a, S. 37). 


\section{Theoretisches Modell und methodische Umsetzung}

Regionale Kontextbedingungen werden von Beschäftigten bewusst wahrgenommen und in ihrem Karriereverhalten berücksichtigt. Darüber hinaus können sie jedoch auch unbemerkt als strukturelle Restriktionen oder Ressourcen indirekt Erwerbsverläufe beeinflussen (Coleman 1990; Esser 1996). Migrationstheoretische und regionalökonomische Ansätze geben hierbei wichtige Hinweise. Strukturindikatoren wie die Bevölkerungsdichte, das Lohnniveau sowie das Ausmaß an Wirtschaftskraft und Arbeitslosigkeit sind räumlich ungleich verteilt und gelten als Push- oder Pull-Faktoren (Sjaastad 1962). Zentrale regionale Charakteristika, wie Arbeitslosigkeit oder wirtschaftliche Prosperität werden von den Erwerbspersonen subjektiv wahrgenommen und beurteilt (Lee 1972; Wolpert 1965). Integriert man diese migrationstheoretischen und regionalökonomischen Überlegungen in das Handlungsmodell, dann resultiert die Entscheidung, den Arbeitsplatz interregional zu wechseln, aus dem Vergleich der regionalen Gegebenheiten zwischen Ziel- und Herkunftsregion. Gemäß dem place utility-Ansatz (Wolpert 1965, S. 161ff.) messen Beschäftigte jedem potenziellen Arbeitsort einen gewissen Nutzenwert (place utility) bei. Von Bedeutung sind hierbei die mit einer möglichen regionalen Mobilitätsentscheidung einhergehenden beruflichen, persönlichen und familiären Folgen. Der individuellen Attraktivitätsbewertung eines Ortes steht das individuelle Anspruchsniveau (aspiration level) gegenüber, welches Nutzenansprüche bezüglich der gleichen Aspekte umfasst. Räumliche Mobilität wird dann zur Handlungsoption, wenn das Anspruchsniveau die Nutzenschwelle des bisherigen Arbeitsortes übersteigt und/oder die Nutzenschwelle eines alternativen Arbeitsortes die des bisherigen Arbeitsortes überschreitet. Da das subjektive Anspruchsniveau und die Bewertung regionaler Bedingungen stark von individuellen Merkmalen abhängen, werden in den nachfolgenden Analysen mögliche alter- und bildungsselektive Effekte von regionalen Kontextfaktoren mitberücksichtigt.

Die individualistische nutzentheoretische Perspektive lässt sich mit regionalökonomischen und raumstrukturellen Forschungsansätzen verbinden. Gemäß dem Zentrum-Peripherie-Modell (Krugman 1991) nutzen Betriebe und Arbeitskräfte in urbanen Räumen Agglomerationsvorteile aus. Danach versucht ein Betrieb um hohe Skalenerträge zu erzielen, die Produktion auf wenige oder einen Standort zu beschränken und von dort aus den Markt zu bedienen. Um Transportkosten zu vermeiden, siedeln sich Betriebe in Regionen mit großer Nachfrage, d.h. einer hohen Bevölkerungsdichte, an. Dadurch wiederum werden Arbeitskräfte und weitere Betriebe attrahiert. Letztere realisieren Agglomerationsvorteile infolge eines großen Absatzmarktes oder eines großen Arbeitskräftepools in einer solchen Region (ebd.). Regionalökonomische Forschungen können 


\section{Theoretisches Modell und methodische Umsetzung}

eine stärkere Arbeitskräftenachfrage und somit vergleichsweise gute Beschäftigungschancen in dicht besiedelten Regionen bestätigen (Blien et al. 2006; Farhauer und Granato 2006). In ländlichen Regionen ist die Dichte der potentiellen (adäquaten) innerregionalen Jobalternativen geringer als in dichter bevölkerten Räumen, die aufgrund der höheren Konzentration von Unternehmen zahlreiche Beschäftigungsmöglichkeiten bieten (Fassmann und Meusburger 1997, S. 223). Dabei sind Agglomerationsräume attraktive Zielregionen für zunächst alle Beschäftigtengruppen. Das in zentralen Räumen breitere Arbeitsplatzangebot sowie die günstigen Arbeitsmarktbedingungen erhöhen zum einen die Beschäftigungschancen der wechselaffinen Person selbst und kann - sofern es zu einem Haushaltumzug kommt - auch die qualifikationsadäquate Arbeitsplatzsuche der Lebenspartnerin bzw. des Lebenspartners erleichtern (Nisic 2011, S. 157). Dadurch, dass Großstädte im Vergleich zu weniger dicht besiedelten Regionen einen deutlich überdurchschnittlichen Anteil an hochqualifizierten Beschäftigten (Statistisches Bundesamt 2016, S. 357) und damit an Arbeitsplätzen für (fach-)hochschulisch Qualifizierte aufweisen, ist von einer Sogwirkung von Agglomerationsräumen vor allem bei hochschulisch Qualifizierten auszugehen. Es wird daher angenommen, dass die regionale Siedlungsstruktur bzw. Agglomerationsvorteile qualifikationsselektiv auf die Mobilitätsneigung wirkt.

Gemäß dem dualen Raumkonzept nach Fassmann und Meusburger (1997) bildet sich dann ausgehend von einer stabilen Güternachfrage und der Ansiedlung leistungsfähiger Unternehmen ein primäres Arbeitsmarktsegment in zentralen Räumen aus, das durch stabile Arbeitsplätze, gute Bezahlung, Aufstiegschancen sowie vorwiegend gut qualifizierte Arbeitskräfte gekennzeichnet ist. In peripheren Räumen entwickelt sich hingegen aufgrund der instabilen Nachfrage und weniger leistungsstarker Unternehmen ein sekundäres Arbeitsmarktsegment mit instabileren und geringer bezahlten Arbeitsverhältnissen, geringeren Qualifikationsanforderungen und weniger Aufstiegschancen. Demzufolge dürften die arbeitsmarktstrukturellen Bedingungen in wirtschaftlich starken, zentralen Lagen die Einkommensentwicklung positiv beeinflussen.

Dass regional divergierende Arbeitsmarktbedingungen Einfluss auf das räumliche Arbeitsmarktverhalten nehmen, konnte empirisch bestätigt werden (Hillmert 2006; Windzio 2004a, 2004b). Allerdings werden dabei hinsichtlich der Wirkungsrichtung unterschiedliche, zum Teil widersprüchliche Ergebnisse erzielt, die andeuten, dass etwa die regionale Arbeitslosenquote selektiv, insbesondere nach Alter und Qualifikation auf die Mobilitätsneigung von Beschäftigten, wirkt. Da mit einem zunehmenden Alter von Personen deren berufliche und soziale Bindung an eine 


\section{Theoretisches Modell und methodische Umsetzung}

Region und damit die Transaktionskosten bei Arbeitsortwechseln steigen, sollten die Ambitionen der älteren Beschäftigten, den Arbeitsort zu wechseln, auch bei einer ungünstigen regionalen Arbeitsmarktlage vergleichsweise geringer sein. Jüngere Beschäftigte weisen entsprechend dem Job Shopping-Ansatz (Johnson 1978) eine hohe Arbeitsplatzmobilität auf, um verschiedene Beschäftigungsoptionen auszutesten. In dieser beruflichen Orientierungsphase dürfen insbesondere Arbeitsplatzwechsel aus strukturschwachen in prosperierende Regionen vorgenommen werden.

In vergleichbare Richtung wie die regionale Arbeitslosigkeit sollten weitere wirtschaftliche Regionalindikatoren wirken. Die Höhe des Bruttoinlandprodukt und die Höhe des durchschnittlichen Arbeitnehmerentgelts in einer Region dürften als Pull-Faktoren fungieren und die Neigung, den Arbeitsplatz in solchen Regionen zu haben oder in solche Regionen zu verlegen, erhöhen, da hiermit die grundsätzliche Möglichkeit, Beschäftigungs- und Einkommensperspektiven verbessern zu können, erhöht ist. Anders als bei der regionalen Arbeitslosigkeit, bei der ich bezogen auf die überregionale Mobilitätswahrscheinlichkeit annehme, dass sie unterschiedlich auf verschiedene Qualifikations- und Altersgruppen wirken kann, sollten die letztgenannten regionalwirtschaftlichen Kontextbedingungen gruppenunspezifisch wirken und bei Zuwanderung in eine wirtschaftlich leistungsstarke Region insgesamt aufstiegsförderlich sein.

Die sogenannte endogene Wachstumstheorie der Regionalforschung stellt $u$. a. mit Verweis auf Annahmen von Humankapitalansätzen einen Zusammenhang zwischen der regionalen Qualifikationsstruktur der Beschäftigten und dem Wachstum sowie dem Wachstumspotential einer Region her (Lucas 1988; Romer 1986). Demnach führt eine steigende regionale Humankapitalausstattung zu einer steigenden Produktivität der Beschäftigten, sodass eine Konzentration von Humankapital einen „Wachstumsmotor“ für eine Region auch bei konstanter Technologie darstellt. Forschungsarbeiten können den positiven Einfluss der (hoch)qualifizierten Beschäftigtenstruktur auf das Beschäftigungswachstum einer Region bestätigen (Blien und Wolf 2002; Farhauer und Granato 2006; Schlitte et al. 2010; Shapiro 2006; Südekum 2008). Bezüglich der regionalen Qualifikationsstruktur ist zu erwarten, dass mit einer steigenden regionalen Humankapitalkonzentration die lokalen Beschäftigungsoptionen generell zunehmen.

Vertreter der Ansätze zum „skill-biased technological and organisational change“ (Acemoglu 2002; Bresnahan et al. 2002) machen zusätzlich darauf aufmerksam, dass infolge des technologischen und organisationalen Wandels die Beschäftigten in stärkerem Maße entlang des Merkmals der Qualifikation im Produktionsprozess getrennt eingesetzt werden und durch zielgerichtete 


\section{Theoretisches Modell und methodische Umsetzung}

Kombinationen von Qualifikationen im betrieblichen Arbeitsprozess die Produktivität der hochqualifizierten Arbeitskräfte noch steigt (ebd.). Folglich finden in Abhängigkeit von der jeweils bestehenden regionalen Qualifikationsstruktur Segregationsprozesse statt, so dass sich die Löhne und die Beschäftigungsperspektiven zwischen hoch- sowie geringqualifizierten Beschäftigten weiter auseinanderentwickeln. Demnach sind in Regionen mit einer hohen Humankapitalakkumulation qualifikationsselektive Einkommens- und Erwerbschancen zu erwarten.

Verbindet man die Argumentation einer qualifikationsbasierten Segregation auf dem lokalen Arbeitsmarkt (Acemoglu 1999; Duranton 2004) mit dem Ansatz der statistischen Diskriminierung (Arrow 1971; Phelps 1972), so ist darüber hinaus davon auszugehen, dass Betriebe in Regionen mit einer vergleichsweise hohen Humankapitalakkumulation auf ein großes Arbeitsangebot an qualifizierten Arbeitskräften zurückgreifen können. Demnach ist zu erwarten, dass sie bei ihrer Personalauswahl anspruchsvoller sein können, das heißt, sich nicht für Bewerber entscheiden werden, denen sie aufgrund von beobachtbaren, askriptiven Merkmalen eine geringere Produktivität zuschreiben. Frauen, Migranten und Personen, die längere Phasen der Arbeitslosigkeit aufweisen werden oftmals als weniger produktiv eingeschätzt (Arrow 1971; Arulampalam 2001; Evertsson und Grunow 2012; Phelps 1972; Spence 1973, 2002). Diese Gruppen könnten insbesondere in Regionen mit einer hohen Qualifikationsausstattung von Arbeitsgebern statistisch diskriminiert werden und dadurch höhere Erwerbsrisiken und schlechte Einkommenschancen aufweisen.

Neben den regionalen Strukturindikatoren sind aber auch ortsgebundene Ressourcen, mögliche Unterstützungsleistungen durch das familiale und soziale Umfeld in Bezug auf Versorgungs- und Betreuungsangelegenheiten sowie ortsgebundenes Kapital, wie beispielsweise Wohneigentum, als entscheidungsrelevante Faktoren zu berücksichtigen (Schneider et al. 2002a, S. 37).

Zudem sind im Modell gesamtwirtschaftliche Einflüsse zu kontrollieren. Dazu zählt, dass Mobilitätsprozesse im Konjunkturverlauf variieren. Unter der Annahme, dass im konjunkturellen Aufschwung bei steigendem Arbeitsangebot zahlreiche, hinsichtlich der Entlohnung attraktive Arbeitsplätze entstehen, sollte sowohl die zwischenbetriebliche als auch die interregionale Mobilität steigen. Hingegen werden in einer Rezession nur wenige attraktive Arbeitsstellen geschaffen bzw. Arbeitsplätze abgebaut. Zudem sind kaum Spielräume für Lohnerhöhungen vorhanden. Damit sinkt der Anreiz für freiwillige räumliche Mobilität, während unfreiwillige Freisetzungen zuneh- 


\section{Theoretisches Modell und methodische Umsetzung}

men (Giesecke und Heisig 2010; Struck et al. 2007). Anzunehmen ist daher, dass die erwerbsbedingte regionale Mobilität prozyklisch verläuft, da im konjunkturellen Aufschwung verstärkt überregionale Beschäftigungsangebote mit attraktiven Einkommensanreizen entstehen, die regionale Mobilitätsprozesse fördern. In gesamtwirtschaftlichen Abschwungphasen sinkt die Mobilität.

Vor dem Hintergrund regionaler Disparitäten gehe ich davon aus, dass Beschäftigte ökonomischrational agieren, indem sie betriebliche und regionale Kontexte in ihre Entscheidung den Betrieb zu wechseln, einbeziehen. Demnach sollten Erwerbstätige mit höherer Wahrscheinlichkeit betriebsmobil sein, wenn sie erwarten außerhalb des aktuellen Beschäftigungsbetriebs, ggf. durch einen überregionalen Wechsel, ein höheres Einkommen erhalten zu können.

\subsection{Methodische Umsetzung}

Die Datengrundlage für die Analyse überbetrieblicher und überregionaler Mobilitätsprozesse bilden Linked-Employer-Employee-Daten, konkret der integrierte Betriebs- und Personendatensatz (LIAB) des Instituts für Arbeitsmarkt- und Berufsforschung (IAB). Dieser verbindet die administrativen Arbeitnehmerdaten der Historikdatei der Beschäftigtenstatistik der Bundesagentur für Arbeit, die sehr valide Angaben zu Beschäftigungszeiten und den Löhnen enthalten, mit den Betriebsinformationen aus den jährlichen Befragungen des IAB-Betriebspanels (Jacobebbinghaus 2008). Diese Datenbasis wurde um ausgewählte regionale Strukturindikatoren auf der Ebene der Raumordnungsregionen im jeweiligen Wechseljahr ergänzt, welche der INKAR-Datensatz des Bundesinstituts für Bau-, Stadt- und Raumforschung (BBSR) bereitstellt.

Als räumlich abgrenzendes Analyseraster für den Arbeitsmarkt in Deutschland wird in Anlehnung an Rendtel und Schwarze (1996) das Konzept der Raumordnungsregionen herangezogen. Dieses umfasst 96 Raumordnungsregionen ${ }^{6}$, welche auf der räumlichen Einheit der Kreise basieren und in der Regel trennscharf mit den Grenzen der Bundesländer sind ${ }^{7}$. Die funktionalräumli-

\footnotetext{
${ }^{6}$ Die Anzahl der Raumordnungsregionen verringerte sich nach Kreisreformen in Sachsen (2008) und Sachsen-Anhalt (2007) von 97 auf nun 96 Raumordnungsregionen. Nachfolgende Kreisreformen, wie beispielweise in MecklenburgVorpommern 2011, wirkten sich nicht auf die Zahl der Raumordnungsregionen aus (BBSR 2016). Der Gebietsstand von 2008 wird für den gesamten Analysezeitraum (2000 bis 2010) angenommen.

${ }^{7}$ Eine Ausnahme bildet hier die Raumordnungsregion 13 „Bremerhaven“, welche neben der Stadt Bremerhaven (Freie Hansestadt Bremen) auch die niedersächsischen Landkreise Cuxhaven und Wesermarsch umfasst.
} 


\section{Theoretisches Modell und methodische Umsetzung}

che Abgrenzung mittels Raumordnungsregionen berücksichtigt sowohl infra- und erwerbsstrukturelle Disparitäten als auch Pendelbeziehungen zwischen dem Umland und den Oberzentren ${ }^{8}$. Demzufolge können Raumordnungsregionen als zusammengehörige funktionale Einheiten charakterisiert werden und stellen somit adäquate Abbildungen regionaler Arbeitsmärkte dar (Rendtel und Schwarze 1996).

In den Fachartikeln wird überregionale Betriebsmobilität als ein Arbeitgeberwechsel, der über die Grenzen des jeweiligen räumlichen Analyserasters (in diesem Fall eine Raumordnungsregion oder Großregionen) hinausgeht, operationalisiert - unabhängig davon, ob der Wechsel mit Pendeloder Wohnortmobilität einhergeht. Hierfür stellen die administrativen Beschäftigtendaten valide Informationen zu den Arbeitsorten der Beschäftigten auf der Ebene der Kreise bereit.

Betriebsmobilität wird als unmittelbarer Übergang von einer Vollzeitbeschäftigung in eine erneute Vollzeitbeschäftigung in einem anderen Betrieb analysiert ${ }^{9}$. Die Eingrenzung auf Vollzeitbeschäftigungen, die in eine erneute Vollzeitbeschäftigung münden, ist zum einen für den nächsten Schritt der Untersuchung - der Analyse von Einkommensveränderungen - wichtig, um die Vergleichbarkeit des Arbeitsumfanges ${ }^{10}$ sicherzustellen. Zum anderen ist die Beschränkung auf Vollzeitbeschäftigte aber auch ein Versuch die Mobilitätsmuster und Einkommenschancen in Bezug auf Erwerbsorientierungen möglichst konstant zu halten. Unterstellt wird damit, dass bei (überregionaler) Betriebsmobilität von Vollzeitbeschäftigten ebendiese Erwerbsorientierung wichtig ist. Die Beschränkung auf Vollzeiterwerbstätige wird als notwendig erachtet, da etwa mitziehende Lebenspartner(innen) oder andere Familienangehörige, für die ggf. noch andere Motive bedeutsam sind und die beispielsweise in Teilzeit arbeiten, auf Basis der erwerbsfokussierten

\footnotetext{
${ }^{8}$ Raumordnungsregionen werden auf Grundlage der Planungsregionen der Länder gebildet. Als Schwellenwert für eine von den Planungsregionen abweichende Zuordnung der Kreise zu den Raumordnungsregionen wird ein Anteil von Pendlern aus der Region von 15\% der sozialversicherungspflichtig Beschäftigten verwendet (BBSR 2016). ${ }^{9}$ Kurze Phasen der Nicht-Erwerbstätigkeit von maximal 90 Tagen werden ausgeblendet, da davon auszugehen ist, dass die Beschäftigten den zukünftigen Arbeitgeber zu dem Zeitpunkt, zu dem die vorangehende betriebliche Beschäftigung endet, meist bereits kennen. Als Phasen der Arbeitslosigkeit werden demnach Episoden der Nicht-Erwerbstätigkeit mit einer Dauer von mehr als 90 Tagen angesehen. Übergänge von Vollzeitbeschäftigungen in NichtVollzeiterwerbstätigkeiten (d.h. Teilzeit oder geringfügige Beschäftigung) werden in Bezug auf die Einkommensentwicklung nicht betrachtet.

${ }^{10}$ Sofern das Einkommen in Analysen berücksichtigt wird, ist eine Beschränkung auf die Betrachtung von Vollzeitbeschäftigungen notwendig. Andernfalls wäre die Einkommenshöhe sehr stark durch unterschiedliche Arbeitszeitumfang beispielsweise in geringfügiger Beschäftigung oder Teilzeitarbeit beeinflusst. Die administrativen Daten stellen sehr valide Angaben zum Erwerbseinkommen in Form von Tageseinkommen bereit, Angaben zu Arbeitsstunden oder zu Stundenlöhnen fehlen allerdings.
} 


\section{Theoretisches Modell und methodische Umsetzung}

Datenlage, die keine Informationen zu Motiven oder zum Haushaltskontext bereitstellt, nicht identifiziert werden können.

Unmittelbare Einkommensveränderungen im Zuge betrieblicher Mobilität werden als Einkommensaufstiege, laterale Mobilität und Einkommensabstiege untersucht. Als Aufstiege werden Lohnzuwächse von mehr als 10\% bezeichnet, während bei einem Rückgang des Lohnes von mehr als 5\% von einem Abstieg ausgegangen wird. ${ }^{11}$ Einkommensstabilität - laterale Mobilität - liegt dann vor, wenn sich das Einkommen lediglich bis maximal 10\% erhöht bzw. um bis zu 5\% sinkt. Um nicht absolute, sondern reale Einkommenssituationen vergleichen zu können, werden die Einkommen bei überregionaler Betriebsmobilität auf Basis von Daten des BBSR (Kawka 2009) um das lokale Preisniveau der Herkunfts- und Zielarbeitsorte auf der Ebene der Kreise bzw. Kreisregionen ${ }^{12}$ bereinigt.

Die Analyseergebnisse, der Fachartikel, die im Folgenden vorgestellt werden, beziehen sich auf Vollzeitbeschäftigte im Alter von 18 bis 65 Jahren. ${ }^{13}$ Das Analysezeitfenster erstreckt sich vom Beginn des Jahres 2000 bis Ende des Jahres 2010.

\footnotetext{
${ }^{11}$ Der Definition von Auf- und Abstiegen liegen asymmetrische Grenzen zugrunde, da anzunehmen ist, dass Einkommensverlustaversion besteht (Novemsky und Kahneman 2005), und Einkommensverluste bereits in kleinerem Aus$\mathrm{ma} ß$ als Situationsverschlechterung empfunden werden, während geringfügige Einkommenszuwächse von den Beschäftigten eher als selbstverständlich und inflationskompensierend angesehen werden.

${ }^{12}$ Kreisfreie Städte unter 100.000 Einwohnern werden zusammen mit ihrem/n Umlandkreis(en) als Kreisregionen betrachtet. Kreise bzw. Kreisregionen stellen die Analyseebene des hier herangezogenen Datenmaterials dar und wurden gewählt, um regionale Preisunterschiede möglichst feingliedrig abbilden zu können. Raumordnungsregionen, welche die Messebene der regionalen Strukturindikatoren darstellen, sind hinsichtlich regionaler Lohndifferenzen zu heterogene Raumeinheiten (Kawka 2009).

${ }^{13}$ Episoden mit einem Einkommen, das jenseits der Beitragsbemessungsgrenze der gesetzlichen Rentenversicherung liegt und daher zensiert ist, sind nicht im Analysesample enthalten.
} 


\section{Darstellung und Interpretation der eigenen Erkenntnisse}

Im folgenden Abschnitt werden die eigenen empirischen Befunde, die aus den vier Fachartikeln hervorgegangen sind, dargestellt und mit Blick auf deren Erkenntnisbeiträge zum Stand der Forschung interpretiert. Zunächst wird jedoch die wirtschaftsgeografische Ausgangslage skizziert.

\subsection{Wirtschaftsgeografische Ausgangslage}

Regionale Strukturindikatoren - die INKAR-Daten des Bundesinstitutes für Bau-, Stadt- und Raumforschung (BBSR) - gemessen auf der Ebene von Raumordnungsregionen verdeutlichen, dass hinsichtlich der regionalen Arbeitsmarktlage, der wirtschaftlichen Leistungsfähigkeit (Abbildung 1), der Qualifikationsstruktur der sozialversicherungspflichtig Beschäftigten und dem durchschnittlichen Lohnniveau erhebliche Unterschiede zwischen den Raumordnungsregionen bestehen.

Abbildung 1: Regionale Disparitäten (beispielhafte Darstellung der regionalen Strukturindikatoren Arbeitslosenquote und wirtschaftliche Leistungsfähigkeit (BIP) auf der Ebene von Raumordnungsregionen im Jahr 2006)
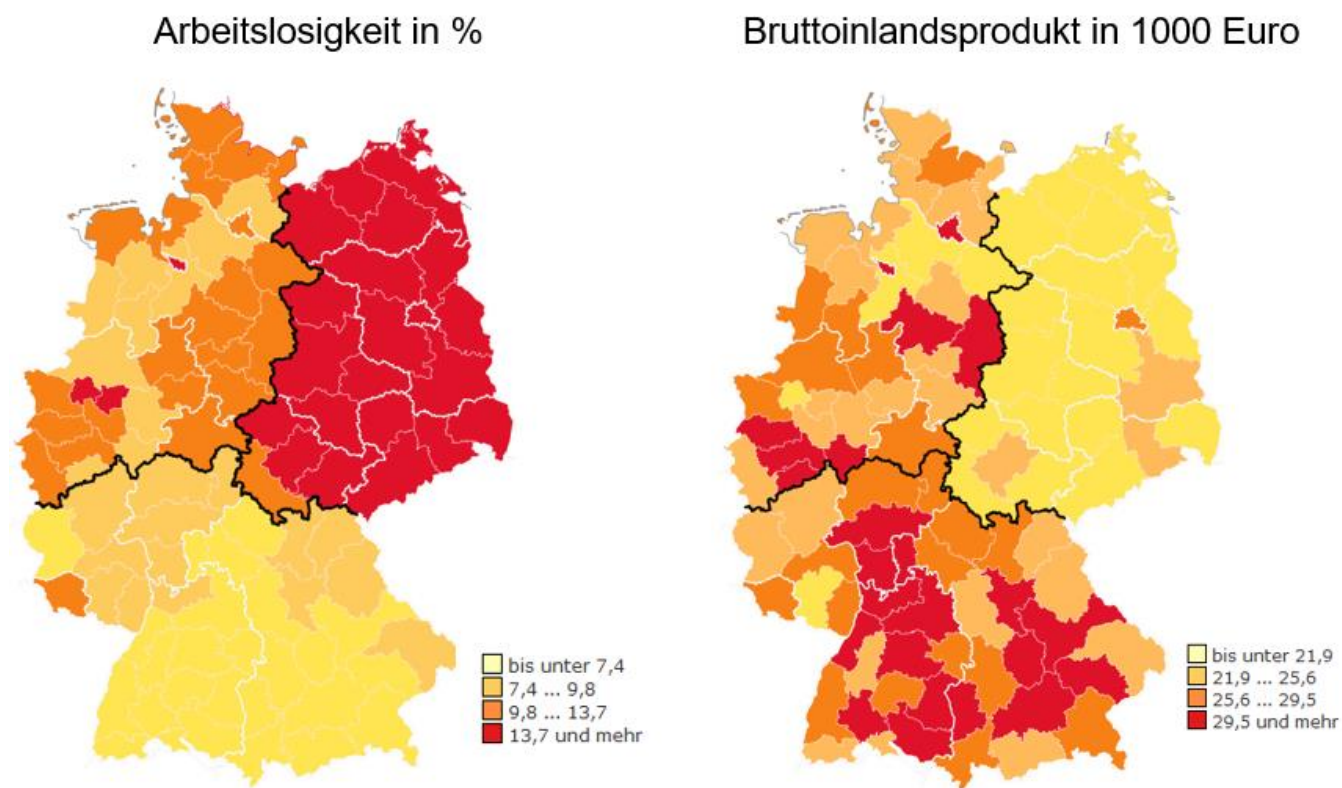

Die geografische Verortung dieser Niveauunterschiede lässt unschwer regionale Muster erkennen. So hebt sich der Großraum Süd hinsichtlich der Arbeitsmarktlage und der Wirtschaftskraft 


\section{Darstellung und Interpretation der eigenen Erkenntnisbeiträge}

positiv vom Bundesdurchschnitt ab, während Ostdeutschland deutlich ungünstigere Werte aufweist. Die Arbeitsmarktsituation und wirtschaftliche Leistungsfähigkeit in Norddeutschland entsprechen im Wesentlichen dem deutschlandweiten Durchschnitt. Siedlungsstrukturell ergibt sich für Deutschland folgendes Bild: 24 Agglomerationsräume, 35 Regionen mit Verstädterungstendenz und 37 ländlich geprägte Regionen. Der Osten Deutschlands ist stark ländlich geprägt, während in Norddeutschland (insbesondere in Nordrhein-Westfalen) urbane Strukturen dominieren. Den süddeutschen Raum kennzeichnen im Vergleich dazu sowohl periphere als auch (peri-)urbane Strukturen.

Vor dem Hintergrund der bestehenden regionalen Disparitäten gehe ich davon aus, dass Beschäftigte versuchen ökonomisch-rational zu agieren, indem sie regionale Einflussfaktoren in ihre arbeitsmarktrelevanten Entscheidungen einbeziehen. Demnach sollten Erwerbstätige mit höherer Wahrscheinlichkeit überbetrieblich und überregional mobil sein, wenn sie außerhalb des aktuellen Beschäftigungsbetriebs oder nach regionaler Mobilität höhere Einkommen erhalten können. Es ist eine empirisch offene Frage, welche der Faktoren, die im Stand der Forschung (Abschnitt 2) oder auf Basis der theoretischen Überlegungen (Abschnitt 3) als mögliche Einflussfaktoren vorgestellt wurden, sich als erfolgsfördernd erweisen. Bezieht man neben humankapitaltheoretischen Erklärungen (Becker 1975), Überlegungen von Segmentationsansätzen (Doeringer und Piore 1971; Sengenberger 1987) sowie das Zentrum-Peripherie-Modell (Krugman 1991), das duale Raumkonzept (Fassmann und Meusburger 1997), die endogene Wachstumstheorie (Lucas 1988; Romer 1986) und Gravitationsmodelle (Sjaastad 1962) mit ein, so ist davon auszugehen, dass Erwerbschancen und -risiken neben den Individualmerkmalen der Beschäftigten von betrieblichen Gelegenheitsstrukturen und raumstrukturellen Kontextfaktoren mitstrukturiert werden. Auf der regionalen Ebene geben die Ergebnisse der Regionalforschung trotz ihrer meist fehlenden Mikrofundierung Hinweise für relevante Einflussgrößen, die hinsichtlich ihrer Wirkungsweise als Push- und Pull-Faktoren (Lee 1972) bei Mobilitätsentscheidungen und damit in ihrer Bedeutung für Erwerbsverläufe zu überprüfen sind. Dabei sollten die regionalen Kontextbedingungen unterschiedlich auf die verschiedenen Alters- und Qualifikationsgruppen wirken. Unterstellt wird dabei, dass die beruflichen Karriereaspirationen, die Such- und Wanderradien (Mertens und Haas 2006) sowie die private Lebenssituation und Präferenzen, wie die räumliche Bindung an die Familie und das soziale Umfeld, zwischen den Beschäftigtengruppen differieren. Das Anspruchsniveau und die individuelle Bewertung der Kontextmerkmale (Place utility Konzept nach Wolpert 


\section{Darstellung und Interpretation der eigenen Erkenntnisbeiträge}

1965) erfolgt subjektiv und variiert mit der beruflichen und privaten Situation und den Lebenszielen sowie mit der Bindung an einen Ort. Da die Datengrundlage keine Informationen zu subjektiven Motiven u. ä. bereitstellt, konnten diese Aspekte nur näherungsweise über theoretisch gestützte Annahmen zu Kalkülen von Alters- und Qualifikationsgruppen einbezogen werden.

\subsection{Erkenntnisse aus dem Fachartikel „Employment trajectories in heterogeneous regi- ons: Evidence from Germany“}

Zunächst wurden vor diesem Hintergrund im Beitrag „Employment trajectories in heterogeneous regions: Evidence from Germany“ (Dütsch et al. 2019), der in der Zeitschrift Advances in Life Course Research publiziert wurde, die Determinanten zwischenbetrieblicher Mobilitätsprozesse untersucht. Der Analysefokus liegt hierbei insbesondere auf den Einfluss regionaler Merkmale, konkret auf dem Effekt von Agglomerationsräumen (Krugman 1991) und der damit einhergehenden Konzentration von Arbeitskräften innerhalb einer Raumeinheit (Wheeler 2006) und auf der regionalen Humankapitalausstattung, welcher gemäß der endogenen Wachstumstheorie eine Schlüsselrolle für das regionale Wachstumspotential (Lucas 1988; Romer 1990) zukommt. Zudem werden Betriebsmerkmale und arbeitsmarktbezogene Individualmerkmale berücksichtigt.

Die Ergebnisse der Cox-Schätzungen zeigen in Übereinstimmung mit bisherigen Befunden, dass individuelle und betriebsstrukturelle Charakteristika mit Blick auf Erwerbsverläufe bedeutsam sind (Boockmann und Steffes 2010; Dütsch und Struck 2014; Struck 2006). Frauen sind betriebsstabiler beschäftigt und haben im Falle eines Betriebsaustrittes ein geringeres Arbeitslosigkeitsrisiko als Männer. Die Tatsache, dass sie vergleichsweise selten zwischenbetriebliche Aufstiege realisieren oder Übergänge in Vollzeitbeschäftigungen vollziehen, spricht dafür, dass Frauen teilweise Kinderbetreuungsaufgaben übernehmen sowie häufiger in sozialen und pflegerischen Berufen und im öffentlichen Dienst tätig sind (Hausmann und Kleinert 2014; Hinz und Gartner 2005; Statistisches Bundesamt 2017, S. 32), die in der Regel durch vergleichsweise geringere Aufstiegsmöglichkeiten gekennzeichnet sind (ebd.; Wirtschafts- und Sozialwissenschaftliches Institut 2018).

Es zeichnen sich darüber hinaus Endogenitäten im Erwerbsverlauf ab (Mayer und Blossfeld 1990). So begünstigen berufliche Ausbildungsabschlüsse die betriebliche Beschäftigungsstabilität. Beruflich Qualifizierte und Akademiker haben seltener Einkommenseinbußen und wechseln seltener in geringfügige Beschäftigung oder in Arbeitslosigkeit. Hochqualifizierte realisieren im 


\section{Darstellung und Interpretation der eigenen Erkenntnisbeiträge}

Zuge von Betriebswechseln häufiger Einkommenserfolge. Zudem weisen insbesondere Personen, die anteilig längere Arbeitslosigkeitsphasen im bisherigen Erwerbsverlauf hatten, ein höheres Arbeitslosigkeitsrisiko auf.

Regionsspezifische Merkmale ${ }^{14}$ per se beeinflussen betriebliche Mobilitätsprozesse in geringem Maß. Generell ist die betriebliche Beschäftigungsstabilität in Agglomerationsräumen geringer. Jedoch sind in diesen Regionen die Beschäftigungsperspektiven aufgrund erhöhter Chancen auf direkte Betriebswechsel in sozialversicherungspflichtige Beschäftigungen und auch Einkommensaufstiege positiver als in ländlichen Regionen. Demnach können den Ergebnissen zufolge suchtheoretische Überlegungen, wonach die höhere Konzentration von Arbeitskräften in dicht besiedelten Regionen zu einer verbesserten qualifikatorischen und motivationalen Zuordnung von Arbeitskräften zu Arbeitsplätzen und damit zu einer höheren Beschäftigungsstabilität führt, nicht bestätigt werden (Wheeler 2006). Diese Überlegung scheint zu statistisch zu sein. Matchingprozesse können sich im Zeitverlauf ändern, da Tätigkeits- und Anforderungsprofile sowie die individuelle Qualifikationsausstattung nicht gleichbleiben müssen, sondern sich im Laufe der Zeit entwickeln. Zuordnungen von Arbeitskräften zu Arbeitsplätzen scheinen unter Berücksichtigung veränderter Ausgangsbedingungen gerade in Agglomerationsräumen, die vergleichsweise viele berufliche Alternativtätigkeiten bereitstellen, besonders gut und einkommenserfolgreich zu erfolgen. So kann gezeigt werden, dass Arbeitskräfte häufiger wechseln, wenn ein positives Marktumfeld besteht. Dabei sind Einkommenszugewinne in zentralen Lagen zu beobachten (so auch Glaeser und Maré 2001; Kelle 2016).

Mit der Humankapitalakkumulation einer Region erhöhen sich unseren Befunden zufolge die Erwerbsrisiken. Demnach lassen sich makrotheoretische Überlegungen, die davon ausgehen, dass Humanressourcen einen „Wachstumsmotor“ für Regionen darstellen, indem Arbeitsplätze geschaffen und die Nachfrage nach Arbeitskräften befördert werden (Glaeser 1999; Jovanovic und Rob 1989), und dahingehend bestehende empirische Beschreibungen der Regionalökonomie (Poelhekke 2013; Schlitte 2012; Shapiro 2006; Südekum 2008) nicht auf die Individualebene bzw. die individuellen Erwerbschancen übertragen. Dies ist überraschend: Möglicherweise ist die Marktkonkurrenz unter qualifizierten Beschäftigten in Regionen mit einem hohen Anteil an

\footnotetext{
${ }^{14}$ Betrachtet wurden regionale Charakteristika der Ausgangsregion. Von überregionalen Betriebswechseln wurde in diesem Aufsatz abstrahiert.
} 


\section{Darstellung und Interpretation der eigenen Erkenntnisbeiträge}

Hochqualifizierten vergleichsweise groß und - bei Beobachtung eines direkten Wechsels - zunächst chancenmindernd.

Mit Blick auf die Erwerbschancen unterschiedlicher Bildungsgruppen ergibt sich ein gemischtes Bild: So finden beruflich Qualifizierte in Räumen mit einer hohen Siedlungsdichte aussichtsreichere Erwerbsperspektiven vor als Gering- oder Hochqualifizierte. In Regionen mit einer vergleichsweise hohen Humankapitalausstattung findet eine Segregation nach Qualifikation zwischen Beschäftigten mit beruflichen und akademischen Abschlüssen statt. Frauen und Ausländer profitieren mit höherer Wahrscheinlichkeit von dicht besiedelten Räumen und einer vergleichsweise hohen Humankapitalkonzentration. Im Gegensatz dazu verschlechtern sich in diesen Regionen, die durch Zuwanderung und - so scheint es - durch höhere (Lohn-)Konkurrenz qualifizierterer Kräfte geprägt sind, die Erwerbschancen von Personen, deren bisherige Erwerbsbiografien von längeren Phasen der Arbeitslosigkeit geprägt waren. Lediglich für diese Beschäftigtengruppe können Überlegungen von Ansätzen der „skill segregation“ (Acemoglu 1999; Duranton 2004) in Verbindung mit Ansätzen zu statistischer Diskriminierung (Arrow 1971; Phelps, 1972) bestätigt werden. Für andere Personengruppen, denen häufig eine geringere Produktivität zugeschrieben wird, wie beispielsweise Frauen und Personen, die die keine deutsche Staatsbürgerschaft haben, (Arrow 1971; Arulampalam 2001; Evertsson und Grunow 2012; Phelps 1972), sind gegenteilige Effekte der Agglomeration und Humankapitalakkumulation festzustellen.

Daneben beeinflussen betriebliche Charakteristika die betriebliche Beschäftigungsstabilität und die Erwerbschancen. Die Existenz eines Betriebsrates, ein hohes Lohnniveau sowie ein betriebliches Ausbildungsengagement erhöhen die Wahrscheinlichkeit, in einem Betrieb beschäftigt zu bleiben. Erwarten Betriebe eine positive Personalentwicklung, so besteht für Betriebswechsler ein geringeres Arbeitslosigkeitsrisiko, während betriebliche Negativprognosen hinsichtlich der Entwicklung des Personals die Wahrscheinlichkeit von Betriebsaustritten und von Übergängen in Arbeitslosigkeit erhöhen. Höhere Arbeitslosigkeitsrisiken lassen sich auch beim Verlassen von Betrieben feststellen, die ein sinkendes Geschäftsvolumen erwarten. In diesen Fällen kommt es wohl zu unfreiwilligen Betriebsaustritten. Im Gegensatz dazu erhöhen steigende Erwartungen des Geschäftsvolumens die Wahrscheinlichkeit für Einkommensaufstiege in der Anschlussbeschäftigung. Vermutlich wird in diesen Fällen häufiger gezielt und freiwillig gewechselt. Im Vergleich zu dem produzierenden Sektor ist die betriebliche Beschäftigungsstabilität in allen übrigen Wirt- 
schaftszweigen geringer. Für den Bereich Bau sind zudem erhöhte Abstiegs- und Arbeitslosigkeitsrisiken festzustellen, im frauendominierten Dienstleistungsbereich sind Übergange in Teilzeit oder in geringfügige Beschäftigungen besonders wahrscheinlich.

Festzuhalten ist, dass betriebliche Beschäftigungsstabilität und überbetriebliche Mobilitätschancen neben Individualmerkmalen auch von betrieblichen und regionalen Bedingungen bestimmt werden. Für die regionalökonomischen Faktoren (Agglomeration und Humankapitalausstattung) und die damit einhergehenden Überlegungen lassen sich auf der Individualebene nur zum Teil die erwarteten Effekte nachweisen. Es ist allerdings eine offene Frage, ob und inwieweit die herausgestellten Einflussfaktoren auch bei der Untersuchung überregionaler Betriebsmobilität gelten. Bisherige Studien zu räumlicher Mobilität am Arbeitsmarkt besitzen diesbezüglich eine geringe Aussagekraft, da sie sich meist lediglich auf bestimmte Kohorten bzw. Beschäftigtengruppen oder Regionen beziehen. Darüber hinaus werden betriebliche und regionale Charakteristika weitgehend vernachlässigt. Deshalb wurden in den folgenden Beiträgen die Einfluss- und Erfolgsfaktoren, die überregionale Betriebsmobilität kennzeichnen, unter Berücksichtigung betrieblicher und regionaler Kontexte in den Blick genommen.

\subsection{Erkenntnisse aus dem Fachartikel „Regionale Mobilität am Arbeitsmarkt. Individu- elle, betriebliche und wirtschaftsstrukturelle Determinanten von Mobilität und Ein- kommen"}

Überregionale Mobilitätsprozesse wurden zunächst auf der Ebene von 96 Raumordnungsregionen im Beitrag „Regionale Mobilität am Arbeitsmarkt. Individuelle, betriebliche und wirtschaftsstrukturelle Determinanten von Mobilität und Einkommen“ (Ganesch et al. 2019), der in der Kölner Zeitschrift für Soziologie und Sozialpsychologie veröffentlicht wurde, analysiert. Hierzu wurden neben dem Agglomerationsgrad und der lokalen Humankapitalausstattung, die Arbeitsmarktlage, die wirtschaftliche Leistungsfähigkeit sowie das Lohnniveau der Raumordnungsregion berücksichtigt.

In Übereinstimmung mit dem empirischen Forschungsstand (Hillmert 2006; Windzio 2004a, 2004b) zeigte sich, dass die Wahrscheinlichkeit, überregional betriebsmobil zu sein, bei Berücksichtigung regionaler Strukturindikatoren überwiegend von Individualmerkmalen beeinflusst wird. Diese Grundaussage bestätigt bisherige nutzentheoretisch orientierte Befunde (Granato et al. 2009; Haas 2000). Gerade jüngere und qualifizierte Erwerbstätige versuchen über räumliche 


\section{Darstellung und Interpretation der eigenen Erkenntnisbeiträge}

Mobilität ihre Humankapitalinvestitionen erfolgreich zu verwerten. Sie wandern häufiger. Zudem wird mit Blick auf Einkommenszuwächse deutlich, dass sie mit größerer Wahrscheinlichkeit auch einkommenserfolgreich sind.

Bezüglich regionaler Strukturindikatoren geht mit zunehmender wirtschaftlicher Leistungsfähigkeit in der Ausgangsregion eine erhöhte Mobilitätsneigung einher. Strukturschwache und ländliche Räume werden von Beschäftigten nicht überproportional häufig verlassen. Die regionale Arbeitsmarktlage einer Region erzeugt allerdings altersselektive Abwanderungsmuster: Jüngere Beschäftigte verlassen mit höherer Wahrscheinlichkeit strukturschwache Regionen. Dieser Befund legt nahe, dass die Entscheidung zu überregionaler Betriebsmobilität stark von der Position im Lebensverlauf sowie von Entscheidungen im Haushaltskontext und von ortsgebundenen sozialen Netzwerken im Freundes-, Bekannten- und Verwandtenkreis abhängt.

Wenig bedeutsam sind betriebliche Charakteristika des Ausgangsbetriebs. Allerdings sind branchenspezifische Mobilitätsmuster in ähnlicher Weise, wie sie bereits für zwischenbetriebliche Mobilität gezeigt wurden (Struck 2006, S. 153), erkennbar: Für Beschäftigte in den Bereichen „Handel“ und ,unternehmensnahe Dienstleistungen“ lässt sich im Vergleich zu Personen im verarbeitenden Gewerbe eine signifikant höhere räumliche Mobilitätsneigung nachweisen. Dies könnte auf das geringere betriebsspezifische Humankapital der Beschäftigten in diesen Sektoren zurückzuführen sein (vgl. Struck et al. 2007). In Übereinstimmung mit Haas (2000) zeigen die von mir durchgeführten Analysen ein prozyklisches räumliches Mobilitätsverhalten. Im Zeitraum wirtschaftlicher Abschwünge zu Anfang und am Ende des Beobachtungszeitraumes besteht eine signifikant geringere überregionale Mobilitätswahrscheinlichkeit.

Die multinomialen Modelle zu den Einflüssen von substanziellen Einkommensveränderungen im Zuge von Betriebsmobilität zwischen Raumordnungsregionen stützen im Grundsatz ökonomischrational argumentierende, nutzentheoretische Erklärungen auf Basis des place-utility-Ansatzes (Wolpert 1965) sowie suchtheoretische Überlegungen (Kalter 1994; McKenna 1985). Häufig sind jene Gruppen tatsächlich erfolgreich, die auch eine höhere Mobilitätsneigung aufweisen. Dies sind insbesondere jüngere und qualifizierte Beschäftigte. Daneben begünstigen Groß- und Hochlohnbetriebe Aufstiegschancen und schützen vor Einkommensverlusten. 
Der Blick auf regionale Merkmale zeigt, dass überregionale Arbeitsplatzwechsel in wirtschaftlich prosperierende Zielregionen unter Berücksichtigung des Preisniveaus kein Garant für Einkommenszuwächse sind. Direkt nach dem Wechsel in vergleichsweise wirtschaftlich leistungsstarke Regionen sowie in Räume mit einer hohen Konzentration von hochqualifizierten Beschäftigten sind geringe Aufstiegschancen zu beobachten. Eine hohe regionale Humankapitalkonzentration erhöht die Wahrscheinlichkeit von Einkommensverlusten. Auch für Hochqualifizierte besteht in diesen Räumen eine höhere Wahrscheinlichkeit Abstiege in Kauf nehmen zu müssen. Dass in Regionen mit einem vergleichsweise hohen Anteil an hoch qualifizierten Beschäftigten Erwerbsrisiken bestehen, ist bereits für überbetriebliche Mobilität festgestellt worden. Offensichtlich besteht in diesen Räumen generell eine höhere Marktkonkurrenz. Hochqualifizierte können das Potential ihrer Qualifikation unter diesen Bedingungen entgegen den Überlegungen der „,skill-biased technological and organisational change“-Ansätze (Acemoglu 2002; Bresnahan et al. 2002) wohl nicht bzw. nicht unmittelbar ausschöpfen, da der Spielraum für Einkommenssteigerungen durch die höhere Konkurrenz zwischen den Hochqualifizierten begrenzt ist.

Nachdem die Einfluss- und Erfolgsfaktoren von Betriebsmobilität auf der Ebene von Raumordnungsregionen aufgezeigt wurde, stellt sich aufgrund der erheblichen Disparitäten zwischen Ost-, West- und Nord- und Süddeutschland und der Folgen der Wiedervereinigung die Frage, ob und inwieweit die Mobilitäts- und Einkommenserfolgsmuster für überbetriebliche Mobilitätsprozesse zwischen Ost-, Nord- und Süddeutschland gelten.

\subsection{Erkenntnisse aus dem Fachartikel „Regional Mobility and Spatial Inequality: Deter- minants of Spatial Labor Market Behavior Considering Firm- and Region-Specific} Factors"

So wurden in einem nächsten Schritt die Einflussfaktoren von Arbeitsortmobilität auf der Ebene von Großregionen in dem Artikel „Regional Mobility and Spatial Inequality: Determinants of Spatial Labor Market Behavior Considering Firm- and Region-Specific Factors“" (Ganesch 2018), der in der Zeitschrift Raumforschung und Raumordnung veröffentlicht wurde, analysiert.

Dabei kann analog zu den Ergebnissen von räumlichen Mobilitätsprozessen auf der Ebene von Raumordnungsregionen gezeigt werden, dass auch Arbeitsplatzwechsel zwischen den Großregionen Nord-, Ost- und Süddeutschland in erster Linie durch die Individualmerkmale Alter und beruflicher Qualifikation determiniert werden (Windzio 2004a; Hacket 2009). Von betrieblichen 


\section{Darstellung und Interpretation der eigenen Erkenntnisbeiträge}

Merkmalen gehen - anders als bei betrieblicher Mobilität insgesamt - bei Mobilität zwischen Großregionen kaum eigenständige Effekte aus. Regionale Kontextfaktoren sind insofern bedeutsam, als sie in Abhängigkeit vom Lebensalter und dem Qualifikationsniveau der Beschäftigten Relevanz besitzen. Demnach werden Regionen mit einem hohen Arbeitslosigkeitsniveau insbesondere von Jüngeren und Geringqualifizierten verlassen, hingegen weniger von älteren Beschäftigten und Arbeitskräften mit einem beruflichen oder akademischen Abschluss. Demnach legen die Ergebnisse zu Betriebsmobilität zwischen Großregionen analog zu den Befunden zu überregionaler Betriebsmobilität auf der Ebene von Raumordnungsregionen nahe, dass mit zunehmenden Alter die familiale und soziale Bindung an einen Ort höher ist und einen höheren Stellenwert besitzt als berufliche Ambitionen außerhalb des Beschäftigungsbetriebs. Hochqualifizierte konkurrieren nicht oder deutlich seltener mit Arbeitslosen um Stellen, Einkommen oder Arbeitsbedingungen, als dies bei Geringqualifizierten der Fall ist. Gemäß dem place utility-Ansatz (Wolpert 1965) sind regionale Strukturindikatoren, wie die lokale Arbeitsmarktsituation, für Mobilitätsentscheidungen nicht objektiv betrachtet relevant, sondern deren subjektive Wahrnehmung und Bewertung vor dem Hintergrund der individuellen Ressourcen und beruflichen und privaten Ziele.

Die Ergebnisse verdeutlichen entgegen der Erwartung, dass kein übermäßiger Abstrom von hochqualifizierten Beschäftigten aus strukturschwachen Regionen erfolgt. Spezifischere Analysen deuten zudem an, dass ostdeutsche Agglomerationsräume wie Berlin, Jena, Leipzig, etc. Beschäftigte mit einem Hochschulabschluss binden können. Gleichwohl besteht ein Problem für diese Regionen darin, dass altersselektive Abwanderungsmuster strukturarme Gebiete unmittelbar und nachhaltig schwächen. Strukturschwache Regionen befinden sich insbesondere in Ostdeutschland. Durch die Abwanderung junger Arbeitskräfte ist deren Anteil an der lokalen Bevölkerung überproportional gesunken. Dies ist insofern von Bedeutung, als die regionale Altersverteilung Auskunft darüber gibt, inwieweit den Unternehmen der Zugang zu jungen qualifizierten Arbeitskräften und innovativem Wissen möglich ist (Fink und Tiemann 2016). Sind junge Menschen anteilig unterrepräsentiert, hat dies unmittelbar eine negative Signalwirkung. Hinzu kommt, dass in regionale Arbeitsmärkte, die infolge altersselektiver Wanderungsverluste deformierte Altersstrukturen aufweisen, auch künftig deutlich weniger junge Menschen eintreten werden. Dies gilt auch dann, wenn die Abwanderungsneigung abnimmt (Windzio 2013, S. 670). Folgt man der These, dass die Anteile junger Erwerbspersonen als Indikator für das zukünftige wirtschaftliche Leistungs-, Innovations- und Wachstumspotential gelten (Fink und Thiemann 2016; Niedomys1 
und Hansen 2010; Ryder 1965), mindern derartige Alterskonstellationen die Standortattraktivität langfristig. Aufgrund der altersselektiven Wanderbewegungen aus strukturarmen Räumen sprechen unsere Befunde nicht für eine Angleichung regionaler Disparitäten, sondern deuten eine Reproduktion, wenn nicht sogar eine Verstärkung bestehender raumstruktureller Ungleichheiten an. Dies gilt insbesondere für die Großregionen Nord-, Ost- und Süddeutschland. Es gilt aber auch innerhalb dieser Regionen für Raumordnungsregionen.

Selbst wenn die vorgestellten Untersuchungsbefunde lediglich regionale und überregionale Betriebswechsel von (Vollzeit-)Beschäftigten in den Blick nehmen, die Wohnortmobilität von derzeit nicht-erwerbstätigen Gruppen (Arbeitsmarkteinstiege von Absolvent(inn)en, die eine wesentliche Zielgruppe für raumstrukturelle Veränderungen sind,) ausklammern und somit nur eingeschränkt raumstrukturelle Prognosen zulassen, stimmen die skizzierten, raumbezogenen Schlussfolgerungen und Entwicklungstendenzen unserer Analyse mit den zeitlich auf unsere Ergebnisse folgenden, tatsächlichen Raumbeobachtungen (INKAR-Strukturindikatoren BBSR) überein. Die regionale demografische Situation bzw. die Entwicklung der Altersstruktur variiert deutlich zwischen den Landesteilen (siehe Anhang Abbildung A1 und A2 ${ }^{15}$ ). So sank der Anteil der Einwohner im Alter von 18 bis unter 25 Jahren von 2000 bis 2014 in Ostdeutschland von 9\% auf ca. 5\%, während der Anteil dieser Gruppe der jungen Erwachsenen in Süd- und Norddeutschland im Betrachtungszeitraum sogar leicht ansteigt und auf einem Niveau von ungefähr $8 \%$ verbleibt. Eine ähnliche Entwicklung lässt sich auch für junge sozialversicherungspflichtig Beschäftigte (im Alter von 15 bis unter 30 Jahren) beobachten.

Inwiefern Betriebsmobilität zwischen Ost-, Nord- und Süddeutschland die Wahrscheinlichkeit von Einkommenszuwächsen beeinflusst, ist Gegenstand des nachfolgenden Beitrages.

\subsection{Erkenntnisse aus dem Fachartikel „Arbeitsplatzmobilität zwischen Ost-, Nord- und Süddeutschland: Erfolgsfaktoren von Einkommenszuwächsen“}

Es ist davon auszugehen, dass Betriebsmobilität zwischen Landesteilen mit erheblichen ökonomischen und sozialen Kosten sowie kulturellen Veränderungen einhergeht. Diesen stehen wiederum Erwartungen von Einkommenszuwächsen entgegen. Vor diesem Hintergrund wurden in ei-

\footnotetext{
${ }^{15}$ Eigene Berechnungen auf Basis der INKAR-Strukturindikatoren des BBSR.
} 


\section{Darstellung und Interpretation der eigenen Erkenntnisbeiträge}

nem weiteren Beitrag „Arbeitsplatzmobilität zwischen Ost-, Nord- und Süddeutschland. Erfolgsfaktoren von Einkommenszuwächsen“, der im Frühjahr 2020 in der Zeitschrift Sozialer Fortschritt erscheinen wird, die Bedingungen für Einkommenserfolg aller Betriebsmobilen in den Blick genommen. In multinomialen Schätzmodellen werden Individualmerkmale, regionale Strukturindikatoren auf der Ebene der Zielraumordnungsregion, das betrieblichen Lohnniveau sowie der Wirtschaftszweig des Zielbetriebs berücksichtigt.

Unter Kontrolle der Ausgangsregion, der Entfernung zu einer Großregion und individueller Merkmale sowie der betrieblichen und regionalen Charakteristika des jeweiligen Zielbetriebskontextes ist festzustellen, dass Arbeitsortmobilität zwischen Großregionen die Wahrscheinlichkeit substanzielle Einkommenserfolge zu erzielen, im Vergleich zu lokalen Betriebswechseln erwartungsgemäß erhöht. Werden die Wanderungsrichtungen zwischen den Großregionen Nord-, Süd- und Ostdeutschland berücksichtigt, so zeigen sich für Wechsel aus Ostdeutschland in Richtung Nordund in Richtung Süddeutschland preisbereinigt höhere Einkommenserfolgschancen als für Betriebsmobile innerhalb Ostdeutschlands. Die für die Folgejahre der deutschen Wiedervereinigung festgestellten Einkommenschancen bei Ost-West-Mobilität (Hacket 2009) bestehen bis 20 Jahre nach der Wende und auch bei Berücksichtigung regionaler Preisunterschiede fort. Eine Ursache können nach wie vor bestehende problematischere Wiederbeschäftigungsmöglichkeiten aufgrund der wirtschaftlichen Situation und der meist größeren Konkurrenz am Arbeitsmarkt in Ostdeutschland sein, die dann besonders bei unfreiwilligen Betriebswechseln negative Folgen haben. Arbeitsplatzwechsel in Regionen Nord- oder Süddeutschlands erfolgen häufiger aufstiegsorientiert. Zudem sind Einkommenserfolge für in Süd- und Norddeutschland Beschäftigte, die innerhalb der Großregion betriebsmobil sind und diejenigen, die die Großregion - unabhängig von der Zielgroßregion - verlassen, wahrscheinlicher als für Erwerbstätige, die ihren Arbeitgeber innerhalb Ostdeutschlands wechseln. Arbeitsortmobilität von Nord- oder Süddeutschland in Richtung Ostdeutschland zieht jedoch ambivalente Einkommenseffekte nach sich, da sie ebenfalls mit einer vergleichsweise höheren Wahrscheinlichkeit von Einkommensverlusten einhergehen kann.

Individualmerkmale stimmen weitgehend mit bestehenden empirischen Befunden (Lehmer und Ludsteck 2011, S. 558, 565; Reichelt und Abraham 2015) und den eigenen Ergebnissen, die für die Gruppe der Regionalmobilen auf der Ebene von Raumordnungsregionen gezeigt werden konnten (Ganesch et al. 2019), überein. Abstiegsrisiken lassen sich für geringer Qualifizierte und 


\section{Darstellung und Interpretation der eigenen Erkenntnisbeiträge}

für Ausländer nachweisen. Die Ergebnisse sprechen nicht dafür, dass die qualifikationsspezifischen Einkommenserfolgsaussichten bei Betriebsmobilität durch das Verlassen einer Großregion verstärkt werden.

Entgegen unserer Erwartung aber im Einklang mit dem Befund für betriebsmobile Beschäftigte auf der Ebene von Raumordnungsregionen (Ganesch et al. 2019) mindern Wechsel in wirtschaftlich leistungsstarke Zielräume, gemessen am Bruttoinlandsprodukt der jeweiligen Raumordnungsregion und bei Kontrolle auch betrieblicher Faktoren die Wahrscheinlichkeit von Einkommenserfolge. Der individuell (verhandelte bzw. akzeptierte) Lohn sowie das Lohnniveau in der Zielbetriebsstätte sind bedeutsamer für Einkommenszuwächse nach betrieblicher Mobilität, als das wirtschaftsstrukturelle Umfeld der gewählten Zielregion. Allerdings ist hierbei wiederum zu berücksichtigen, dass Einkommenseffekte unmittelbar nach dem Betriebswechsel betrachtet wurden. Möglicherweise treten positive Effekte, die ggf. beim Zuzug in urbane Räume mit hoher wirtschaftlicher Leistungsfähigkeit erwartet wurden, später ein. ${ }^{16}$ In Anlehnung an den Befund von Kelle (2016) kann die Relevanz betrieblicher Kontexte im Hinblick auf die Betrachtung individueller Einkommenschancen herausgestellt werden. Mit Blick auf Wirtschaftszweige ist festzustellen, dass Wechsel in den Wirtschaftssektor unternehmensnahe Dienstleistungen mit erhöhter Wahrscheinlichkeit von Einkommensverlusten einhergehen.

Insgesamt können ökonomisch-rational argumentierende Ansätze, nach denen zu erwarten ist, dass Vollzeiterwerbstätige in ihrer Mehrzahl durch direkte Betriebswechsel in entfernte Großregionen keine Einkommenseinbußen hinnehmen wollen, bestätigt werden. Es zeigt sich aber auch, dass betriebliche Mobilität zwischen den Landesteilen auch erfolgt, wenn unmittelbar keine Einkommenszuwächse realisiert werden können, was einem eng verstandenen ökonomischen Kalkül entgegensteht. So verlassen einzelne Beschäftigtengruppen Betriebe unfreiwillig und/oder haben kaum Wahlmöglichkeiten beim Einstieg in ein neues Beschäftigungsverhältnis. Dies deutet sich bei geringer qualifizierten Beschäftigten, bei Ausländern und im Falle von Betriebswechseln innerhalb Ostdeutschlands an, da diese mit einer etwas höheren Wahrscheinlichkeit Einkommenseinbußen sowie mit einer vergleichsweise geringeren Wahrscheinlichkeit Einkommenszuwächse

\footnotetext{
${ }^{16}$ Über die Beiträge hinausgehende Analysen (Anhang Tabelle A1; Tabelle A2) zeigen jedoch, dass wirtschaftsstarke Raumordnungsregionen zwei Jahre nach dem Betriebswechsel die Chance, Einkommenszuwächse zu realisieren, zumindest nicht signifikant verringern. Bezeichnend ist, dass von Räumen mit einer vergleichsweise hohen Wirtschaftskraft auch nach zwei Jahren kein Einkommenszuwachs fördernder Effekt ausgeht unter der Kontrolle von erwerbsrelevanten Individualmerkmalen sowie betrieblicher und regionaler Kontextvariablen.
} 


\section{Darstellung und Interpretation der eigenen Erkenntnisbeiträge}

haben. Eine Aufklärung hierüber wäre allerdings auf eine umfänglichere Berücksichtigung von Rahmenbedingungen angewiesen, die über erwerbsbezogenen Merkmale hinausgeht und etwa familiäre und soziale Kontexte sowie ortsgebundene Ressourcen miteinbezieht. Private Gründe, etwa familiären Erwägungen, unter Umständen auch Rückmobilität an einen Ort oder in eine Region, in der auf familiale und soziale Ressourcen zurückgegriffen werden kann, können berufliche Aufstiegs- und Weiterentwicklungsmotive überlagern und dazu führen, dass betriebliche Mobilität nicht primär beruflich- bzw. aufstiegsmotiviert erfolgt (Glorius 2010; Hacket 2009, S. 119). Derartige Aspekte und Konstellationen können auf Basis der der Analyse zugrundeliegenden, verknüpften Erwerbs-, Betriebs- und Regionaldaten, die ihre Stärke in der präzisen Erfassung von Beschäftigungszeiten und Erwerbseinkommen im betrieblichen und regionalwirtschaftlichen Kontext hat, nicht kontrolliert werden.

Im folgenden Abschnitt werden die wesentlichen inhaltlichen Erkenntnisbeiträge resümierend festgehalten und aus einem theoretischen Blickwinkel sowie hinsichtlich ihrer raumstrukturellen Implikationen beleuchtet. 


\section{Zusammenfassung und Fazit}

Im Zuge wirtschaftlicher und sozialer Anpassungsprozesse und ausgehend von räumlichen Disparitäten am Arbeitsmarkt betrachtet die Synopsis zwischenbetriebliche und überregionale Mobilitätsprozesse und ihre Konsequenzen in Bezug auf die individuelle Einkommensentwicklung, d.h. mit dem Betriebswechsel einhergehende substanzielle Einkommensgewinne oder-verluste. Bestehende Analysen berücksichtigen insbesondere die individuellen Determinanten (Bender et al. 2000; Boockmann und Steffes 2010; Fitzenberger und Garloff 2007; Grotheer et al. 2004; Struck et al. 2007). Betriebliche Beschäftigungsstabilität wie auch die Entscheidung, den Arbeitgeber zu wechseln, sind jedoch in betriebliche und regionale Kontexte eingebunden. Betriebsmerkmale und regionale Charakteristika werden aber bislang selten systematisch in empirische Untersuchungen einbezogen. Hinsichtlich überregionaler Betriebsmobilität liegen den meisten bisherigen Untersuchungen aggregatsstatistische oder auf bestimmte Regionen und Beschäftigtengruppen bezogene Betrachtungen zugrunde.

Die vorliegende Synopsis setzte an diesem Punkt an und führt dazu die Erkenntnisbeiträge zu folgenden Fragen, die in den vier Beiträgen untersucht wurden, zusammen:

- Welche Bedeutung haben regionale und betriebliche Kontextmerkmale in Bezug auf die betriebliche Beschäftigungsstabilität? Welche Einkommens- und Erwerbschancen gehen mit der zwischenbetrieblichen Mobilität der Beschäftigtengruppen einher?

- Was sind die individuellen, betrieblichen und regionalen Determinanten und Erfolgsfaktoren von überregionalen Betriebswechseln auf der Ebene von Raumordnungsregionen?

- Von welchen individuellen, betrieblichen und regionenspezifischen Merkmalen werden Betriebswechsel zwischen Ost-, Nord- und Süddeutschland beeinflusst?

- Welchen Einfluss haben überregionale Betriebswechsel zwischen Ost-, Süd- und Norddeutschland auf die individuelle Einkommensentwicklung unter der Kontrolle von individuellen, betrieblichen und regionalen Einflüssen?

Die Synthese der Befunde leistet neben dem inhaltlichen Erkenntnisbeitrag einen Beitrag zum theoretischen Erklärungsmodell betrieblicher Mobilitätsprozesse im regionalen Kontext, gibt Auskunft über die räumliche Dimension erwerbsbezogener sozialer Ungleichheit und lässt vorsichtig zu interpretierende Aussagen in räumlicher Hinsicht zu. 
Inhaltlich wurde in dieser Synopsis verdeutlicht, unter welchen betrieblichen und regionalen Gegebenheiten unterschiedliche Beschäftigtengruppen versuchen, ihre eigenen Beschäftigungsbedingungen durch regionale und überregionale betriebliche Mobilität zu verändern und unter welchen Bedingungen ein unmittelbarer Einkommenszuwachs wahrscheinlich ist. Über den bestehenden Forschungsstand hinausgehend konnte ich zeigen, dass die individuellen Erwerbschancen und Einkommenschancen neben den individuellen arbeitsmarktbezogenen Merkmalen der Beschäftigten von betrieblichen und regionalen Kontextmerkmalen beeinflusst werden. Bedeutsam sind betriebsstrukturelle Merkmale im Sinne segmentationstheoretischer Überlegungen. Betriebliche Prosperitätsfaktoren (Lohnniveau, erwartetes Geschäftsvolumen, Personalentwicklung, etc.), branchenspezifische Muster, etc. und Konjunktureffekte haben Einfluss auf die betriebliche Beschäftigungsstabilität und die Positionierungschancen nach einem Betriebsaustritt.

Mit Blick auf regionale Kontexte zeigt sich, dass Agglomerationsräume von einer geringeren betrieblichen Beschäftigungsstabilität gekennzeichnet sind. Gleichzeitig lassen sich hier aber verglichen mit ländlichen Gebieten mit einer höheren Wahrscheinlichkeit direkte Anschlussbeschäftigungen in Vollzeit sowie Einkommenserfolge realisieren. Aussichtsreiche Erwerbschancen haben in agglomerierten Räumen vor allem berufliche qualifizierte Beschäftigte. Mit zunehmender regionaler Humankapitalakkumulation erhöhen sich meinen Ergebnissen zufolge die Erwerbsrisiken und verringern sich die Einkommenschancen. Dies gilt sowohl für Betriebswechsel innerhalb von Regionen mit einer hohen Qualifikationsausstattung als auch für überregionaler Betriebsmobilität in Richtung dieser Zielräume (insbesondere für Hochqualifizierte). Vermutlich mindert die höhere Marktkonkurrenz zunächst die Einkommenschancen. Die regionale Arbeitslosigkeit, die wirtschaftliche Leistungsfähigkeit und das regionale Lohnniveau sind per se für überregionale Arbeitgeberwechsel und damit einhergehende substanzielle individuelle Einkommensveränderungen überraschend wenig bedeutsam. Lediglich differenziert nach Alters- und Qualifikationsgruppen zeigen sich für das regionale Arbeitslosigkeitsniveau signifikante Effekte. Hochschulabsolventen in Regionen mit einer vergleichsweise hohen Arbeitslosigkeit weisen kein signifikant abweichendes Mobilitätsmuster auf. Sie treten einerseits in der Regel nicht in Konkurrenz zu zumeist geringer qualifizierten Arbeitslosen. Andererseits können sie offenbar auf Basis ihrer Qualifikationen auch in weniger prosperierenden Regionen ein hinreichendes Auskommen finden. Für jüngere und mittlere Altersgruppen ist im Vergleich zu über 50-jährigen Beschäftigten ein Verlassen strukturschwacher Regionen wahrscheinlicher. 
Auf Basis der zugrunde gelegten Datenlage und deren Einschränkungen ${ }^{17}$ sind rational-ökonomisch argumentierende Ansätze weitgehend zu bestätigen. Es finden sich aber auch Beschäftigtengruppen, wie Geringqualifizierte und Beschäftigte mit Arbeitslosigkeitserfahrungen, mit erhöhten Einkommensverlustrisiken. Mögliche Erklärungen hierfür könnten die Unfreiwilligkeit des Betriebsaustritts und/oder die geringeren Wahlmöglichkeiten beim Einstieg in ein neues Beschäftigungsverhältnis sein. Hinzukommt, dass Einkommenseffekte unmittelbar nach dem Betriebswechsel betrachtet werden. Teilweise sehen wir, dass sich Wechsel in Agglomerationsräume und wirtschaftsstarke Räume finanziell nicht direkt auszahlen. Möglicherweise ergibt sich ein anders Bild bei späteren Erträgen ${ }^{18}$

Hinsichtlich der Struktur des Arbeitsmarktes legt die vorliegende Untersuchung betrieblicher und überregionaler Mobilitätsprozesse analog zu bestehenden empirischen Befunden (Blien et al. 2006; Farhauer und Granato 2006; Fassmann und Meusburger 1997) nahe, dass regionale Teilarbeitsmärkte existieren. In Übereinstimmung mit Kelle (2016) beeinflussen jedoch auch betriebsspezifische Merkmale die Einkommens- und Beschäftigungschancen. Ebenfalls segmentationstheoretischen Überlegungen entsprechend sind qualifikationsspezifische Erwerbsmuster festzustellen.

Ausgehend vom Kernbefund der Untersuchung, dass neben den bedeutsamen individuellen und betriebsstrukturellen Determinanten auch regionale Kontextfaktoren die Chancen und Risiken im Erwerbsverlauf beeinflussen, kann in theoretischer Hinsicht festgehalten werden, dass durch die Einbindung struktureller Einflussfaktoren in die bestehenden und zumeist individualistisch ausgerichteten und ökonomisch-rational argumentierenden Arbeitsmarkttheorien deren Erklärungskraft in Bezug auf betriebliche Mobilitätsprozesse gesteigert werden kann. Mit den in dieser Arbeit vorgestellten konzeptionellen Erweiterungen, die die theoretischen Überlegungen von Coleman (1990) und Esser (1996) aufgreifen, indem sie die Rahmenbedingungen sozialer Prozesse umfassend im Analysemodell abbilden, wurde auf bereits bestehende Ausführungen in der regionalökonomischen Forschung zurückgegriffen (Fujita et al. 2001; Krugman 1991). Damit sollten

\footnotetext{
${ }^{17}$ Betrachtet wurden lediglich Vollzeitbeschäftigte mit einem Einkommen, das unterhalb der Beitragsbemessungsgrenze der gesetzlichen Rentenversicherung liegt. Beschäftigte mit einem Einkommen, das jenseits der Beitragsbemessungsgrenze der gesetzlichen Rentenversicherung liegt, sowie der Personen, die sich nicht in einer sozialversicherungspflichtigen Vollzeitbeschäftigung befinden, waren demnach nicht Gegenstand der Analyse.

${ }^{18}$ Die über die Artikel hinausgehende Untersuchung der Einkommensentwicklungen im weiteren Erwerbsverlauf zeigt, dass Agglomerationsräume und Regionen mit Verstädterungstendenzen zwei Jahre nach dem Betriebswechsel Einkommenszuwächse begünstigen (siehe Anhang Tabelle A2). Dieser Effekt findet sich jedoch nicht für regionalmobile Beschäftigte (siehe Anhang Tabelle A1).
} 


\section{Zusammenfassung und Fazit}

unter Einbeziehung der regionalen Arbeitsmarkt- und Wirtschaftsstrukturen expliziter die auf regionaler Ebene bestehenden Angebots- und Nachfragerelationen sowie die regionalen Gelegenheitsstrukturen berücksichtigt werden, die eine unmittelbare Auswirkung auf die Erwerbsverläufe der Beschäftigten haben (Dütsch und Struck 2014, S. 40f.). Insgesamt erwies sich der Versuch, regionalökonomische Ansätze in das handlungstheoretische Entscheidungsmodell zu integrieren, als begrenzt fruchtbar. Denn die Art und Weise wie regionale Rahmenbedingungen auf individuelle Erwerbsverläufe wirken, wird von Individualmerkmalen moderiert, die anhand von Alterund Qualifikationsgruppen nur näherungsweise abgebildet werden können. Individuelle Raumbewertungen werden gemäß dem place utility-Ansatz (Wolpert 1965) vor dem Hintergrund der Lebenssituation und der Lebensziele, der Partnerschafts- und Haushaltskonstellation sowie der ortsgebundenen Ressourcen getroffen.

In der empirischen Analyse noch nicht ausreichend aufgegriffen wurde die lebensverlaufstheoretische Perspektive (Blossfeld und Huinink 2001; Kohli 1985; Mayer 1990), und damit verbunden nicht-berufsbedingte Einflüsse von Betriebsmobilität. So bestehen Wechselwirkungen zwischen den Lebensbereich Arbeit und dem privaten Lebensbereich. Biografische Ereignisse, die individuelle Lebenssituation und die Lebensziele, partnerschaftliche und familiäre Bindungen und ortsgebundene Ressourcen beeinflussen in Verbindung mit den regionalen Kontextfaktoren Mobilitätsentscheidungen im Lebens- und Erwerbsverlauf maßgeblich (Huinink und Kley 2008). Diverse Forschungsarbeiten legen dar, dass Familien- und Haushaltsarrangements für die Erwerbsstrategie und Karriereorientierung (Buchholz und Grunow 2006; Engelhardt 2009; Jacob und Kleinert 2014; Leitner et al. 2004) und für das räumliche Arbeitsmarktverhalten (Schneider et al. 2002a) von zentraler Bedeutung sind. Betriebswechsel müssen nicht zwingend aus beruflichen Gründen erfolgen, sondern können aus privaten Gründen, etwa familiären Erwägungen oder aufgrund eines ortbezogenen sozialen Umfeldes vorgenommen werden. Im Kontext von Partnerschaften und Familie bzw. Familienplanung finden Aushandlungsprozesse zu Art und Umfang der Erwerbsbeteiligung und Karriereorientierung statt (Auspurg und Abraham 2007; Nisic 2010). Dieser Aspekt kann mit den in diesem Projekt verwendeten verknüpften Erwerbs-, Betriebs- und Regionaldaten, die ihren Schwerpunkt und ihre Stärke in der genauen Erfassung von Erwerbsverläufen und Einkommen im betrieblichen und regionalen Kontext haben, nicht kontrolliert werden. Mobilitätserfordernisse im Lebensbereich Arbeit haben Konsequenzen für andere Lebensbereiche, wie die Familie und das soziale Umfeld von Freunden, Freizeit etc. Aus diesem Grund sind 
räumliche Mobilitätsentscheidungen am Arbeitsmarkt stets parallel zum privaten Lebensbereich, zu biografischen Schlüsselereignissen, individuellen Zielen, Präferenzen und Wünschen, die Personen dahingehend haben, zu sehen. Die berufliche und private Situation, familiäre Bindungen, berufliche und private Ziele und deren Realisierbarkeitserwartung im bestehenden oder alternativen betrieblichen und regionalen Kontext sowie ortsabhängige Ressourcen beeinflussen die Wahrnehmung regionaler Gegebenheiten und damit die Entscheidung zu regionaler und überregionaler Betriebsmobilität. Es ist davon auszugehen, dass Mobilitätsentscheidungen, die nicht rational-ökonomisch - gemessen am Einkommenserfolg - getroffen werden, durch lebensphasenspezifische Situationen und Parameter sowie Lebensziele strukturiert werden. Diese Kontexte gilt es in zukünftige Untersuchungen miteinzubeziehen.

Festzuhalten ist, wirtschaftliche und soziale Ungleichheit hat neben einer zeitlichen auch eine räumliche Dimension. Der geografische Raum weist regionale Disparitäten hinsichtlich der wirtschaftlichen Leistungsfähigkeit sowie der Arbeitsmarktlage auf. Der physische Raum spiegelt soziale Handlungen und Prozesse, durch die er geformt wird, wider und erfährt gesellschaftliche Bewertungen (Dorn 2018, S. 40). Erwerbspersonen und Beschäftigte können versuchen ihre Erwerbschancen durch räumliche Mobilität zu beeinflussen. Räumliche Mobilität verstanden als soziale Handlung ruft Veränderungen für die mobilen Personen und deren soziale Umwelt hervor und ist demnach als sozialer Prozess zu verstehen. Nach den raumtheoretischen Überlegungen von Bourdieu ist der physische Raum als angeeigneter physischer Raum immer schon ein sozial konstruierter Raum (Schroer 2006, S. 110 nach Bourdieu 1991, S. 26).

Selektive Abwanderungsprozesse, wie das Verlassen strukturschwacher Regionen von jungen Altersgruppen, ohne kompensierende Zuwanderung (in die entgegengesetzte Richtung) von jungen Menschen, führen neben einer generellen Schwächung des regionalen Arbeitskräftepotentials dazu, dass diese Altersgruppe anteilig unterrepräsentiert ist. Der eingeschränkte Zugang zu jungen, sowie besonders auch zu qualifizierten Arbeitskräften sowie zu innovativem Wissen wirkt sich dann wiederum unmittelbar negativ auf die Investitions- und Standortwahl von Betrieben in diesen Regionen aus (Fink und Tiemann 2016). Dadurch werden strukturarme Räume, wie bespielweise in Ostdeutschland nicht nur direkt, sondern auch nachhaltig geschwächt und regionalwirtschaftliche Ungleichgewichte reproduziert. Aufgrund der deformierten regionalen Altersstruktur werden auch zukünftig weniger junge Menschen in diesen regionalen Arbeitsmarkt ein- 


\section{Zusammenfassung und Fazit}

treten, auch wenn sich die Abwanderungsneigung verringert (Windzio 2013, S. 670). Da der Anteil junger Menschen in einer Region das zukünftige wirtschaftliche Leistungs-, Innovations-, und Wachstumspotential widerspiegelt (Fink und Tiemann 2016; Niedomysl und Hansen 2010; Ryder 1965), führt dies auch mittel- bis langfristig zu einer Schwächung der Standortattraktivität für Betriebe. Es ist demnach davon auszugehen, dass regionale Disparitäten durch altersselektive Abwanderungsmuster tendenziell sogar zunehmen.

Da raumstrukturelle Rahmenbedingungen Konsequenzen für individuelle berufliche Mobilitätsentscheidungen und die Standortwahl der Betriebe haben, ist regionale Strukturförderung hochrelevant. Die Untersuchungsergebnisse legen nahe, dass regionale Wirtschafts- und Strukturpolitik durchaus wirksam sein kann. Beschäftigte in ostdeutschen Zentren, etwa im Raum Berlin und Potsdam, Dresden, Leipzig und Halle oder Jena, deren bestehende lokale Wirtschaftsstruktur nach der deutschen Wiedervereinigung systematisch ausgebaut wurde und gezielt im Sinne einer Clusterpolitik (Huber 2009; Porter 2000), die soziale, qualifikatorische, technische, infrastrukturelle und wirtschaftsstrukturelle Strukturen berücksichtigt, waren deutlich weniger in Richtung Westdeutschland mobil. Zu Bedenken ist allerdings, dass sich hierbei Binnendifferenzierungen zwischen wenigen erfolgreichen Großstädten und der Peripherie innerhalb von Großregionen bzw. auch innerhalb von Bundesländern verstärken (Hinweise dazu auch in Slupina et al. 2015). Eine empirische offene Frage ist dann, ob und inwieweit die fortschreitende Digitalisierung und die damit in vielen Tätigkeitsbereichen einhergehende Möglichkeit des ortsflexiblen Arbeitens die individuelle Mobilität und die Entscheidungen zu überregionalen Betriebswechseln beeinflussen und ländliche Räume stärken können. 


\section{Anhang}

\section{Darstellung der Einzelleistungen}

Die vorliegenden Beiträge sind in Zusammenarbeit mit zwei Koautoren entstanden. Deshalb sollen im Folgenden die interne Arbeitsteilung bei der Erstellung der Beiträge sowie die jeweiligen Einzelleistungen kurz erläutert werden.

Der erste Beitrag „Employment trajectories in heterogeneous regions: Evidence from Germany“ wurde zusammen mit Matthias Dütsch und Olaf Struck (Universität Bamberg) verfasst. Dieser ist wie alle vier vorgestellten Publikationen im Rahmen des von der DFG geförderten Forschungsprojektes „Mobilität am Arbeitsmarkt. Individuelle, betriebliche und regionale Chancen- und Risikostrukturen im Erwerbsverlauf" entstanden, welches von Olaf Struck beantragt und geleitet wurde. Entsprechend ging die Ursprungsidee zu diesem Aufsatz auf Olaf Struck und Matthias Dütsch zurück. Arbeitsteilig mit Matthias Dütsch fand die Aufbereitung der LIAB-Daten statt. Die Datenauswertung und Verschriftlichung des Beitrages wurden von Matthias Dütsch und mir durchgeführt. Olaf Struck kommentierte Zwischenresultante und das Manuskript vor Einreichung. Die Datenanalyse und Verschriftlichung des zweiten Beitrages „Regionale Mobilität am Arbeitsmarkt. Individuelle, betriebliche und wirtschaftsstrukturelle Determinanten von Mobilität und Einkommen“ wurden von mir durchgeführt. Olaf Struck und Matthias Dütsch nahmen Anmerkungen zum Manuskript vor. Der dritte Beitrag „Regional Mobility and Spatial Inequality: Determinants of Spatial Labour Market Behaviour Considering Firm- and Region-Specific Factors“ wurde in Alleinautorenschaft erstellt. Die Idee zum vierten Beitrag „Arbeitsplatzmobilität zwischen Ost-, Nord-, und Süddeutschland. Erfolgsfaktoren von Einkommenszuwächsen“ wurde zusammen mit Matthias Dütsch und Olaf Struck entwickelt. Die Datenauswertung sowie die Verschriftlichung des Beitrages wurden von mir durchgeführt. Das Manuskript wurde dann wiederum in der ersten Fassung von Matthias Dütsch und Olaf Struck kommentiert. 
Abbildung A1: Anteil der sozialversicherungspflichtig Beschäftigten von 15 bis unter 30 Jahren an den sozialversicherungspflichtig Beschäftigten (in \%)

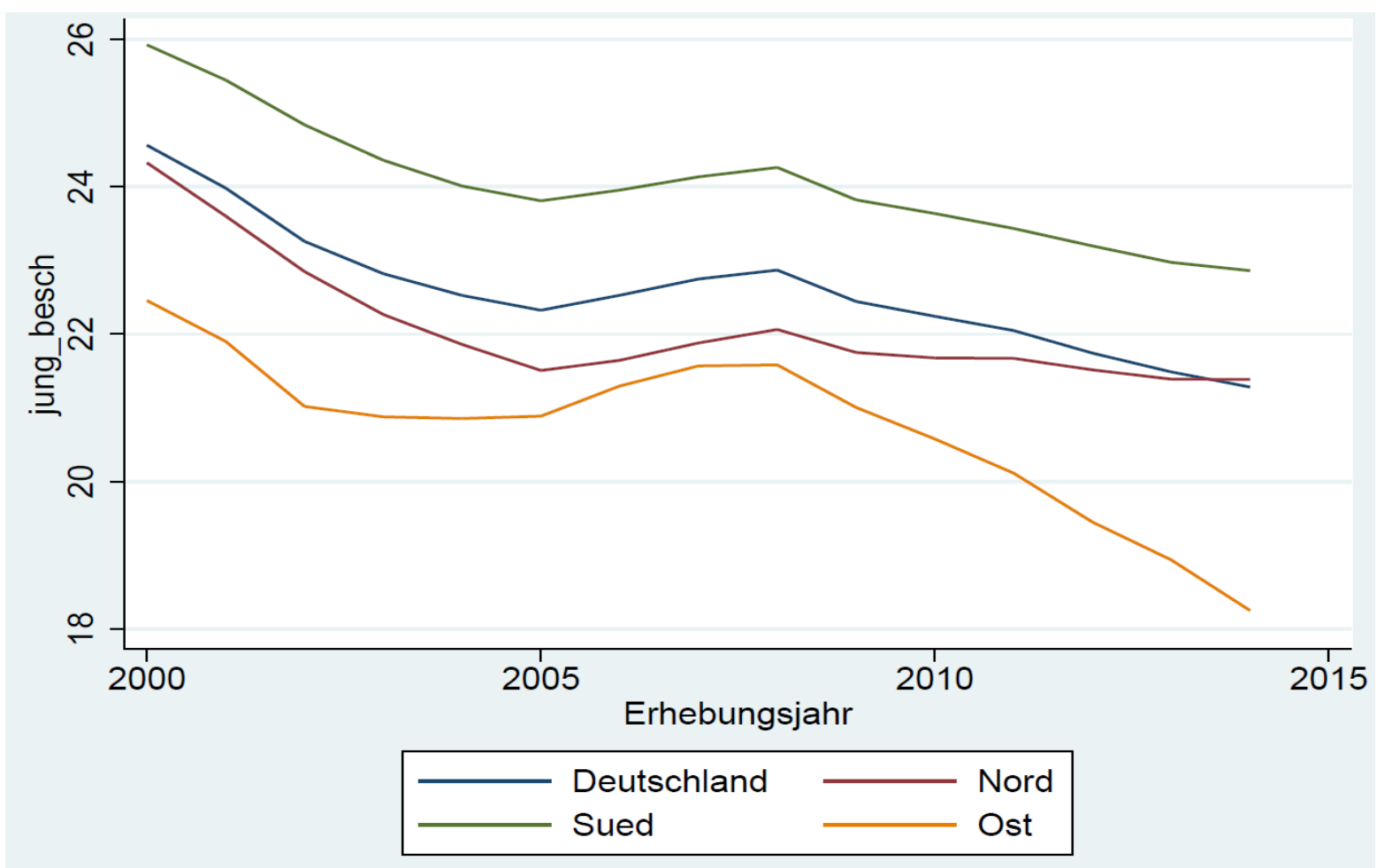

Quelle: INKAR-Strukturindikatoren (BBSR 2014): eigene Berechnung

Abbildung A2: Anteil der Einwohner von 18 bis unter 25 Jahren an den Einwohnern (in \%)

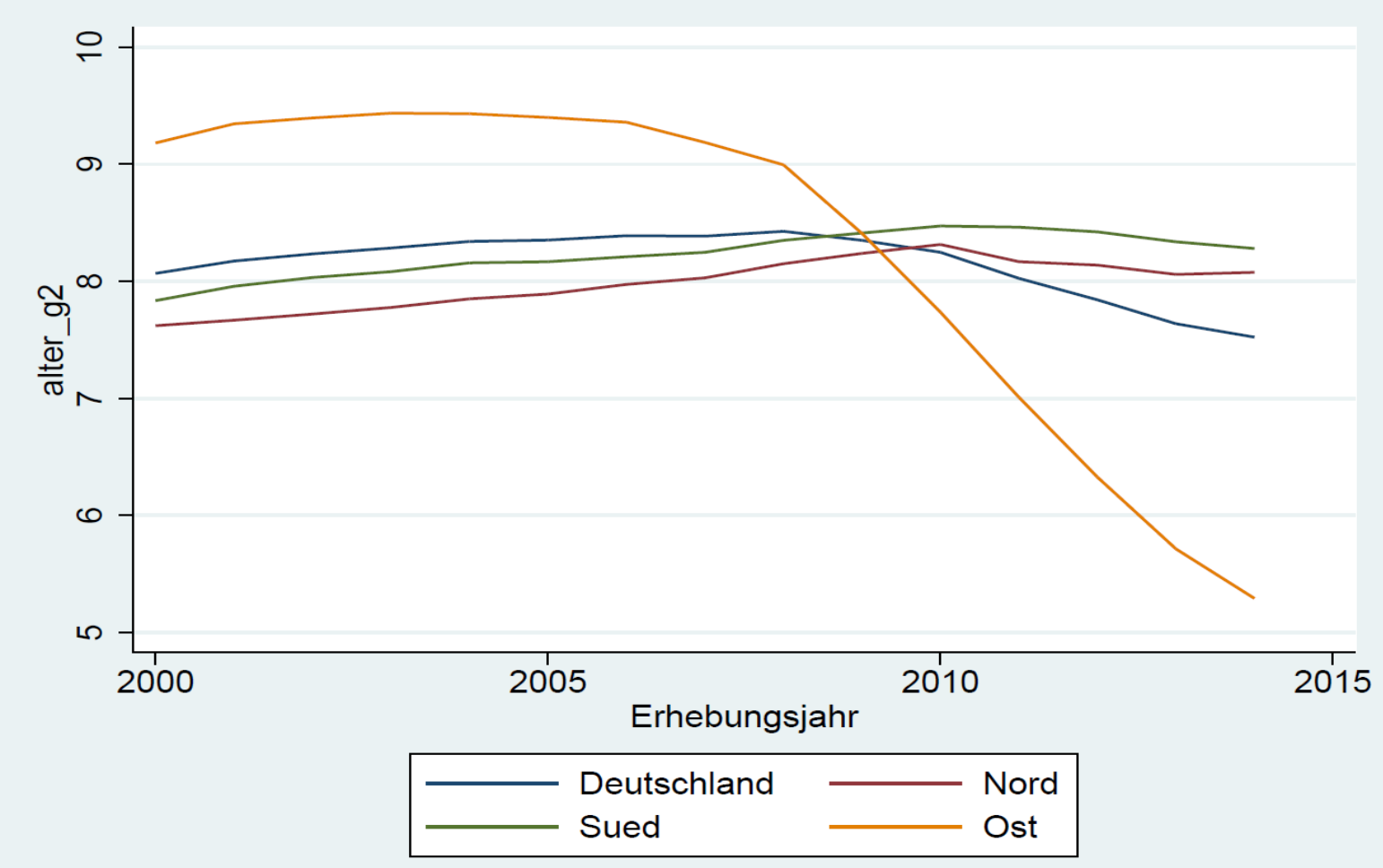

Quelle: INKAR-Strukturindikatoren (BBSR 2014): eigene Berechnung 


\section{Anhang}

Tabelle A1: Relative Risk Ratios zu den Einkommensveränderungen zwei Jahre nach dem überregionalen Betriebswechsel für Regionalmobile auf der Ebene von Raumordnungsregionen ${ }^{l}$

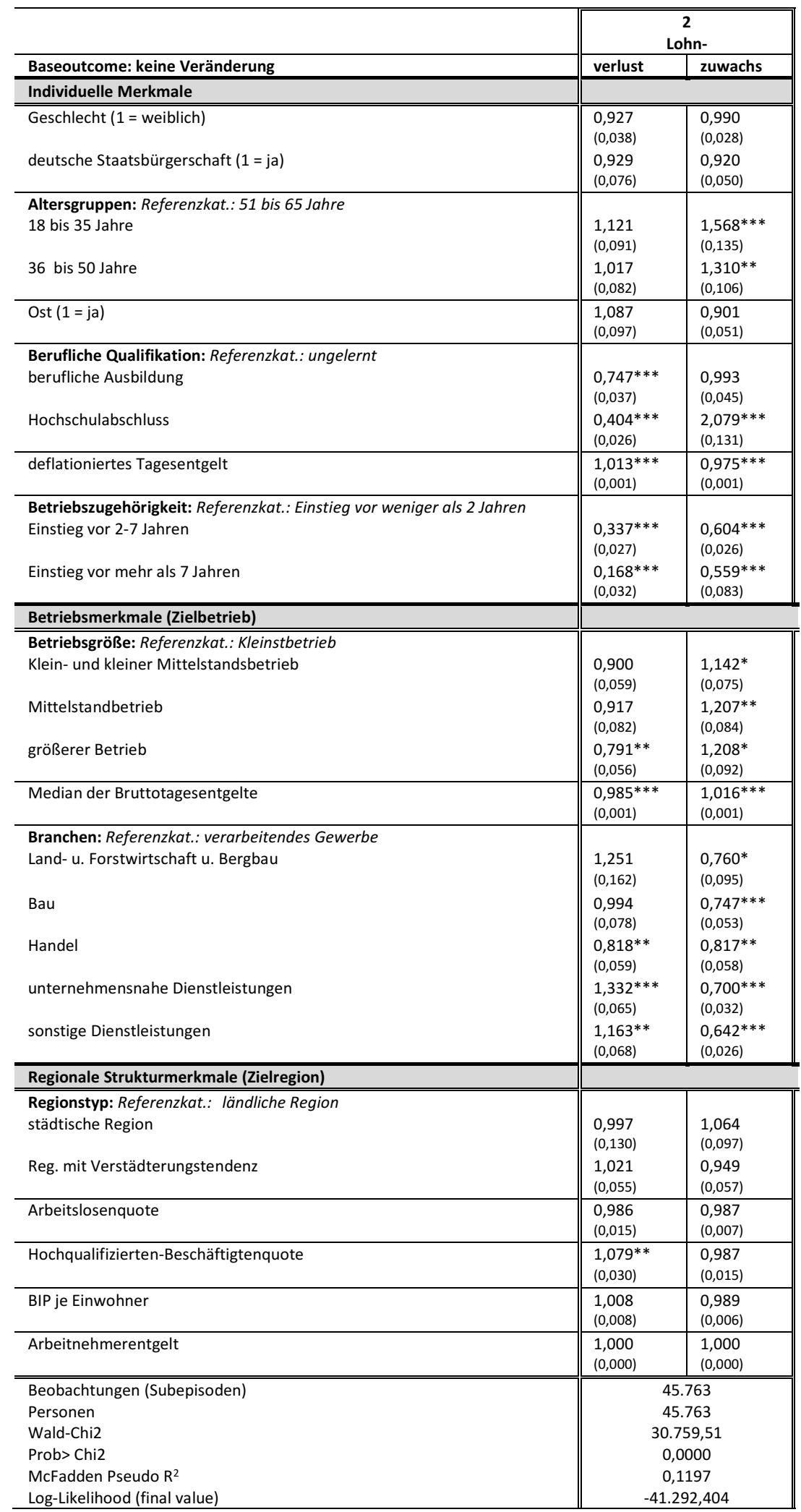

$* * * * * * *$ bezeichnet die statistische Signifikanz auf dem 0,001/0,01/0,05 Niveau, clusterrobuste Standardfehler auf der Ebene der Raumordnungsregionen Kontrolliert, aber nicht dargestellt, wurde zudem die konjunkturelle Lage mittels Jahresdummies;

Quelle: LIAB (LM 9310), eigene Berechnungen

${ }^{1}$ lokale Preisunterschiede werden berücksichtigt 


\section{Anhang}

Tabelle A2: Relative Risk Ratios zu den Einkommensveränderungen zwei Jahre nach einem Betriebswechsel (für alle Betriebsmobilen) ${ }^{2}$

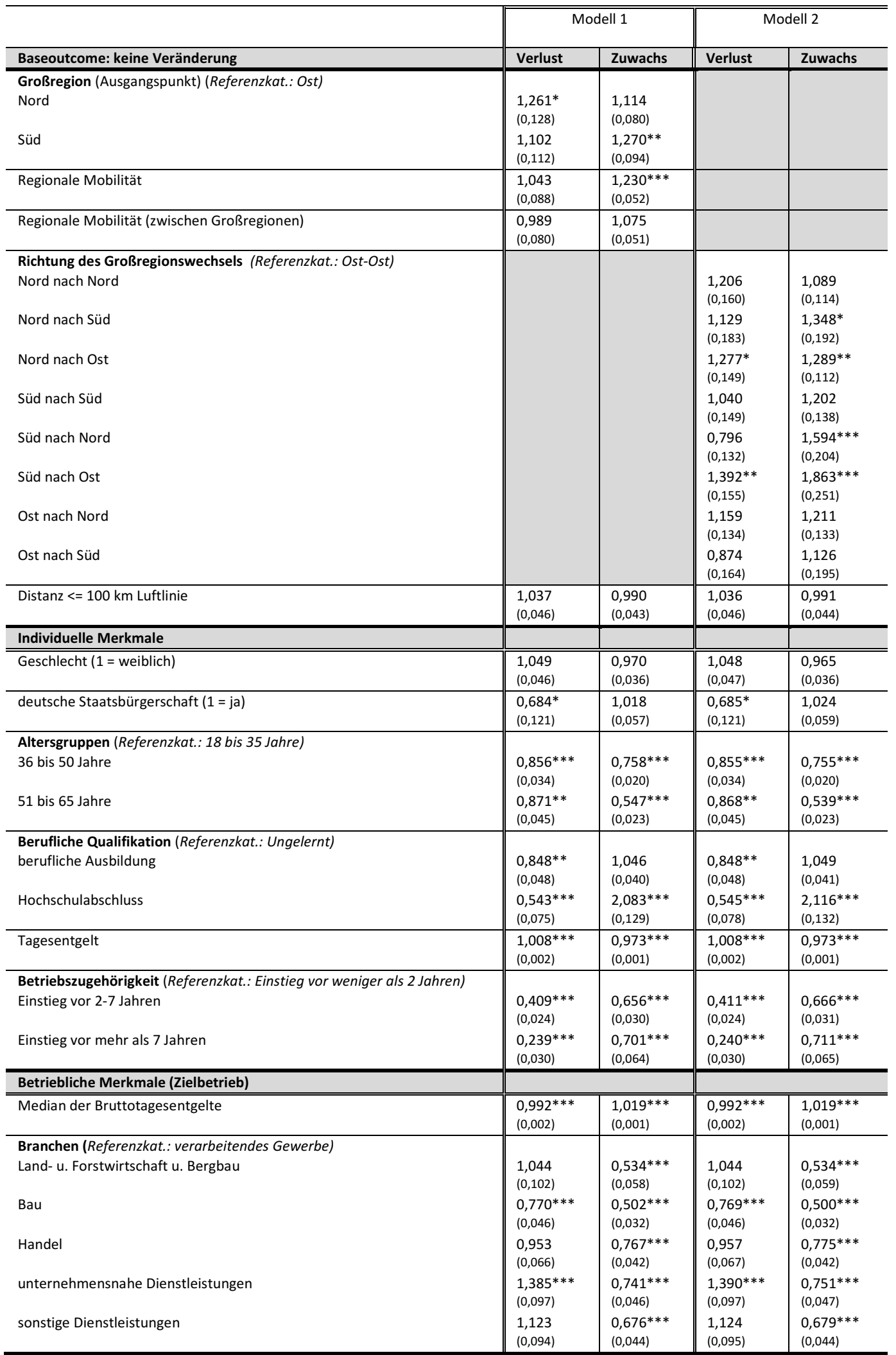

\footnotetext{
${ }^{2}$ lokale Preisunterschiede werden berücksichtigt
} 
Anhang

\begin{tabular}{|c|c|c|c|c|}
\hline \multicolumn{5}{|l|}{ Regionale Strukturmerkmale (Zielregion) } \\
\hline $\begin{array}{l}\text { Regionstyp (Referenzkat.: ländliche Region) } \\
\text { städtische Region }\end{array}$ & $\begin{array}{l}1,112 \\
(0,118\end{array}$ & $\begin{array}{l}1,286^{* *} \\
(0,123)\end{array}$ & $\begin{array}{l}1,121 \\
(0,127)\end{array}$ & $\begin{array}{l}1,281^{*} \\
(0,123)\end{array}$ \\
\hline Region mit Verstädterungstendenz & $\begin{array}{l}1,013 \\
(0,094)\end{array}$ & $\begin{array}{l}1,198^{* *} \\
(0,081)\end{array}$ & $\begin{array}{l}1,017 \\
(0,097)\end{array}$ & $\begin{array}{l}1,197^{* *} \\
(0,081)\end{array}$ \\
\hline Arbeitslosenquote & $\begin{array}{l}0,982 \\
(0,013) \\
\end{array}$ & $\begin{array}{l}1,000 \\
(0,009) \\
\end{array}$ & $\begin{array}{l}0,978 \\
(0,016) \\
\end{array}$ & $\begin{array}{l}0,996 \\
(0,010) \\
\end{array}$ \\
\hline Hochqualifizierten-Beschäftigtenquote & $\begin{array}{l}1,044 \\
(0,027)\end{array}$ & $\begin{array}{l}1,013 \\
(0,015)\end{array}$ & $\begin{array}{l}1,038 \\
(0,029)\end{array}$ & $\begin{array}{l}1,011 \\
(0,017)\end{array}$ \\
\hline BIP je Einwohner & $\begin{array}{l}0,998 \\
(0,008) \\
\end{array}$ & $\begin{array}{l}0,998 \\
(0,005) \\
\end{array}$ & $\begin{array}{l}1,000 \\
(0,008) \\
\end{array}$ & $\begin{array}{l}0,998 \\
(0,005) \\
\end{array}$ \\
\hline Beobachtungen & \multicolumn{2}{|c|}{118,136} & \multicolumn{2}{|c|}{118,136} \\
\hline Wald-Chi2 & \multicolumn{2}{|c|}{$72.452,02$} & \multicolumn{2}{|c|}{$141.470,21$} \\
\hline Prob> Chi2 & \multicolumn{2}{|c|}{0.0000} & \multicolumn{2}{|c|}{0.0000} \\
\hline Pseudo R2 & \multicolumn{2}{|c|}{0,1052} & \multicolumn{2}{|c|}{0,1050} \\
\hline Log-Likelihood (final value) & \multicolumn{2}{|c|}{$-109.667,16$} & \multicolumn{2}{|c|}{$-109.703,29$} \\
\hline
\end{tabular}

***/**/* bezeichnet die statistische Signifikanz auf dem $0,001 / 0,01 / 0,05$ Niveau

multinominales Regressionsmodell mit Angabe von Relativ-Risk-Ratios, clusterrobuste Standardfehler auf Ebene der Raumordnungsregionen;
Kontrolliert ist zudem das Arbeitnehmerentgelt der jeweiligen Raumordnungsregion und die konjunkturelle Gesamtlage mittels Jahresdummies;

Quelle: LIAB (LM 9310), eigene Berechnungen 


\section{Literaturverzeichnis}

\section{Literaturverzeichnis}

Abraham, Martin, und Thomas Hinz (Hg.), 2008: Arbeitsmarktsoziologie. Probleme, Theorien, empirische Befunde. Wiesbaden: VS Verlag.

Acemoglu, Daron, 1999: Changes in Unemployment and Wage Inequality: An Alternative Theory and Some Evidence. American Economic Review 89 (5): 1259-1278.

Acemoglu, Daron, 2002: Technical Change, Inequality, and The Labor Market. Journal of Economic Literature 40 (1): 7-72.

Albrech, Joachim, Philipp Fink und Heinrich Tiemann, 2016: Ungleiches Deutschland: Sozioökonomischer Disparitätenbericht 2015. Bonn: Friedrich-Ebert-Stiftung.

Alecke, Björn, und Gerhard Untiedt, 2000: Determinanten der Binnenwanderung in Deutschland seit der Wiedervereinigung. Eine makroökonometrische Analyse mit Paneldaten für die Bundesländer und den Zeitraum 1991 bis 1997. Münster. Volkswirtschaftliche Diskussionsbeiträge 309.

Arntz, Melanie, 2010: What Attracts Human Capital? Understanding the Skill Composition of Interregional Job Matches in Germany. Regional Studies 44, 4: 423-441.

Arntz, Melanie, Terry Gregory und Florian Lehmer, 2014: Can Regional Employment Disparities Explain the Allocation of Human Capital Across Space? Regional Studies 48: 1719-1738.

Arrow, Kenneth, 1971: The theory of discrimination. Princeton University. Working Paper Industrial Relations Section 30A.

Arulampalam, Wiji, 2001: Is Unemployment Really Scarring? Effects of Unemployment Experiences on Wages. Economic Journal 111 (475): 585-606.

Auspurg, Katrin, und Abraham, Martin, 2007: Die Umzugsentscheidung von Paaren als Verhandlungsproblem. Eine quasiexperimentelle Überprüfung des Bargaining-Modells. Kölner Zeitschrift für Soziologie und Sozialpsychologie 59 (2): 271-293.

Bade, Klaus J., und Jochen Oltmer, 2007: Deutschland. S. 141-170 in: Klaus J. Bade, Pieter C. Emmer, Leo Lucassen und Jochen Oltmer (Hg.), Enzyklopädie Migration in Europa. Vom 17. Jahrhundert bis zur Gegenwart. Paderborn u. a.: Verlag Ferdinand Schöningh/Wilhelm Fink-Verlag/Verlag der Neuen Zürcher Zeitung.

Baron, James, und William Bielby, 1980: Bringing the firms back in: stratification, segmentation, and the organization of work. American Sociological Review 45: 737-65.

Becker, Gary, 1962: Investment in Human Capital: A Theoretical Analysis. Journal of Political Economy 70: 9-49. 


\section{Literaturverzeichnis}

Becker, Gary, 1975: Human Capital. A theoretical and empirical analysis, with special reference to education. New York: Columbia University Press.

Bender, Stefan, Dirk Konietzka und Peter Sopp, 2000: Diskontinuität im Erwerbsverlauf und betrieblicher Kontext. Kölner Zeitschrift für Soziologie und Sozialpsychologie 52: 475-499.

Blien, Uwe, und Katja Wolf, 2002: Regional development of employment in eastern Germany: an analysis with an econometric analogue to shift-share techniques. Papers in Regional Science 81: 391-414.

Blien, Uwe, Kai Kirchhof und Oliver Ludewig, 2006: Agglomeration effects on labour demand. IAB-Discussion Paper.

Blossfeld, Hans-Peter, 1985: Berufseintritt und Berufsverlauf. Eine Kohortenanalyse über die Bedeutung des ersten Berufs in der Erwerbsbiographie. Mitteilungen aus der Arbeitsmarktund Berufsforschung 18: 177-197.

Blossfeld, Hans-Peter, und Johannes Huinink, 2001: Lebensverlaufsforschung als sozialwissenschaftliche Forschungsperspektive. Themen, Konzepte, Methoden und Probleme. BIOS 14 (2): 5-31.

Blossfeld, Hans-Peter, Melinda Mills und Fabrizio Bernardi (Hg.), 2006: Globalization, uncertainty and Men's Careers: An International Comparison. Cheltenham and Northampton: Edward Elgar.

Bohler, Karl Friedrich, und Bruno Hildenbrand, 2006: Nord - Süd. S. 324-255 in: Stephan Lessenich und Frank Nullmeier (Hg.), Deutschland - eine gespaltene Gesellschaft. Frankfurt a.M.: Campus.

Boockmann, Bernhard, und Susanne Steffes, 2005: Individual and Plant-level Determinants of Job Durations in Germany. ZEW-Discussion Paper 89.

Boockmann, Bernhard, und Susanne Steffes, 2010: Workers, firms or institutions: What determines job duration for male employees in Germany? Industrial and Labor Relations Review 64: 109-127.

Bourdieu, Pierre, 1991: Physischer, sozialer und angeeigneter physischer Raum. S. 25-34 in: Martin Wentz (Hg.), Stadt-Räume. Frankfurt am Main / New York: Campus.

Boyle, Paul, Keith Halfaree und Vaughan Robinson, 1998: Exploring contemporary migration. Harlow, Essex: Longmann.

Bresnahan, Timothy F., Erik Brynjolfsson und Lorin M. Hitt, 2002: Information Technology, Workplace Organization, and the Demand for Skilled Labor. Firm-Level Evidence. The Quarterly Journal of Economics 117 (1): 339-376. 


\section{Literaturverzeichnis}

Buch, Tanja, 2006: Regionale Mobilität auf dem deutschen Arbeitsmarkt. Eine theoretische und empirische Analyse regionaler Mismatcharbeitslosigkeit in Zeiten der Hartz-Reformen. Hamburg. Schriftenreihe Socialia 77.

Buch, Tanja, Silke Hamann, Annekatrin Niebuhr und Anja Rossen, 2014: How to Woo the Smart Ones? Evaluating the Determinants that Particularly Attract Highly Qualified People to Cities. HWWI Research Paper. Hamburg Institute of International Economics.

Buchholz, Sandra, 2008: Die Flexibilisierung des Erwerbsverlaufs. Wiesbaden: VS Verlag.

Buchholz, Sandra, und Daniela Grunow, 2006: Women's Employment in West Germany. S.6183 in: Hans-Peter Blossfeld und Heather Hofmeister (Hg.), Globalization, Uncertainty and Women's Careers. An International Comparison. Cheltenham/Northampton, MA: Edward Elgar.

Bundesinstitut für Bau-, Stadt- und Raumforschung, 2016: Laufende Raumbeobachtung Raumabgrenzungen.

http://www.bbsr.bund.de/BBSR/DE/Raumbeobachtung/Raumabgrenzungen/Raumordnun gsregionen/raumordnungsregionen_node.html [06.02.2019].

Coleman, James S., 1990: Foundations of Social Theory. Cambridge: Harvard University Press.

Coleman, James S., 1991: Grundlagen der Sozialtheorie. Band 1: Handlungen und Handlungssysteme. München: Oldenbourg.

Damelang, Andreas, 2007: Räumliche Mobilität von türkischen Arbeitnehmern. Eine Analyse mit der IAB-Beschäftigtenstichprobe 2001. IAB-Discussion Paper.

De Jong, Gordon, und James Fawcett, 1981: Motivations for Migration: An Assessment and Value-Expectancy Research Modell. S. 13-58 in: Gordon De Jong und Robert W. Gardener (Hg.), Migration decision Making. Multidisciplinary Approaches to Microlevel Studies in Developed and Developing Countries. New York: Pergamon Press.

Devereux, Paul, und Robert Hart, 2006: Real Wage Cyclicality of Job Stayers, Within-Company Job Movers, and Between-Company Job Movers. Industrial and Labor Relations Review 60: $105-119$.

Doeringer, Peter, und Michael Piore, 1971: Internal labor markets and manpower analysis. Lexington: D.C. Heath.

Doering-Manteuffel, Anselm, 2007: Nach dem Boom. Brüche und Kontinuitäten der Industriemoderne seit 1970. Vierteljahrshefte für Zeitgeschichte 55 (4): 559-581.

Doering-Manteuffel, Anselm, und Lutz Raphael, 2011: Der Epochenbruch in den 1970er-Jahren. Thesen zur Phänomenologie und den Wirkungen des Strukturwandels »nach dem Boom«. S. 25-40 in: Knud Andresen, Ursula Bitzegeio und Jürgen Mittag (Hg.), Nach dem Strukturbruch? Kontinuität und Wandel von Arbeitsbeziehungen und Arbeitswelt(en) seit den 1970er-Jahren. Bonn: J.H.W. Dietz Nachf.. 
Dombois, Rainer, 1999: Der schwierige Abschied vom Normalarbeitsverhältnis. Aus Politik und Zeitgeschichte 37: 13-20.

Dörre, Klaus, 2005a: Prekäre Beschäftigung - ein unterschätztes Phänomen in der Debatte um Marktsteuerung und Subjektivierung von Arbeit. S. 180-206 in: Karin Lohr und Hildegard Nickel (Hg.), Subjektivierung von Arbeit - Riskante Chancen. Münster: westfälisches Dampfboot.

Dörre, Klaus, 2005b: Prekarität - Eine arbeitspolitische Herausforderung. WSI-Mitteilungen 58: 250-258.

Duranton, Gilles, 2004: The economics of production systems: Segmentation and skill-biased change. European Economic Review 48 (2): 307-336.

Dütsch, Matthias, Verena Liebig und Olaf Struck, 2013: Erosion oder Stabilität berufsfachlicher Qualifikationen? Eine Analyse der Entwicklung und Determinanten beruflicher Mobilität. Kölner Zeitschrift für Soziologie und Sozialpsychologie 65 (3): 505-531.

Dütsch, Matthias, und Olaf Struck, 2014: Employment trajectories in Germany. Do firm characteristics and regional disparities matter? Journal for Labour Market Research 47 (12): 107-127.

Dütsch, Matthias, Franziska Ganesch und Olaf Struck, 2019: Employment trajectories in heterogeneous regions: Evidence from Germany. Advances in Life Course Research 40: 4384.

Dorn, Raphael Emanuel, 2018: Alle in Bewegung. Räumliche Mobilität in der Bundesrepublik Deutschland 1980-2010. Göttingen: Vandenhoeck \& Ruprecht.

Engelhardt, Henriette, 2009: Zum Wandel der Korrelation von Fertilität und Frauenerwerbstätigkeit in Raum und Zeit: Eine empirische Analyse unter Berücksichtigung der Effekteausgewählter sozialer Indikatoren. S. 245-263 in: Henriette Engelhardt, (Hg.), Schwerpunktthemaheft Fertilität und Frauenerwerbstätigkeit. Zeitschrift für Familienforschung 21(3).

Eichhorst, Werner, Gesine Stephan und Olaf Struck, 2017: Struktur und Ausgleich des Arbeitsmarktes. Working paper Forschungsförderung, Hans-Böckler-Stiftung Nr. 28.

Erlinghagen, Marcel, 2005: Entlassungen und Beschäftigungssicherheit im Zeitverlauf. Zur Entwicklung unfreiwilliger Arbeitsmarktmobilität in Deutschland. Zeitschrift für Soziologie 34: $147-168$.

Esser, Hartmut, 1996: Soziologie: Allgemeine Grundlagen. Frankfurt am Main: Campus.

Evertsson, Marie, und Daniela Grunow, 2012: Women's work interruptions and career prospects in Germany and Sweden? International Journal of Sociology and Social Policy 32 (9): 561-575.

Fabian, Gregor, und Karl-Heinz Minks, 2008: Muss i denn zum Städtele hinaus? Erwerbsmobilität von Hochschulabsolventen. HIS-Magazin 3: 4-5. 
Farhauer, Oliver, und Nadia Granato, 2006: Regionale Arbeitsmärkte in Westdeutschland: Standortfaktoren und Branchenmix entscheidend für Beschäftigung. IAB-Kurzbericht 4.

Fassmann, Heinz, und Peter Meusburger, 1997: Arbeitsmarktgeographie. Erwerbstätigkeit und Arbeitslosigkeit im räumlichen Kontext. Stuttgart: Teubner.

Fink, Philipp, und Heinrich Tiemann, 2016: Deutschland driftet auseinander. S. 3-5 in: Joachim Albrech, Philipp Fink und Heinrich Tiemann (Hg.), Ungleiches Deutschland: Sozioökonomischer Disparitätenbericht 2015. Bonn: Friedrich-Ebert-Stiftung.

Fink, Philipp, Martin Hennicke und Heinrich Tiemann, 2019: Ungleiches Deutschland Sozioökonomischer Disparitätenbericht 2019. Bonn: Friedrich-Ebert-Stiftung. http://library.fes.de/pdf-files/fes/15400-20190528.pdf [16.10.2019].

Fitzenberger, Bernd, und Alfred Garloff, 2007: Labor market transitions and the wage structure in Germany. Jahrbücher für Nationalökonomie und Statistik 227: 115-152.

Friedman, Debra, und Michael Hechter, 1988: The Contribution of Rational Choice Theory to Macro-sociological Research. Sociological Theory 6: 201-218.

Fuchs, Michaela, 2010: Determinanten regionaler Beschäftigungsentwicklung. Nürnberg: Institut für Arbeitsmarkt- und Berufsforschung.

Fujita, Masahisa, Paul Krugman und Anthony Venables, 2001: The spatial economy. Cities, regions and international trade. Cambridge: MIT Press.

Ganesch, Franziska, 2018: Regional Mobility and Spatial Inequality: Determinants of Spatial Labor Market Behavior Considering Firm- and Region-Specific Factors. Spatial Research and Planning 76: 497-514.

Ganesch, Franziska, Matthias Dütsch und Olaf Struck, 2019: Regionale Mobilität am Arbeitsmarkt. Individuelle, betriebliche und wirtschaftsstrukturelle Determinanten von Mobilität und Einkommen. Kölner Zeitschrift für Soziologie und Sozialpsychologie 71 (2): 181-210.

Ganesch, Franziska, Matthias Dütsch und Olaf Struck, 2020: Arbeitsplatzmobilität zwischen Ost-, Nord-, und Süddeutschland. Erfolgsfaktoren von Einkommenszuwächsen. Sozialer Fortschritt, im Erscheinen.

Gerlach, Knut, Wolfgang Meyer und Georgi Tsertsvadze, 2002: Entwicklung der qualifikatorischen Segregation im Verarbeitenden Gewerbe. S. 51-84 in: Lutz Bellmann, und Arne Kölling (Hg.), Betrieblicher Wandel und Fachkräftebedarf. Nürnberg: Institut für Arbeitsmarkt- und Berufsforschung.

Gerstenberg, Susanne, 2014: Flexible Beschäftigung und Räumliche Mobilität im betrieblichen Kontext: Mixed-Methods-Analysen betrieblicher Mobilitätspolitik. Dissertation, FriedrichSchiller-Universität Jena. 


\section{Literaturverzeichnis}

Giddens, Anthony, 1991: The Consequences of Modernity. Cambridge: Polity Press.

Giesecke, Johannes, und Jan Paul Heisig, 2010: Destabilisierung und Destandardisierung, aber für wen? Die Entwicklung der westdeutschen Arbeitsplatzmobilität seit 1984. Kölner Zeitschrift für Soziologie und Sozialpsychologie 62: 403-435.

Glaeser, Edward, und David Maré, 2001: Cities and Skills. Journal of Labor Economics 19 (2): 316-342.

Glorius, Birgit, 2010: Go West: Internal migration in Germany after reunification. Belgeo 3: 281292.

Granato, Nadia, Anette Haas, Silke Hamann und Annekatrin Niebuhr, 2009: Arbeitskräftemobilität in Deutschland - Qualifikationsspezifische Befunde regionaler Wanderungs- und Pendlerströme. Raumforschung und Raumordnung 67: 21-33.

Grotheer, Michael, Olaf Struck, Lutz Bellmann und Tilo Gewiese, 2004: Determinanten von Beschäftigungsstabilität. Chancen und Risiken von 'Entrants' im ost-westdeutschen Vergleich. S. 125-156 in: Olaf Struck und Christoph Köhler (Hg.), Beschäftigungsstabilität im Wandel? Mering: Rainer Hampp Verlag.

Haas, Anette, 2000: Arbeitsmarktausgleich: Regionale Mobilität gestiegen. Bei einem Betriebswechsel werden immer öfter größere Entfernungen überwunden - gerade auch von Arbeitslosen. IAB-Kurzbericht 4.

Hacket, Anne, 2009: Lohnt sich Mobilität? Einkommensperspektiven in internen und externen Arbeitsmärkten in den ersten Berufsjahren. Wiesbaden: VS Verlag.

Hausmann, Ann-Christin, und Corinna Kleinert, 2014: Berufliche Segregation auf dem Arbeitsmarkt: Männer- und Frauendomänen kaum verändert. IAB-Kurzbericht, Institut für Arbeitsmarkt- und Berufsforschung (IAB) der Bundesagentur für Arbeit, 9: 1-8.

Hart, Robert, 2006: Worker-Job Matches, Job Mobility and Real Wage Cyclicality. Economica 73: 287-298.

Heinz, Walter R., 1996: Status passages as micro-macro linkages in life course research. S. 51-65 in: Walter R. Heinz und Ansgar Weymann (Hg.), Society and biography: interrelationships between social structure, institutions and the life course. Weinheim: Deutscher Studienverlag.

Hendry, Chris, 2003: Applying employment systems theory to the analysis of national models of HRM. The International Journal of Human Resource Management 14: 1430-1442.

Hillmert, Steffen, 2006: Mobilität am Arbeitsmarkt und regionale Disparitäten. S. 3391-3401 in: Karl-Siegbert Rehberg (Hg.), Soziale Ungleichheit, kulturelle Unterschiede: Verhandlungen des 32. Kongresses der Deutschen Gesellschaft für Soziologie in München. Teilb. 1 und 2, Frankfurt: Campus. 


\section{Literaturverzeichnis}

Hinz, Thomas, und Hermann Gartner, 2005: Geschlechtsspezifische Lohnunterschiede in Branchen, Berufen und Betrieben. Zeitschrift für Soziologie, 34: 22-39.

Hinz, Thomas, und Martin Abraham, 2008: Theorien des Arbeitsmarktes: Ein Überblick. S. 17-68 in: Martin Abraham und Thomas Hinz (Hg.), Arbeitsmarktsoziologie. Probleme, Theorien, empirische Befunde. Wiesbaden: VS Verlag.

Hirschman, Albert, 1958: Strategy of Economic Development. New Haven: Yale University Press.

Huber, Franz, 2009: Social Capital of Economic Clusters: Towards a Network-Based Conception of Social Resources. Tijdschrift voor economische en sociale geografie 100 (2): 160-170.

Hübler, Olaf, und Thomas Walter, 2009: Beschäftigungsstabilität im Konjunkturzyklus - Eine empirische Analyse mit Individualdaten für Westdeutschland 1984-2005. Schmollers Jahrbuch 129: 1-35.

Huinink, Johannes, und Stefanie Kley, 2008: Regionaler Kontext und Migrationsentscheidungen im Lebensverlauf. Kölner Zeitschrift für Soziologie und Sozialpsychologie 48 (8): 163-184.

Jacob, Marita, und Corinna Kleinert, 2014: Marriage, Gender, and Class: The Effect of Partner Resources on Unemployment Exit in Germany. Social Forces 92 (3): 839-871.

Jacobebbinghaus, Peter, 2008: LIAB-Datenhandbuch. Version 3.0. FDZ-Datenreport 3: Bundesagentur für Arbeit.

Johnson, William R., 1978: A Theory of Job Shopping. Quarterly Journal of Economics 97: 261277.

Jovanovic, Boyan, und Rafael Rob, 1989: The growth and diffusion of knowledge. The Review of Economic Studies, 56 (4): 569-582.

Kalter, Frank, 1994: Pendeln statt Migration? Die Wahl und Stabilität von Wohnort-ArbeitsortKombinationen. Zeitschrift für Soziologie 23: 460-476.

Kalter, Frank, 1997: Wohnortwechsel in Deutschland. Ein Beitrag zur Migrationstheorie und zur empirischen Anwendung von Rational-Choice-Modellen. Opladen: Leske + Budrich.

Kawka, Rupert, 2009: Regionaler Preisindex. Berichte / Bundesamt für Bauwesen und Raumordnung. BBSR 30.

Kelle, Markus, 2016: Wage Growth, Urbanization, and Firm Characteristics - Evidence for Germany. Ruhr Economic Papers 631.

Kley, Stefanie A., 2009: Migration im Lebensverlauf. Der Einfluss von Lebensbedingungen und Lebenslaufereignissen auf den Wohnortwechsel. Wiesbaden: VS Verlag. 


\section{Literaturverzeichnis}

Kley, Stefanie A., und Clara H. Mulder, 2010: Considering, planning, and realizing migration in early adulthood. The influence of life-course events and perceived opportunities on leaving the city in Germany. Journal of Housing and the Built Environment 25: 73-94.

Kley, Stefanie A., 2016. Räumliche Mobilität. S. 481-500 in: Yasemin Niephaus, Michaela Kreyenfeld und Reinhold Sackmann (Hg.), Handbuch Bevölkerungssoziologie. Wiesbaden: VS Verlag.

Kogan, Irena, 2004: Labour market careers of immigrants in Germany and Great Britain. Journal of International Migration and Integration, 5 (4): 419-449.

Kogan, Irena, 2011: The price of being an outsider: Labour market flexibility and immigrants' employment paths in Germany. International Journal of Comparative Sociology 52 (4): 264283.

Köhler, Christoph, Olaf Struck, Michael Grotheer, Alexandra Krause, Ina Krause und Tim Schröder, 2008: Offene und geschlossene Beschäftigungssysteme. Determinanten, Risiken und Nebenwirkungen. Wiesbaden: VS Verlag.

Kohli, Martin, 1985: Die Institutionalisierung des Lebenslaufs. Historische Befunde und theoretische Argumente. Kölner Zeitschrift für Soziologie und Sozialpsychologie 37 (1): 129.

Kratz, Fabian, und Josef Brüderl, 2013: Returns to Regional Migration: Causal Effect or Selection on Wage Growth? Schmollers Jahrbuch 133: 227-238.

Kreckel, Reinhard, 1983: Soziale Ungleichheit und Arbeitsmarktsegmentierung. S. 137-162 in: Reinhard Kreckel (Hg.), Soziale Ungleichheit (Soziale Welt; Sonderband 2), Göttingen: Schwartz.

Krugman, Paul, 1991: Geography and trade. Cambridge: MIT Press.

Krugman, Paul, 1998: What's new about the new economic geography? Oxford Review of Economic Policy 14: 7-17.

Lazear, Edward P., 1981: Agency, earning profiles, productivity and hours restrictions. American Economic Review 71: 606-620.

Lee, Everett S., 1972: Eine Theorie der Wanderung. S. 115-129 in: György Széll (Hg.), Regionale Mobilität. Elf Aufsätze. Nymphenburger Texte zur Wissenschaft, Bd. 10, München: Nymphenburger Verlagshandlung.

Lehmer, Florian, und Joachim Möller, 2008: Group-specific effects of interregional mobility on earnings - a microdata analysis for Germany. Regional Studies 42: 657-673.

Lehmer, Florian, und Johannes Ludsteck, 2011: The returns to job mobility and inter-regional migration: Evidence from Germany. Papers in Regional Science 90: 549-572. 


\section{Literaturverzeichnis}

Leitner, Sigrid, Ilona Ostner und Margit Schratzenstaller (Hg.), 2004: Wohlfahrtsstaat und Geschlechterverhältnis im Umbruch: Was kommt nach dem Ernährermodell? Wiesbaden: VS Verlag.

Lucas, Robert, 1988: On the mechanics of economic development. Journal of Monetary Economics 22: 3-42.

Maier, Michael F., und Maresa Sprietsma, 2016: Does it pay to move? Returns to regional mobility at the start of the career for tertiary education graduates. ZEW Discussion Paper 16-060. http://ftp.zew.de/pub/zew-docs/dp/dp16060.pdf [03.11.2019].

Manzoni, Anna, 2012: In and out of employment: Effects in panel and life-history data. Advances in Life Course Research 17: 11-24.

Mayer, Karl U. (Hg.), 1990: Lebensverläufe und sozialer Wandel. Opladen: Westdeutscher Verlag.

Mayer, Karl U., 2009: New directions in life course research. Annual Review of Sociology 35: 413-433.

Mayer, Karl U., und Hans-Peter Blossfeld, 1990: Die gesellschaftliche Konstruktion sozialer Ungleichheit im Lebensverlauf. S. 297-318 in: Peter Berger und Stefan Hradil (Hg.), Lebenslagen, Lebensläufe, Lebensstile. Göttingen: Schwartz.

McKenna, Christopher J., 1985: Uncertainty and the labour market: Recent developments in jobsearch theory. Brighton, Sussex.

Mertens, Antje, und Anette Haas, 2006: Regionale Arbeitslosigkeit und Arbeitsplatzwechsel in Deutschland - Eine Analyse auf Kreisebene. Jahrbuch für Regionalwissenschaft 26: 147169.

Meusburger, Peter, 1998: Bildungsgeographie. Wissen und Ausbildung in der räumlichen Dimension. Heidelberg: Spektrum.

Mincer, Jacob, 1958: Investment in Human Capital and Personal Income Distribution. Journal of Political Economy 66: 281-302.

Niedomysl, Thomas, und Høgni Kalsø Hansen, 2010: What matters more for the decision to move: jobs versus amenities. Environment and Planning A 42 (7): 1636-1649.

Nisic, Natascha, 2010: Mitgegangen - mitgefangen? Die Folgen von Haushaltsumzügen für die Einkommenssituation von Frauen in Partnerschaften. Kölner Zeitschrift für Soziologie und Sozialpsychologie 62 (3): 515-549.

Nisic, Natascha, 2011: Determinanten und Konsequenzen beruflich bedingter räumlicher Mobilität im Kontext von Partnerschaft und Haushalt. Dissertation. Elektronische Publikation an der Friedrich-Alexander Universität Nürnberg-Erlangen. 
Nisic, Natascha, und Martin Abraham, 2014: Familie und Mobilität. S. 675-708 in: Paul B. Hill und Johannes Kopp (Hg.), Handbuch der Familiensoziologie. Wiesbaden: VS Verlag.

Novemsky, Nathan, und Daniel Kahneman, 2005: The Boundaries of Loss Aversion. Journal of Marketing Research 42 (2): 119-128.

Phelps, Edmund, 1972: The Statistical Theory of Racism and Sexism. American Economic Review 62 (4): 659-661.

Poelhekke, Steven, 2013: Human Capital and Employment Growth in German Metropolitan Areas: New Evidence. Regional Studies 47 (2): 245-263.

Porter, Michael E., 2000: Location, Competition, and Economic Development: Local Clusters in a Global Economy. Economic Development Quarterly 14 (1): 15-34.

Ravenstein, Ernest G., 1972: Die Gesetze der Wanderung I und II. S.41-64 in: György Széll (Hg.), Regionale Mobilität. München: Nymphenburger Verlagshandlung.

Reichelt, Malte, und Martin Abraham, 2015: Occupational and regional mobility as substitutes. A new approach to understanding job changes and wage inequality. IAB-Discussion Paper 127.

Rendtel, Ulrich, und Johannes Schwarze, 1996: Schätzungen von Lohnkurven für Westdeutschland mit einem verallgemeinerten Varianz-Komponenten-Modell. Mitteilungen aus der Arbeitsmarkt- und Berufsforschung 29: 491-493.

Rolfes, Manfred, 1996: Regionale Mobilität und akademischer Arbeitsmarkt. Osnabrück: Rasch.

Romer, Paul, 1986: Increasing Returns and Long Run Growth. Journal of Political Economy 94: 1002-1037.

Romer, Paul, 1990: Endogenous technological change. Journal of Political Economy 98 (5): 71102.

Ruppenthal, Silvia, 2010: Vielfalt und Verbreitung berufsbedingter räumlicher Mobilität im europäischen Vergleich. Mitteilungen aus dem Bundesinstitut für Bevölkerungsforschung 31: 2-7.

Ryder, Norman B., 1965: The Cohort as a Concept in the Study of Social Change. American Sociological Review 30 (6): 843-861.

Sander, Nikola, 2014: Internal Migration in Germany, 1995-2010: New Insights into East-West Migration and Reurbanisation. Comparative Population Studies 39, 2: 217-246.

Schlitte, Friso, 2012: Local human capital, segregation by skill, and skill-specific employment growth. Papers in Regional Science 91 (1): 85-106. 
Schlitte, Friso, Fabian Böttcher, Annektrin Niebuhr und Javier Diez, 2010: The determinants of regional disparities in skill segregation - Evidence from a cross section of German regions. HWWI Research Paper: 1-36.

Schlömer, Claus, 2004: Binnenwanderungen seit der deutschen Einigung. Raumforschung und Raumordnung 62, 2: 96-108.

Schneider, Norbert F., Ruth Limmer und Kerstin Ruckdeschel, 2002a: Mobil, flexibel, gebunden. Familie und Beruf in der mobilen Gesellschaft. Frankfurt/Main: Campus-Verlag.

Schneider, Norbert F., Ruth Limmer und Kerstin Ruckdeschel, 2002b: Berufsmobilität und Lebensform. Sind berufliche Mobilitätserfordernisse in Zeiten von Globalisierung noch mit Familie vereinbar? Stuttgart. Schriftenreihe des Bundesministeriums für Familien, Senioren, Frauen und Jugend 208.

Schneider, Norbert F., Silvia Ruppenthal und Detlev Lück, 2009: Beruf, Mobilität und Familie. S. 111-136 in: Günter Burkart (Hg.), Zukunft der Familie. Prognosen und Szenarien. Zeitschrift für Familienforschung Sonderheft Band 6, Opladen: Budrich.

Schneider, Norbert F., Heiko Rüger und Silvia Ruppenthal, 2016: Mobilität und mobile Lebensformen. S. 501-525 in: Yasemin Niephaus, Michaela Kreyenfeld und Reinhold Sackmann (Hg.), Handbuch Bevölkerungssoziologie. Wiesbaden: VS Springer.

Schroer, Markus, 2006: Raum, Macht und soziale Ungleichheit: Pierre Bourdieus Beitrag zu einer Soziologie des Raums. Leviathan 34: 105-123.

Seibert, Holger, 2008: Räumliche Mobilität als Notwendigkeit zur Vermeidung von Arbeitslosigkeit am Beispiel der Region Berlin-Brandenburg. S. 37-46 in: Eva Barlösius und Claudia Neu (Hg.), Peripherisierung - eine neue Form sozialer Ungleichheit? Berlin: Berlin-Brandenburgische Akademie der Wissenschaften.

Sengenberger, Werner, 1987: Struktur und Funktionsweise von Arbeitsmärkten. Die Bundesrepublik Deutschland im internationalen Vergleich. Frankfurt am Main: Campus.

Sennett, Richard, 1998: The Corrosion of Character. The Personal Consequences of Work in the New Capitalism, New York / London: W. W. Norton.

Sennett, Richard, 2006: Der flexible Mensch. Die Kultur des neuen Kapitalismus, aus dem Englischen von Martin Richter. Berlin: Berliner Taschenbuch-Verlag.

Seynstahl, Christian, 2015: Die Arbeitsortmobilität hochqualifizierter Beschäftigter. Eine arbeitsmarktgeographische Analyse und Modellierung am Beispiel Mainfranken. Mannheim: MetaGIS Systems.

Shapiro, Jesse, 2006: Smart Cities: Quality of Life, Productivity, and the Growth Effects of Human Capital. The Review of Economics and Statistics 88: 324-335. 


\section{Literaturverzeichnis}

Sixtus, Frederick, Manuel Slupina, Sabine Sütterlin, Julia Amberger und Reiner Klingholz, 2019: Teilhabeatlas: Ungleichwertige Lebensverhältnisse und wie die Menschen sie wahrnehmen. https://www.berlin-institut.org/fileadmin/user_upload/Teilhabeatlas/Teilhabe_Online.pdf [06.09.2019].

Sjaastad, Larry, 1962: The Costs and Returns of Human Migration. Journal of Political Economy 70: 80-93.

Slupina, Manuel, Theresa Damm und Reiner Klingholz, 2015: Im Osten auf Wanderschaft. Wie Umzüge die demografische Landkarte zwischen Rügen und Erzgebirge verändern. Berlin.

Spellerberg, Anette, 2016: Regionale Disparitäten. S. 350-359 in: Datenreport 2016. Ein Sozialbericht für die Bundesrepublik Deutschland, Statistisches Bundesamt (Hg.), Wissenschaftszentrum Berlin für Sozialforschung, Bonn: Bundeszentrale für politische Bildung.

Spence, Michael, 1973: Job marketing signaling. Quarterly Journal of Economics 87 (3): 355-374.

Spence, Michael, 2002: Signaling in retrospect and the informational structure of markets. American Economic Review 92 (3): 434-459.

Statistisches Bundesamt, 2016: Datenreport 2016: Ein Sozialbericht für die Bundesrepublik Deutschland. Bonn: Bundeszentrale für politische Bildung.

Statistisches Bundesamt, 2017: Verdienste auf einen Blick, https://www.destatis.de/DE/Publikationen/Thematisch/VerdiensteArbeitskosten/Arbeitneh merverdienste/BroschuereVerdiensteBlick0160013179004.pdf [06.11.2019].

Struck, Olaf, 2006: Flexibilität und Sicherheit: Empirische Befunde, theoretische Konzepte und institutionelle Gestaltung von Beschäftigungsstabilität. Wiesbaden: VS Verlag.

Struck, Olaf, Michael Grotheer, Tim Schröder und Christoph Köhler, 2007: Instabile Beschäftigung: Neue Ergebnisse zu einer alten Kontroverse. Kölner Zeitschrift für Soziologie und Sozialpsychologie 59 (2): 294-317.

Struck, Olaf, und Matthias Dütsch, 2012: Gesicherte Mobilität am Arbeitsmarkt. Zur Bedeutung berufsfachlicher Qualifikationen in geschlossenen und offenen Beschäftigungssystemen. Industrielle Beziehungen 19: 124-153.

Struck, Olaf, 2017: Aufschwung und Unzufriedenheit. Strukturwandel und Lebenssituation in Ostdeutschland. Bamberg. Universität Bamberg, Professur für Arbeitswissenschaft. Working Paper 19.

Südekum, Jens, 2008: Convergence of the skill composition across German regions. Regional Science and Urban Economics 38: 148-159. 


\section{Literaturverzeichnis}

Tully, Claus J., 2006: Mobiler Alltag. Mobilität zwischen Option und Zwang, Vom Zusammenspiel biographischer Motive und sozialer Vorgaben. Wiesbaden: Springer VS.

Urry, John, 2007: Mobilities. Cambridge, Malden: Polity Press.

Vobruba, Georg, 1990: Lohnarbeitszentrierte Sozialpolitik in der Krise der Lohnarbeit. S. 11-80 in: Georg Vobruba (Hg.), Strukturwandel der Sozialpolitik. Lohnarbeitszentrierte Sozialpolitik und soziale Grundsicherung. Frankfurt a.M.: Suhrkamp.

Vobruba, Georg, 2000: Alternativen zur Vollbeschäftigung. Die Transformation von Arbeit und Einkommen. Frankfurt: Suhrkamp.

Wagner, Michael, 1989: Räumliche Mobilität im Lebensverlauf. Eine empirische Untersuchung sozialer Bedingungen der Migration. Stuttgart: Enke.

Wheeler, Christopher, 2006: Cities and the growth of wages among young workers: Evidence from the NLSY. Journal of Urban Economics 60: 162-184.

Windzio, Michael, 2004a: Kann der regionale Kontext zur „Arbeitslosenfalle“ werden? Der Einfluss der Arbeitslosigkeit auf die Mobilität zwischen regionalen Arbeitsmärkten in Westdeutschland. Kölner Zeitschrift für Soziologie und Sozialpsychologie 56 (2): 257-278.

Windzio, Michael, 2004b: Zwischen Nord- und Süddeutschland: Die Überwindung räumlicher Distanzen bei der Arbeitsmarktmobilität. Zeitschrift für ArbeitsmarktForschung 37: 29-44.

Windzio, Michael, 2013: Räumliche Mobilität. S. 664-675 in: Steffen Mau und Nadine M. Schöneck (Hg.), Handwörterbuch zur Gesellschaft Deutschlands. Wiesbaden: VS Verlag.

Windzio, Michael, und Johannes Huinink, 2010: Migration, regionale Entwicklung und die Integration von Zuwanderern. Eine kurze Einführung. S. 9-19 in: Wolfgang-Ritter-Stiftung (Hg.), Migration und regionale Entwicklung. Oldenburg: Isensee.

Wirtschafts- und Sozialwissenschaftliches Institut der Hans-Böckler-Stiftung, 2018: Lohnspiegel, https://www.lohnspiegel.de/html/index.php [01.03.2018].

Wolpert, Julian, 1965: Behavioral aspects of the decision to migrate. Papers in Regional Science 15: 159-169. 
https://doi.org/10.1016/j.alcr.2019.03.002

Dütsch, Matthias, Franziska Ganesch und Olaf Struck, 2019: Employment trajectories in heterogeneous regions: Evidence from Germany. Advances in Life Course Research 40: 43-84.

\begin{abstract}
A B S T R A C T
To what extent do regional characteristics influence employment trajectories? Do regional factors diversely affect the employment careers of different sociodemographic groups? By investigating these questions, we extend current life course research in two ways: First, from a conceptual perspective, we use approaches from regional economics in addition to established sociological labour market theories to gain insights into the effects of regional determinants on individual labour market outcomes. Second, from a methodological point of view, we conduct event history analyses based on a German dataset that contains information on individuals, firms and regions. Our results show that there are considerable regional heterogeneities regarding population density and the amount of human capital endowment, both of which influence working careers differently. Regional agglomeration predominantly offers opportunities in terms of employment trajectories, while regional human capital accumulation increases employment risks. Additionally, our findings indicate that group-specific inequalities with respect to employment careers can be weakened or even strengthened by regional frame conditions. Female and foreign employees benefit most from denser regions and from a higher human capital endowment. By contrast, the unemployment risks of workers who previously experienced unemployment periods during their working lives are increased by both of these regional characteristics. Findings regarding education level are mixed: Workers with occupational qualifications profit from regional agglomeration to a greater extent than do low or even generally qualified workers. However, a high local human capital endowment leads to skill segregation between vocationally trained and highly qualified employees.
\end{abstract}

\title{
Keywords:
}

Regional disparities, Employment career, Life course, Germany, Event history analysis 


\section{Ganesch, Franziska, Matthias Dütsch und Olaf Struck, 2019: Regionale Mobilität am Arbeitsmarkt. Individuelle, betriebliche und wirtschaftsstrukturelle Determinanten von Mobilität und Einkommen. Kölner Zeitschrift für Soziologie und Sozialpsychologie 71 (2): 181-210.}

Zusammenfassung Untersucht wird, welche individuellen, betrieblichen und regionalen wirtschafstrukturellen Merkmale eine erfolgreiche regionale Mobilität von Vollzeiterwerbstätigen unterstützen. Zu Einkommenseffekten im Rahmen von regionaler Mobilität besteht ebenso Forschungsbedarf wie hinsichtlich des Einflusses regionaler Strukturdaten. Analysiert wird ein integrierter Betriebs- und Personendatensatz (LIAB) des Instituts für Arbeitsmarkt- und Berufsforschung, der um regionale Strukturindikatoren (INKAR) ergänzt wurde. Die Ergebnisse der binären und multinomialen logistischen Regressionsmodelle zeigen unter anderem, dass überregionale Mobilität sowie auch damit einhergehende Einkommenserfolge besonders von Individualmerkmalen, wie dem Alter und der Qualifikation, bestimmt werden. Regionale Wirtschafts- und Strukturindikatoren erweisen sich als etwas weniger bedeutsam bei Entscheidungen für regionale Mobilität und deren Erfolge. Ländliche Regionen oder Regionen mit höherer Arbeitslosigkeit werden letztlich nicht häufiger verlassen. In wirtschaftsstrukturell entwickelten Regionen findet sich gleichwohl überregionale Mobilität. In ihnen zeigt sich wider Erwarten jedoch keine höhere Wahrscheinlich- keit von Aufstiegen. In Zielregionen mit einem hohen Anteil an hochqualifizierten Beschäftigten zeigt sich für Akademiker unmittelbar und bereinigt um das regionale Preisniveau eine höhere Wahrscheinlichkeit von Einkommensverlusten. Offen bleibt, wie sich Zielbetriebskontexte im weiteren Verlauf des Verbleibs im Zielraum auf die Einkommenschancen der verschiedenen Beschäftigtengruppen auswirken.

Schlüsselwörter:

Regionale Mobilität · Arbeitsmarkt · Regionale Strukturindikatoren · Einkommen 
https://doi.org/10.1007/s13147-018-0551-2

\title{
Franziska Ganesch, 2018: Regional Mobility and Spatial Inequality: Determinants of Spatial Labor Market Behavior Considering Firm- and Region-Specific Factors. Spatial Research and Planning 76 (2018): 497- 514.
}

\begin{abstract}
Regional disparities exist between states as well as within countries. In the Federal Republic of Germany, as a result of reunification, there are still considerable regional differences in terms of economic conditions. Local economic and structural circumstances affect individual employment opportunities and can be a motive for migration. Based on rational-economic approaches, the article examines the spatial labor market behavior of full-time employees subject to social security contributions, taking into account individual, firm-specific and regional characteristics. Workplace mobility is analyzed as a job move between the macro regions of North, South and East Germany. For this purpose, a linked employer-employee dataset (LIAB) from the "Institute for Employment Research" is used, supplemented by regional structural indicators (INKAR) at the level of the spatial planning regions. The analyses using binary logistic regression models show that transregional mobility especially depends on individual characteristics such as age and qualifications. Regarding regional features, it can be seen that the emigration tendency of younger employees increases with the local level of unemployment. Overall, the results do not indicate that there is a single-track brain drain from structurally weak regions. Nevertheless, these regions face a problem in age-specific migration patterns. The outflow of young workers weakens structurally poor areas directly and permanently.
\end{abstract}

Keywords Regional mobility $\cdot$ Spatial inequality $\cdot$ Labor market $\cdot$ Regional structural indicators 


\title{
Arbeitsplatzmobilität zwischen Ost-, Nord-, und Süddeutschland: Erfolgsfaktoren von Einkommenszuwächsen
}

\author{
Franziska Ganesch, Matthias Dütsch, Olaf Struck \\ Franziska Ganesch \\ Wissenschaftliche Mitarbeiterin an der Professur für Arbeitswissenschaft an der sozial- und \\ wirtschaftswissenschaftlichen Fakultät der Otto-Friedrich-Universität Bamberg, Feldkirchenstraße 21, \\ 96045 Bamberg, Deutschland \\ E-Mail: franziska.ganesch@uni-bamberg.de \\ Matthias Dütsch \\ Wissenschaftlicher Mitarbeiter an der Geschäfts- und Informationsstelle für Mindestlohn der \\ Bundesanstalt für Arbeitsschutz und Arbeitsmedizin, Nöldnerstraße 40-42, 10317 Berlin, Deutschland \\ E-Mail: matthias.duetsch@geschaeftsstelle-mindestlohn.de

\section{Olaf Struck} \\ Professor für Arbeitswissenschaft an der sozial- und wirtschaftswissenschaftlichen Fakultät der Otto- \\ Friedrich-Universität Bamberg, Feldkirchenstraße 21, 96045 Bamberg, Deutschland \\ E-Mail: olaf.struck@uni-bamberg.de
}

In Deutschland beeinflussen regionale Disparitäten besonders auch zwischen Ost- und Westdeutschland individuelle Lebens- und Einkommenschancen. Individuen können versuchen, ihre Arbeitsbedingungen - etwa ihr Einkommen - durch räumliche Mobilität zu verbessern. Der vorliegende Beitrag untersucht Mobilität zwischen Ost-, Nord- und Süddeutschland und damit einhergehende Einkommensveränderungen. Basis ist ein Linked Employer-Employee Datensatz, der um regionale Strukturindikatoren ergänzt wurde. Die Ergebnisse zeigen: Jüngere und Hochqualifizierte wechseln häufiger und realisieren bei Betriebswechseln mit höherer Wahrscheinlichkeit Einkommenszuwächse. Anreize für OstWestmobilität bestehen fort, da bei Wechseln aus Ostdeutschland in Richtung Nord- oder Süddeutschland preisniveaubereinigt die Wahrscheinlichkeit von Einkommenszuwächsen höher ist, als bei Wechseln innerhalb Ostdeutschlands. Wechsel nach Ostdeutschland können mit Einkommensverlusten, aber auch Einkommenszuwächsen einhergehen.

\section{Workplace-mobility between East, North and South Germany. Success factors of income increases}

In Germany, regional disparities especially between East and West Germany influence individual life and income opportunities. Individuals can try to improve their working conditions - for example their income - through spatial mobility. The present article examines job moves between East, North, and South Germany and the related income perspectives. The data basis is a Linked Employer-Employee data set which has been supplemented with regional structural indicators. The results of the analyses show: Younger and highly qualified employees change more frequently and are more likely to increase their income when they change companies. Incentives for East-West mobility persist, because for trajectories from East to North or South Germany, adjusted for regional price level, a comparatively higher probability of income success can be determined. Transitions to East Germany can be associated with a loss of income but also a gain of income.

$$
\text { JEL-Klassifizierung: J62 }
$$




\section{Einleitung}

In Deutschland bestehen erhebliche wirtschaftliche Unterscheide zwischen den Großregionen Ost-, Nord- und Süddeutschland (siehe auch Abschnitt 5.1). Dabei haben sich die Differenzen zwischen West- und Ostdeutschland - ungeachtet der anfänglich erheblichen Angleichungsprozesse nach der Wiedervereinigung (Struck 2017) und der insgesamt positiven Entwicklung auf dem Arbeitsmarkt (Eichhorst et al. 2017) - in den letzten Jahren sogar noch vergrößert (Albrech et al. 2016). Für Menschen, die in strukturschwachen Regionen leben und arbeiten, bestehen Anreize, ihren Arbeits- und Wohnort in wirtschaftlich leistungsstarke und vermeintlich chancenreichere Regionen zu verlagern. Werden dabei große Distanzen zwischen den Landesteilen überwunden, dann geht dies vielfach mit erheblichen ökonomischen und soziale Kosten sowie kulturellen Veränderungen einher. Diesen stehen wiederum Erwartungen von Einkommenszuwächsen entgegen.

Es ist bekannt, dass gerade jüngere und gebildete Personengruppen vergleichsweise häufiger am Arbeitsmarkt mobil sind (Granato et al. 2009; Haas 2000; Ruppenthal, Windzio 2004a). Ebenso haben sich in den letzten 30 Jahren viele Menschen beruflich von Osten in den Westen bewegt. Allerdings existieren kaum empirische Untersuchungen, die Erfolgsbedingungen und finanzielle Erfolgschancen räumlicher Mobilitätsprozesse in den Blick nehmen. Vor diesem Hintergrund untersucht der Beitrag, in welchem Umfang ein Arbeitsplatzwechsel zwischen den Großregionen Ost-, Nord- und Süddeutschland finanziell erfolgreich ist und welche Faktoren auf einen Einkommenszuwachs wirken. Dies erfolgt auf Basis eines Linked EmployerEmployee Datensatzes, der durch regionale Strukturindikatoren ergänzt wurde. In die multivariaten Analysen gehen die Individualmerkmale der Beschäftigten, aber auch Charakteristika der Zielbetriebe und wirtschaftsstrukturelle Merkmale der Zielregionen ein.

\section{Stand der Forschung}

Migrationsströme innerhalb Deutschlands sind vergleichsweise gut dokumentiert (Sander 2014; Schlömer 2004; Statistisches Bundesamt 2015). Ein Großteil der Binnenwanderung in Deutschland erfolgt lokal innerhalb von Kreisen oder über benachbarte Kreisgrenzen hinweg. Zudem wurden individuelle Wanderungsmuster identifiziert (Buch 2006; Ganesch 2018; Hunt 2006; Windzio 2004a). Die hier im Vordergrund stehende großräumige berufliche Mobilität ist entsprechend seltener und war dabei in den letzten Jahren zudem stark von der deutsch-deutschen Vereinigung beeinflusst. Nach dem Fall der Mauer kam es zu einer massiven Abwanderung aus Ostdeutschland. Die anfänglich sehr hohen negativen Wanderungssalden verringerten sich bis Mitte der 1990er Jahre, wobei immer noch viele Menschen aus dem Osten in den Westen zogen, zugleich aber auch sogenannte „Aufbauhelfer" aus dem Westen in Ostdeutschland arbeiteten. Nach einem erneuten Anstieg des negativen Wanderungssaldos von 1997 bis Anfang der 2000er Jahre gingen die Wander- 
ungsverluste besonders seit dem Jahr 2008 wieder zurück (Windzio/Huinink 2010, S. 10 f.; Windzio 2013, S. 669).

Unabhängig davon, ob etwa von Ost nach West oder Nord nach Süd gewandert wird, ist bekannt, dass gerade jüngere und gebildete Personengruppen vergleichsweise häufiger am Arbeitsmarkt mobil sind (Ganesch et al. 2017; Granato et al. 2009; Haas 2000; Ruppenthal 2010; Windzio 2004a). Zudem korrespondiert die regionale Mobilitätsneigung positiv mit der Höhe des individuellen Tagesentgelts (Reichelt/Abraham 2015; Windzio 2004b).

Darüber hinaus stellen einige Studien die Relevanz regionaler Kontexte bei Mobilitätsentscheidungen heraus. Bezüglich der regionalen Qualifikationsstruktur zeigt sich, dass insbesondere eine berufsfachlich qualifizierte bzw. (hoch)qualifizierte Beschäftigtenstruktur das Beschäftigungswachstum einer Region positiv beeinflusst (Blien/Wolf 2002; Farhauer/Granato 2006; Schlitte et al. 2010; Shapiro 2006; Südekum 2008). Diese Befunde legen nahe, dass ein hoher Anteil an qualifizierten Beschäftigten ein regionaler Prosperitätsfaktor zu sein scheint und davon eine Sogwirkung auf Arbeitskräfte ausgeht (Buch et al. 2014; Arntz et al. 2014). Die skizzierten Analyseergebnisse lassen allerdings offen, ob und inwieweit die aufgezeigten Einflussfaktoren die Wahrscheinlichkeit, bei räumlicher Mobilität tatsächlich selbst Einkommenserfolge oder -verluste erzielen zu können, erhöhen.

Aufstiegsfördernde Effekte regionaler Mobilität in den ersten fünf Jahren der Erwerbskarriere können Reichelt/Abraham (2015) feststellen. Zu Beginn der Erwerbskarriere können Beschäftigte eher von der Verlagerung des Arbeitsortes in einen anderen Regionstypen profitieren als im späteren Erwerbsverlauf (Lehmer/Ludsteck 2011, S. 558). Bei großräumiger Arbeitsortmobilität ${ }^{1}$ (Entfernung zwischen den Arbeitsplätzen mit Fahrtzeiten von mindestens einer Stunde) fällt der Einkommenszuwachs jüngerer Beschäftigter noch höher aus (ebd., S. 565). Hacket (2009) zeigt für die Gruppe der Berufsanfänger, dass allein Hochqualifizierte bei Betriebswechseln von West- nach Ostdeutschland Einkommenserfolge haben, wohingegen Beschäftigte ohne beruflichen Abschluss hohe Verlustrisiken haben. Bei einem Verlassen des Ausgangsarbeitsorts in Ostdeutschland in Richtung Westdeutschland sind die Chancen, unabhängig vom Bildungsniveau Einkommensgewinne zu erzielen, höher und die Verlustrisiken geringer als bei regionalen Arbeitsplatzwechseln innerhalb Ostdeutschlands (ebd.). Zu berücksichtigten ist jedoch, dass die zum Teil erheblichen regionalen Preisunterschiede hier nicht berücksichtigt wurden.

\footnotetext{
${ }^{1}$ Hinweis: räumliche Mobilität wird als Veränderung der Besiedlungsdichte im Zielraum verglichen mit dem Ausgangsraum definiert.
} 
Kratz/Brüderl (2013) untersuchen mit regionaler Mobilität einhergehende Einkommenseffekte für in Westdeutschland lebende Männer ${ }^{2}$ auf Basis der Daten des SOEP (1984-2010) und stellen fest, dass regionale Mobilitätsprozesse einkommensförderlich sind. Maier/Sprietsma (2016) weisen auf Basis der Daten des NEPS für Hochschulabsolventen positive Einkommenseffekte im Erwerbsverlauf nach, sofern diese zu Beginn ihrer Erwerbskarriere regional mobil sind. Für regional mobile Frauen werden signifikant schlechtere Aufstiegschancen ${ }^{3}$ ermittelt (Ganesch et al. 2017; Hillmert 2006, S. 3398).

Betrachtet man bundesländerübergreifende Wanderungsprozesse, so stellen die Metropolen Hamburg, München und Berlin beliebte Zielräume dar (Sander 2014). Positive Einkommensentwicklungen weisen Glaeser/Maré (2001) nach, wenn Beschäftigte in Agglomerationsräume wechseln. In Metropolregionen, welchen aufgrund der hohen Konzentration des Arbeitsangebots und der Arbeitsnachfrage eine verbesserte Zuordnung von Arbeitskräften und Arbeitsplätzen zugeschrieben wird (Glaeser/Maré 2001; Wheeler 2006), liegen die Löhne räumlich mobiler Beschäftigter allerdings unmittelbar nach dem Wechsel unter dem Lohnniveau der in der jeweiligen Zielregion bereits erwerbstätigen Arbeitskräfte (ebd.). Lehmer/Möller (2008) beobachten positive Einkommensverläufe nach regionaler Mobilität unabhängig vom regionalen Zielkontext. Bei Lehmer/Ludsteck (2011, S. 559) finden sich für ländliche Zielregionen hingegen negative Einkommenseffekte.

Werden betriebliche Charakteristika in die Analyse einbezogen, so zeigt sich, dass höhere Lohnzuwächse in urbanen Räumen größtenteils durch eine Beschäftigung in Großbetrieben mit einer ausgesprochen guten Qualifikationsausstattung erklärt werden können (Kelle 2016). Unter der Kontrolle betrieblicher Kontextmerkmale kann nur ein sehr geringer Teil der beobachteten positiven Einkommensentwicklungen in Agglomerationsräumen dem unterstellten effizienteren Zuordnungsprozess von Arbeitskräften zu Arbeitsplätzen in urbanen Räumen zugeschrieben werden (ebd.).

Insgesamt deutet sich an, räumliche Mobilität lohnt sich finanziell für viele Beschäftigte. Die Wahrscheinlichkeit, dass sich durch räumliche Mobilität die Einkommenshöhe verbessert, ist höher, als die Wahrscheinlichkeit, dass sie sich verschlechtert. Dies ist zu erwarten: Eine Einkommensverbesserung wird vielfach beim Arbeitgeberwechsel vereinbart bzw. angestrebt. Die Frage ist allerdings, welche Faktoren sind dafür verantwortlich, dass ein substanzieller (preisbereinigter) Einkommenszuwachs tatsächlich eintritt. Bisherige Analysen haben versucht einige wichtige zu berücksichtigende Einflussgrößen auf die

\footnotetext{
${ }^{2}$ Betrachtet werden lediglich Männer, die zum ersten Erhebungszeitpunkt in Westdeutschland lebten. Ostdeutsche sind nicht Teil der Untersuchungsgruppe, da die Autoren argumentieren, dass

Wanderungsbewegungen im Zuge der deutschen Einigung eigenen spezifischeren Mustern folgen.

${ }^{3}$ Hillmert (2006) operationalisiert berufliche Aufstiegschancen anhand von Niveauveränderung der beruflichen

Stellung, die nicht unbedingt auch finanzielle Folgen haben.
} 
Einkommensentwicklung im Zuge räumlicher Mobilitätsprozesse zu identifizieren. Allerdings sind dabei jeweils viele der individuellen, betrieblichen und regionalen Merkmale unkontrolliert geblieben, da sie nicht gemeinsam in einer Untersuchung analysiert wurden. Zudem werden lokale Preisunterschiede bei der Operationalisierung von Einkommensveränderungen im Zuge räumlicher Mobilität meist nicht berücksichtigt. Diese Lücken versucht der vorliegende Beitrag zu schließen. Dazu wird der Einfluss von Individualmerkmalen und regionaler Mobilität unter der Kontrolle betrieblicher und regionaler Charakteristika des Zielbetriebskontextes auf die Wahrscheinlichkeit, preisniveaubereinigt Einkommenserfolge erzielen zu können, untersucht.

\section{Theoretischer Rahmen und Hypothesen}

Die individuelle Neigung zu räumlicher Mobilität bei Arbeitsplatzwechseln wird zumeist aus der Perspektive nutzentheoretischer Ansätze erklärt. Gemäß dem place-utility-Ansatz (Wolpert 1965, S. 161 ff.) resultieren Entscheidungen zu überregionaler Mobilität aus dem Vergleich der Gegebenheiten und Nutzenwerte (place utility) am Ziel- und Herkunftsort. Dabei können sowohl die mit räumlichen Mobilitätsentscheidungen einhergehenden beruflichen, wie auch private Konsequenzen (darunter etwa familiale und soziale Bindungen) von Bedeutung sein. Diese Auswirkungen können bei großen Distanzen und Mobilität zwischen wirtschafts- und sozialstrukturellen sowie kulturell unterschiedlichen Landesteilen wie hier in Ost-, Nord- und Süddeutschland besonders tiefgreifend sein. Räumliche Mobilität wird dann zur Handlungsoption, wenn das Anspruchsniveau die Nutzenschwelle des bisherigen Arbeitsorts übersteigt und/oder die Nutzenschwelle eines alternativen Arbeitsorts die des bisherigen Arbeitsortes überschreitet. Auch aus der Perspektive der Suchtheorie kommt es zu Arbeitsortmobilität, wenn die Such- und Mobilitätskosten durch Einkommenszuwächse in der Zielbeschäftigung kompensiert werden können (McKenna 1985; Kalter 1994, S. 471). Vor diesem Hintergrund sollte dann allgemein gelten:

H1: Im Zuge regionaler Arbeitsplatzmobilität zwischen Großregionen werden mit höherer Wahrscheinlichkeit Einkommenszuwächse realisiert als bei Wechseln innerhalb der von Großregion.

Wie aus der Betrachtung regionaler Strukturindikatoren hervorgeht, sind die lokalen Rahmenbedingungen nicht in allen Zielregionen gleich günstig. Zwischen Nord-, Süd- und Ostdeutschland bestehen hinsichtlich der wirtschaftlichen Leistungsfähigkeit, der Arbeitslosigkeit oder der Siedlungsdichte sehr heterogene Ausgangslagen (Albrech et al. 2016; Statistisches Bundesamt 2016, S. 350 ff.; siehe im Folgenden Kapitel 5.1). Gleichwohl besten auch innerhalb der Großregionen Unterschiede insbesondere zwischen städtischen und ländlichen Regionen. Es ist sinnvoll, diese Strukturen bei der Suche nach Erfolgsursachen 
von Mobilität auch zwischen wirtschaftlich stark unterschiedlichen Landesteilen mit zu berücksichtigen.

Regionalökonomischen und raumstrukturellen Ansätzen folgend können regionale Strukturmerkmale zu Einkommenserfolgen beitragen. Gemäß dem Zentrum-Peripherie-Modell (Krugman 1991) nutzen Betriebe und Arbeitskräfte in urbanen Räumen Agglomerationsvorteile aus. Ein Betrieb versucht im Falle hoher Skalenerträge, die Produktion auf einen einzigen Standort zu beschränken und bedient von dort aus den Markt. Um Transportkosten zu vermeiden, siedeln sich Betriebe in Regionen mit großer Nachfrage, d. h. einer hohen Bevölkerungsdichte an. Dadurch wiederum werden Arbeitskräfte und weitere Betriebe attrahiert. Letztere realisieren Agglomerationsvorteile infolge eines großen Absatzmarktes oder eines großen Arbeitskräftepools in einer solchen Region (ebd.). Regionalökonomische Forschungen können eine stärkere Arbeitskräftenachfrage und somit vergleichsweise gute Beschäftigungschancen in dicht besiedelten Regionen bestätigen (Blien et al. 2006; Farhauer/Granato 2006). Das in zentralen Räumen breitere Arbeitsplatzangebot erhöht zum einen die Beschäftigungschancen der wechselaffinen Person selbst und kann - falls in Partnerschaften Haushaltumzüge vollzogen werden - auch der/dem Lebenspartner(-in) zugutekommen.

Gemäß dem dualen Raumkonzept nach Fassmann/Meusburger (1997) bildet sich dann ausgehend von einer stabilen Güternachfrage und der Ansiedlung leistungsfähiger Unternehmen ein primäres Arbeitsmarktsegment in zentralen Räumen aus, das durch stabile Arbeitsplätze, gute Bezahlung, Aufstiegschancen sowie vorwiegend gut qualifizierte Arbeitskräfte gekennzeichnet ist. In peripheren Räumen entwickelt sich hingegen aufgrund der instabilen Nachfrage und weniger leistungsstarker Unternehmen ein sekundäres Arbeitsmarktsegment mit instabilen und gering bezahlten Arbeitsverhältnissen, geringen Qualifikationsanforderungen und wenigen Aufstiegschancen. Demzufolge dürften die regionalen arbeitsmarktstrukturellen Bedingungen in wirtschaftlich starken, zentralen Lagen die Einkommensentwicklung positiv beeinflussen.

Aus der Perspektive der Such- und Matchingtheorie (Wheeler 2001, 2006) ermöglichen dichtbesiedelte Räume aufgrund der hohen Konzentration des Arbeitsangebots und der Arbeitsnachfrage eine verbesserte Zuordnung von Arbeitskräften und Arbeitsplätzen hinsichtlich der Qualifikationsanforderungen (Glaeser/Maré 2001). Mit der Größe des lokalen Arbeitsmarktes nehmen das Arbeitsangebot und die Arbeitsnachfrage zu. Somit erhöhen sich zunächst die potentiellen Übereinstimmungsmöglichkeiten zwischen Arbeitsplatzsuchenden und vakanten Arbeitsstellen als auch die Anzahl der erfolgreich besetzten Arbeitsplätze. Aufgrund der geringeren Suchkosten und der höheren Chancen auf ein passendes Matching 
ergeben sich in zentralen Lagen Produktivitätsvorteile, die die Wahrscheinlichkeit von Einkommenszuwächsen erhöhen dürften (Jovanovic 1979).

H2: Großregionenwechsel in Richtung agglomerierter Zielräume sowie in Räume mit positiven wirtschaftsstrukturellen Kennziffern erhöhen (a) die Wahrscheinlichkeit substanzieller Einkommenszuwächse und (b) verringern das Risiko von Einkommensverlusten.

Darüber hinaus hängt das räumliche Mobilitätsverhalten humankapitaltheoretischen Überlegungen zufolge stark von den individuellen Bildungsressourcen ab. Qualifikationsspezifische Unterschiede hinsichtlich der räumlichen Mobilitätsneigung können der Suchtheorie zufolge dadurch erklärt werden, dass die oftmals sehr spezifischen Qualifikationsanforderungen im Tätigkeitsbereich der Hochqualifizierten teilweise nur in spezialisierten überregionalen Arbeitsmärkten nachgefragt werden (Damelang 2007, S. 7). Beschäftigte mit höheren Qualifikationen und spezifischen Kompetenzprofilen, die ihren Arbeitsplatz wechseln möchten, haben auf dem überregionalen Arbeitsmarkt vergleichsweise bessere Chancen einen qualifikationsadäquaten und gut bezahlten Arbeitsplatz zu finden (Mertens/Haas 2006). Arbeitsortmobilität kann als Zusatzinvestition zur Verbesserung der Verwertbarkeit von Humankapital begriffen werden und Einkommens- und Erwerbschancen verbessern (ebd; Sjaastad 1962).

H3: Hochqualifizierte, die zwischen Großregionen betriebsmobil sind, können mit einer höheren Wahrscheinlichkeit Einkommenszuwächse realisieren.

Die mit räumlichen Mobilitätsprozessen einhergehenden Kosten und der Nutzen sind darüber hinaus eng mit dem Lebensalter bzw. der individuellen Lebensphase verbunden. Aus der Perspektive der Lebensverlaufsforschung treten im frühen Erwachsenenalter verstärkt Wendepunkte im Lebenslauf (Erwerbseintritt, das Zusammenziehen mit einer Partnerin oder einem Partner, etc.) auf (Huinink/Kley 2008). In diesen Orientierungs- und Entscheidungsphasen weisen Beschäftigte in der Regel entsprechend dem Job Shopping-Ansatz (Johnson 1978) eine hohe Arbeitsplatzmobilität auf, um verschiedene Beschäftigungsoptionen auszutesten (Haas 2000, S. 6 allg. auch Boyle et al. 1998). Mit zunehmendem Alter sind berufliche und private Wahl- und Entscheidungsprozesse, wie beispielsweise hinsichtlich der Arbeitgeber-, Wohnort- sowie Partnerwahl, aber auch der Heirat und Kinderplanung, weitgehend verwirklicht und demnach die soziale Einbettung in einen Betrieb und eine Region gefestigter. Aus diesem Grund ist davon auszugehen, dass die privaten Kosten, die aufgrund der räumlichen Trennung vom Haushaltskontext und vom sozialen Umfeld oder durch einen Haushaltsumzug mit erwerbsbedingter räumlicher Mobilität einhergehen, im Verlauf des Lebens tendenziell steigen und der relative Nutzen überbetrieblicher Mobilität sinkt. Wechseln ältere Beschäftigte dennoch ihren Arbeitsplatz 
zwischen Großregionen, dann sind substanzielle Einkommenszuwächse für die Gruppe der älteren regional mobilen Beschäftigten unter Kontrolle der Betriebszugehörigkeitsdauer im vorherigen Beschäftigungsverhältnis zu erwarten.

H4: Ältere Beschäftigte, die zwischen den Großregionen betriebsmobil sind, erzielen mit höherer Wahrscheinlichkeit substanzielle Einkommenszuwächse.

Die Lohnspannen sind bei Frauen und Männern unterschiedlich. Damit zusammenhängend sind Frauen deutlich häufiger in Sozial- und Gesundheitsberufen, im kaufmännischen Bereich sowie im öffentlichen Dienst beschäftigt als Männer (Hausmann/Kleinert 2014; Hinz/Gartner 2005; Statistisches Bundesamt 2017, S. 32). Diese Berufskorridore stellen vergleichsweise gute direkte überbetriebliche Mobilitätschancen bereit. Hinsichtlich der Entlohnung sind etwas geringere Durchschnittslöhne aber auch geringere Spielräume für größere Einkommenszuwächse als in den von Männern dominierten technischen und IT-Berufen festzustellen (ebd.; Wirtschafts- und Sozialwissenschaftliches Institut 2018). Darüber hinaus ist davon auszugehen, dass Entscheidungen, den Arbeitsplatz überregional zu wechseln, die in der Regel tiefgreifende Veränderungen im privaten Bereich mit sich bringen, in relativ gut abgesicherten Ausgangslagen der Berufsgruppen nicht getroffen werden, sofern damit Einkommensverschlechterungen verbunden sind. Angesichts dieser strukturellen Merkmale sollte gelten:

H5: Für Frauen, die ihren Arbeitsplatz zwischen Großregionen wechseln, ist die Wahrscheinlichkeit von Einkommenszuwächse oder -einbußen geringer als bei Männern.

In den Analysen sind weitere, strukturelle Einflussfaktoren zu kontrollieren, so etwa der Konjunkturverlauf (Hinz/Abraham 2008; Struck 2006). Unter der Annahme, dass im konjunkturellen Aufschwung bei steigendem Arbeitsangebot zahlreiche, hinsichtlich der Entlohnung attraktive Arbeitsplätze entstehen, wird sowohl die zwischenbetriebliche wie auch die interregionale Mobilität steigen. Hingegen werden in einer Rezession nur wenige attraktive Arbeitsstellen geschaffen bzw. Arbeitsplätze abgebaut. Zudem sind kaum Spielräume für Lohnerhöhungen vorhanden. Damit sinkt der Anreiz für freiwillige räumliche Mobilität, während unfreiwillige Freisetzungen zunehmen (Struck et al. 2007; Giesecke/Heisig 2010). Anzunehmen ist daher, dass die erwerbsbedingte regionale Mobilität prozyklisch verläuft. Im konjunkturellen Aufschwung kommt es - anders als in Phasen des Abschwungs - verstärkt zu überregionalen Beschäftigungsangeboten mit attraktiven Einkommensanreizen, die regionale Mobilitätsprozesse nach sich ziehen. Aufgrund der vorgelagerten Selektion für Mobilitätsentscheidungen erwarten wir geringe und allein in Jahren eines Aufschwunges moderate Einkommenszuwächse im Zuge räumlicher Mobilitätsprozesse. 
Im Zuge des „new structuralism“ (Baron/Bielby 1980) sowie in Ansätzen zu betrieblichen Beschäftigungssystemen (Hendry 2003; Lepak et al. 2006; Struck 2006) wird die Bedeutung von Betriebsmerkmalen für Erwerbschancen und -risiken betont. Empirische Befunde können die Relevanz des Zielbetriebskontextes belegen (Kelle 2016). Der betriebliche Kontext kann auch unabhängig von der lokalen wirtschaftlichen Lage direkt Einfluss auf die Aufstiegschancen und Abstiegsrisiken der Beschäftigten nehmen. Aus diesem Grund werden bei der Analyse des räumlichen Arbeitsmarktverhaltens neben den wirtschafts- und siedlungsstrukturellen Gegebenheiten ebenso Betriebsmerkmale, wie das Lohnniveau und der Wirtschaftszweig, kontrolliert.

\section{Datengrundlage, Operationalisierung und Methode}

\subsection{Datengrundlage}

Die Datengrundlage für die nachfolgenden Analysen bildet der integrierte Betriebs- und Personendatensatz (LIAB) des Instituts für Arbeitsmarkt- und Berufsforschung (IAB). Dieser verbindet die administrativen Arbeitnehmerdaten der Historikdatei der Beschäftigtenstatistik der Bundesagentur für Arbeit, die sehr valide Angaben zu Beschäftigungszeiten und den Löhnen enthalten, mit den Betriebsinformationen aus den jährlichen Befragungen des IABBetriebspanels (Jacobebbinghaus 2008). Durch die Integration der Informationen aus der Leistungsempfängerdatei in die Beschäftigtenstatistik lassen sich die Erwerbsbiografien der sozialversicherungspflichtig Beschäftigten (inklusive Phasen der Nicht-Erwerbstätigkeit) nachzeichnen. Der verwendete LIAB-Längsschnittdatensatz (Version: LIAB LM 9310) beinhaltet die Erwerbsverläufe aller Personen, die mindestens einen Tag zwischen dem 01.01.2000 und 31.12.2009 in einem Betrieb des Betriebspanels sozialversicherungspflichtig beschäftigt waren. Diese Datenbasis wurde um ausgewählte regionale Strukturindikatoren auf der Ebene der Raumordnungsregionen im jeweiligen Wechseljahr ergänzt, welche der INKARDatensatz des Bundesinstituts für Bau-, Stadt-, und Raumforschung (BBSR) bereitstellt. Die Analysen zu Einkommensveränderungen beziehen sich auf betriebsmobile Vollzeitbeschäftigte im Alter von 18 bis 65 Jahren, für welche betriebliche und regionale Merkmale des Zielraumes berichtet werden. ${ }^{4}$ Das Analysezeitfenster erstreckt sich dabei von 2000 bis Ende 2010. ${ }^{5}$

\footnotetext{
${ }^{4}$ Episoden mit einem Einkommen, das jenseits der Beitragsbemessungsgrenze der gesetzlichen Rentenversicherung liegt und daher zensiert ist, sind nicht im Analysesample enthalten.

${ }^{5}$ Deskriptionen der Erwerbsspells im Analysesample sind in Anhang 1 dokumentiert.
} 


\subsection{Operationalisierung und Methodik}

Erwerbsbedingte regionale Mobilität wird - unabhängig davon, ob es sich hierbei um punktuelle oder zirkuläre ${ }^{6}$ Mobilität handelt - als räumliche Veränderung des ursprünglichen Arbeitsortes über die Grenzen von Großregionen hinweg operationalisiert. Als Großregionen werden Nord, Süd und Ostdeutschland klassifiziert, die entsprechend ihrer geografischen und wirtschaftsstrukturellen Lage abgegrenzt werden (siehe Abbildung 1 im Anhang). Darüber hinaus wurden innerhalb der Großregionen lokale Strukturindikatoren auf der Ebene von 96 Raumordnungsregionen (BBSR 2016) an den LIAB-Datensatz gespielt. Damit ist die räumliche Kategorisierung in der Lage grundlegende ost-/westspezifische Unterschiede hinsichtlich der regionalen Arbeitsmarktsituation und des Lohnniveaus abzubilden und zudem das messbare Nord-/ Südgefälle aufzugreifen.

Direkte Betriebswechsel sind als Übergänge aus einer betrieblichen Vollzeitbeschäftigung in eine erneute Vollzeitbeschäftigung definiert, um individuelle Einkommensveränderungen vor dem Hintergrund eines vergleichbaren Arbeitszeitumfangs analysieren zu können. Dies ist nötig, da in den administrativen Daten die Arbeitszeiten nicht erfasst sind. Kurze Phasen der Nicht-Erwerbstätigkeit von maximal 90 Tagen werden ausgeblendet, da davon auszugehen ist, dass die Beschäftigten den zukünftigen Arbeitgeber zu dem Zeitpunkt, zu dem die vorangehende betriebliche Beschäftigung endet, meist bereits kennen. Als Übergänge in Nicht-Erwerbstätigkeit werden Folgeepisoden der Arbeitslosigkeit oder Sozialversicherungslücken mit einer Dauer von mehr als 90 Tagen angesehen.

Die abhängige Variable unserer Analyse stellen die unmittelbaren Einkommensveränderungen im Zuge räumlicher Arbeitsortverlagerung dar. Unterschieden wird dabei zwischen Einkommensaufstiegen, lateraler Mobilität und Einkommensabstiegen. Als Aufstiege werden Lohnzuwächse von mehr als $10 \%$ bezeichnet, während bei einem Rückgang des Lohnes von mehr als $5 \%$ von einem Abstieg ausgegangen wird. ${ }^{7}$ Einkommensstabilität - laterale Mobilität - liegt dann vor, wenn sich das Einkommen lediglich bis maximal $10 \%$ erhöht bzw. um bis zu $5 \%$ sinkt. Um nicht absolute, sondern reale Einkommenssituationen vergleichen zu können, werden die Einkommen auf Basis von Daten

\footnotetext{
${ }^{6}$ Unter punktueller Mobilität ist eine einmalige (erwerbsbedingte) Wohnortmobilität zu verstehen. Zirkuläre Mobilität meint im Gegensatz dazu das regelmäßige Pendeln zum Arbeitsplatz.

7 Der Definition von Auf- und Abstiegen liegen asymmetrische Grenzen zugrunde, da anzunehmen ist, dass Verlustaversion besteht (Novemsky/Kahneman 2005) und Einkommensverluste bereits in kleinerem Ausmaß als Situationsverschlechterung empfunden werden. Schätzmodelle, die symmetrische Grenzen der abhängigen Variable „Einkommensveränderungen“ zugrunde legen, führen zu nahezu unveränderten Prädiktorwerten bzw. Relative Risk Ratios.
} 
des BBSR (Kawka 2009) um das lokale Preisniveau der Herkunfts- und Zielarbeitsorte auf der Ebene der Kreise bzw. Kreisregionen bereinigt. ${ }^{8}$

Die Analysemodelle beziehen neben Individualmerkmalen (Geschlecht, Alter, berufliches Qualifikationsniveau sowie Nationalität, Höhe des bisherigen Tagesentgelts), die räumliche Lagebeziehung des Ausgangsarbeitsplatzes (die jeweilige Großregion sowie die Grenznähe des Arbeitsortkreises zu der bzw. den anderen Großregion(en)) die Arbeitsortmobilität zwischen Großregionen und ihre Richtungen sowie betriebliche und regionale Charakteristika mit ein. Aufgrund der insbesondere zu Beginn von betrieblichen Beschäftigungen hohen Ausstiegsraten (Grotheer/Struck 2003), die sich nach circa zwei Jahren sukzessive stabilisieren, wird die Betriebszugehörigkeitsdauer beim bisherigen Arbeitgeber kategorial anhand folgender Betriebszugehörigkeitskohorten erfasst: Bis zu zwei Jahren, zwei bis sieben Jahre sowie mehr als sieben Jahre in einem Betrieb beschäftigt. Auf der Betriebsebene wird das Lohnniveau (als Medianeinkommen der Vollzeitbeschäftigten) sowie die Branche der Zielbetriebsstätte kontrolliert. Die wirtschaftsregionalen Charakteristika der Zielräume werden auf der Ebene von Raumordnungsregionen gemessen. Dieses raumstrukturelle Analysekonzept umfasst 96 Raumordnungsregionen, welches auf der räumlichen Einheit der Kreise basiert und sowohl wirtschafts- und arbeitsmarktstrukturelle Gegebenheiten als auch Pendelbeziehungen zwischen dem Umland und den Oberzentren berücksichtigt (BBSR 2016). Somit bilden Raumordnungsregionen regionale Arbeitsmarktbedingungen adäquat ab (Rendtel/Schwarze 1996). Als regionale Strukturmerkmale werden die Siedlungsstruktur (in Form des Regionstyps), die Arbeitslosigkeitsquote, die wirtschaftliche Leistungsfähigkeit (anhand des regionalen Bruttoinlandsproduktes pro Einwohner) und das durchschnittliche Arbeitnehmerentgelt sowie die regionale Qualifikationsstruktur als Anteil der hochqualifizierten sozialversicherungspflichtig Beschäftigten (gemessen an je 1000 Einwohnern im erwerbsfähigen Alter) berücksichtigt. Weiterhin werden generelle Einflüsse der konjunkturellen Situation auf Basis von Jahresdummies kontrolliert.

Die Einkommensveränderungen werden auf Basis von multinomialen logistischen Modellen geschätzt. Primäres Ziel ist es, räumliches Arbeitsmarktverhalten und damit einhergehende Einkommensveränderungen unter Einbeziehung der relevanten Faktoren auf allen Analyseebenen zu untersuchen. Die Messung der Einflussstärke der einzelnen Ebenen ist sekundär. Um die hierarchische Datenstruktur abzubilden, werden clusterrobuste Standardfehler auf der Ebene der 96 Raumordnungsregionen berechnet. Die Anzahl der

\footnotetext{
${ }^{8}$ Arbeitsplatzwechsel in Raumordnungsregionen mit einem höheren regionalen Preisniveau müssen nicht zwangsläufig mit einem Umzug in diese Region und einer dadurch bedingten Reduktion des Realeinkommens verbunden sein. Jedoch fallen in diesem Fall Pendelkosten an, die das Realeinkommen schmälern, selbst wenn der ursprüngliche Wohnort in einer Region mit einem geringeren regionalen Preisniveau als dem des Zielarbeitsortes beibehalten wird. Berichtet und interpretiert werden die preisniveaubereinigten vertikalen Mobilitätsprozesse.
} 
Cluster ist ausreichend groß, um die Standardfehler unverzerrt ermitteln zu können (Kézdi 2004; Angrist/Pischke 2009).

\section{Ergebnisse}

Zunächst werden die raumstrukturellen Disparitäten skizziert und das Analysesample charakterisiert. Daran schließt sich die Ergebnispräsentation an.

\subsection{Deskriptionen}

Eigene Berechnung zu raumstrukturellen Indikatoren auf Basis der INKAR-Daten verdeutlichen zunächst, dass zwischen den aus den Daten extrahierten drei Großregionen erhebliche Niveauunterschiede bezüglich der Arbeitsmarktlage, der wirtschaftlichen Leistungsfähigkeit und des lokalen Lohnniveaus bestehen (siehe beispielhaft die Darstellung der Arbeitslosigkeitsquoten auf der Ebene von Raumordnungsregionen im Jahr 2010 in Abbildung 1 im Anhang). Während sich der Großraum Süddeutschland positiv vom Bundesdurchschnitt abhebt, weist Ostdeutschland hinsichtlich aller Indikatoren deutlich ungünstigere Werte auf. Süd- und Ostdeutschland bestehen aus vergleichsweise homogenen, strukturähnlichen Raumordnungsregionen. Norddeutschland ist in sich etwas heterogener, $d$. h. von strukturstarken als auch strukturschwachen Raumordnungsregionen gekennzeichnet. Siedlungsstrukturell bestehen ebenfalls Differenzen. Zwölf der insgesamt 24 städtischen Regionen ${ }^{9}$ befinden sich im Norden, neun im Süden und nur drei im Osten Deutschlands. Ähnlich stellt sich die Verteilung der Raumordnungsregionen mit Verstädterungstendenz dar (Nord: 16; Süd: 15; Ost: 4). In Ostdeutschland dominieren ländlich geprägte Raumordnungsregionen (15). Diese peripheren Strukturen finden sich im Norden vergleichsweise selten (6), sind aber im Süden neben den urbanen Regionen ebenfalls anteilig bedeutsam (16).

Zwischen diesen Regionen werden Arbeitsplätze gewechselt. Von knapp 185.000 im Analysesample beobachteten Betriebswechseln erfolgen 8,6\% (15.974) zwischen Großregionen. Hierbei sind dann, wie auch insgesamt in der Literatur beschrieben jüngere Beschäftigte, Männer und akademisch Gebildete vergleichsweise häufiger mobil (siehe Anhang 2). Deskriptiv zeigt sich auch, mit Betriebswechsel, die über die Großregion hinaus erfolgen, gehen zu einem Großteil (51\%) deutliche Einkommenszuwächsen (>10\%) einher. In

\footnotetext{
${ }^{9}$ Diese Kategorisierung basiert auf den Angaben, die das Bundesinstitut für Bau-, Stadt-, und Raumforschung (BBSR) in den INKAR-Daten ausgibt.
} 
$28,1 \%$ sind Einkommensverluste $(>5 \%)$ und in $20,9 \%$ keine größeren

Einkommensveränderungen zu beobachten.

\subsection{Multivariate Befunde}

Um zu überprüfen, welche Faktoren für den Einkommenserfolg regionaler Mobilitätsprozesse bedeutsam sind, werden im Folgenden die Ergebnisse multivariater Analysen vorgestellt. Die nachfolgenden multinomialen logistischen Schätzmodelle weisen Relative-Risk-Ratios ${ }^{10}$ aus. Das Baseoutcome der abhängigen Variable „relative Einkommensveränderungen“ stellt hierbei die Ausprägung „keine substantiellen Einkommensveränderungen - laterale Mobilität“ dar.

\section{Etwa hier Tabelle 1: Multinomiale Schätzmodelle zu vertikalen Mobilitätsprozessen betriebsmobiler Beschäftigter}

Zunächst wird der Einfluss von Arbeitsortmobilität zwischen Großregionen auf substantielle Einkommensveränderungen betrachtet (Tabelle 1). Diese wurden um lokale Preisunterschiede bereinigt. Unter Kontrolle der Ausgangsregion, der Entfernung zu einer Großregion und individueller Merkmale sowie der betrieblichen und regionalen Charakteristika des jeweiligen Zielbetriebskontextes ist festzustellen, dass Arbeitsortmobilität zwischen Großregionen die Wahrscheinlichkeit substanzielle Einkommenserfolge zu erzielen, im Vergleich zu lokalen Betriebswechseln erwartungsgemäß erhöht (Modell 1; Hypothese 1). Doch welches sind die Erfolg fördernden Faktoren?

In Modell 2 werden die Wanderungsrichtungen zwischen den Großregionen Nord-, Süd- und Ostdeutschland einbezogen. ${ }^{11}$ Hierbei lässt sich für Wechsel aus Ostdeutschland in Richtung Nord- und in Richtung Süddeutschland preisbereinigt eine höhere Einkommenserfolgswahrscheinlichkeit nachweisen als für Betriebsmobile innerhalb Ostdeutschlands. Somit bestehen die von Hacket (2009) für die Folgejahre der deutschen Wiedervereinigung festgestellten Einkommenschancen bei Ost-West-Mobilität bis 20 Jahre nach der Wende, und auch bei Berücksichtigung regionale Preisunterschiede fort. Eine Ursache können nach wie vor bestehende problematischere Wiederbeschäftigungsmöglichkeiten aufgrund der wirtschaftlichen Situation und der meist größeren Konkurrenz am Arbeitsmarkt in Ostdeutschland sein, die dann besonders bei unfreiwilligen Betriebswechseln negative Folgen haben. Arbeitsplatzwechsel in Regionen

\footnotetext{
${ }^{10}$ Dabei nehmen Relative Risk-Ratios Werte zwischen 0 und unendlich an. Ein Wert von 1 bedeutet, dass das Risiko zwischen den Gruppen nicht differiert, Werte unter eins geben ein geringeres und Werte über eins ein höheres Risiko wieder hinsichtlich des Einkommens ab- bzw. aufgestiegen zu sein.

${ }^{11}$ Mit Ausnahme der regionalen Charakteristika auf Ebene der Raumordnungsregionen bleiben alle übrigen individuellen, betrieblichen und regionalen Merkmale im Modell enthalten. Die übrigen unabhängigen Variablen verändern sich hinsichtlich ihrer Wirkungsrichtung und den Signifikanzen nicht.
} 
Nord- oder Süddeutschlands erfolgen häufiger aufstiegsorientiert. Zudem sind Einkommenserfolge für in Süd- und Norddeutschland Beschäftigte, die innerhalb der Großregion betriebsmobil sind und diejenigen, die die Großregion - unabhängig von der Zielgroßregion - verlassen, wahrscheinlicher als für Erwerbstätige, die ihren Arbeitgeber innerhalb Ostdeutschlands wechseln. Arbeitsortmobilität von Nord- oder Süddeutschland in Richtung Ostdeutschland zieht jedoch ambivalente Einkommenseffekte nach sich, da sie ebenfalls mit einer vergleichsweise höheren Wahrscheinlichkeit von Einkommensverlusten einhergehen kann. ${ }^{12}$

Bezüglich der individuellen Merkmale von Betriebswechslern zeigt sich, dass betriebsmobile Frauen geringere Aufstiegschancen haben als Männer. Ein Grund hierfür könnte sein, dass Frauen überwiegend im öffentlichen Dienst und in breiten kaufmännischen oder in nachgefragten sozialen und Gesundheitsberufen arbeiten, die direkte überbetriebliche Beschäftigungschancen bieten, jedoch auch geringere Aufstiege und Einkommenszuwächse ermöglichen, als dies in männerdominierten, hier besonders technischen Berufen der Fall ist. Wie in Hypothese 5 angenommen, verringert Arbeitsplatzmobilität zwischen Großregionen für Frauen aber zugleich auch die Wahrscheinlichkeit Verluste in Kauf nehmen zu müssen (siehe Tabelle 2, Modell 1a). Auch hier scheinen die überbetrieblichen Beschäftigungschancen in Berufen, in denen Frauen häufiger tätig sind, die Wahrscheinlichkeit von Einkommensverlusten bei Entscheidungen für Betriebswechsel zu mindern.

Mit der Höhe des beruflichen Qualifikationsniveaus reduziert sich die Gefahr Einkommensabstiege zu erfahren. Gleichzeitig erhöhen akademische Abschlüsse die Einkommenserfolgschancen besonders stark (Tabelle 1). Hierbei ist allerdings irrelevant, ob Großregionengrenzen überwunden werden (Tabelle 2, Modell 1b). Akademiker finden auch innerhalb des jeweiligen Landesteils aussichtsreiche Erwerbschancen vor - Hypothese 3 ist abzulehnen.

\section{Etwa hier Tabelle 2: Interaktionseffekte zu Einkommensveränderungen infolge regionaler Mobilitätsprozesse (Multinomiale Schätzmodelle)}

\footnotetext{
12 Berechnungen für westdeutsche Betriebsmobile, die Wechsel in Richtung Ostdeutschland differenziert nach dem beruflichen Qualifikationsniveau betrachten, zeigen, dass insbesondere Geringqualifizierte und ungelernte Beschäftigte, die ihren Arbeitsplatz in Richtung Ostdeutschland verlagern Einkommensverluste hinnehmen müssen, während sich für Erwerbsstätige, die mindestens über einen beruflichen Abschluss verfügen und nach Ostdeutschland wechseln, keine höheren Abstiegswahrscheinlichkeiten nachweisen lassen (Hacket 2009, S. 235).
} 
Mit Blick auf die Altersgruppen wird deutlich, dass Beschäftigte, die 36 Jahre und älter sind, ein vergleichsweise geringeres Einkommensverlustrisiko haben. Sie erzielen aber auch mit einer geringeren Wahrscheinlichkeit Einkommenszuwächse. Ältere Beschäftigte wechseln Großregionen seltener als jüngere. Sie würden weniger lang von einem möglichen Lohnzuwachs profitieren, sie haben zumeist ein höheres spezifisches Humankapital erworben (Mincer 1986) und sind häufig lokal und im Betrieb stärker ökonomisch und sozial verankert. In Tabelle 2 (Modell 1c) zeigt sich, dass die Arbeitsplatzmobilität zwischen Großregionen in der eher kleineren Gruppe von mobilen Erwerbstätigen, die 50 Jahre oder älter sind, die Wahrscheinlichkeit, Einkommenszuwächse zu realisieren, begünstigt. Dies wurde in Hypothese 4 angenommen. Allerdings ist bei einem Wechsel über Großregionengrenzen hinweg bei älteren Erwerbstätigen auch das Risiko von Verlusten größer als bei Jüngeren. Dies deutet auf heterogene Ausgangsbedingungen bzw. Beweggründe räumlicher Mobilität in dieser Altersgruppe hin. Übertritte können aus der Situation eines drohenden Beschäftigungsverlustes oder eines hohen Arbeitslosigkeitsrisikos erfolgen oder ein sehr attraktives Angebot befördert die Arbeitsplatzmobilität. Wechsel aus mittel- und langjährigen Betriebszugehörigkeitsdauern erhöhen vor allem die Wahrscheinlichkeit aufzusteigen. Für Personen, die länger als 7 Jahre im Unternehmen sind, steigt bei einem Betriebswechsel aber auch die Wahrscheinlichkeit von Einkommensverlusten.

Die Relevanz betrieblicher Kontextmerkmale (siehe Kelle (2016)) wird in unserer Analyse deutlich. Demnach sind individuelle Einkommenserfolge nach einem Wechsel in jene Unternehmen wahrscheinlicher, die ein überdurchschnittliches Medianlohnniveau aufweisen. Dies konnte erwartet werden und war entsprechend zu kontrollieren. Mit Blick auf Wirtschaftszweige sehen wir, dass Wechsel in den Wirtschaftszweig unternehmensnahe Dienstleistungen, der insbesondere auch die Leiharbeit einschließt, mit erhöhter Wahrscheinlichkeit von Einkommensverlusten einhergehen.

Bezüglich der regionalen Strukturindikatoren war angenommen worden, dass zentrale Räume Agglomerationsvorteile bieten. Die Annahmen basieren auf dem dualen Raumkonzept (Fassmann/Meusberger 1997), dem Zentrum-Peripherie-Modell (Krugman 1991) und suchund matchingtheoretischen Ansätzen (Wheeler 2001, 2006) und konnten teilweise empirisch belegt werden (Glaeser/Maré 2001). Entgegen der Hypothese 2(a) zeigen unsere Ergebnisse, dass Arbeitgeberwechsel in agglomerierte Zielräume, bereinigt um das regionale Preisniveau, unmittelbar keine Einkommenszuwächse nach sich ziehen. Allerdings verringern sie ebenso Einkommensverluste (Tabelle 2, Modell 1d), so dass Hypothese 2(b) bestätigt werden kann. Für Zielregionen mit Verstädterungstendenzen lässt sich ein einkommenserfolgsförderlicher Effekt feststellen. Dass die Beschäftigungsoptionen in weniger dicht besiedelten Regionen ebenfalls vielfältig sind, legen die auch außerhalb von Agglomerationsräumen nicht geringeren 
Beschäftigtenquoten nahe (Statistisches Bundesamt 2016, S. 356). Diese wären dann, unseren Ergebnissen zufolge mit aussichtsreichen Einkommenschancen verbunden und ein Indiz für die dynamische Entwicklung von Beschäftigungsmöglichkeiten im Umland von Städten (Hilal et al. 2017).

Wirtschaftliche Prosperitätsindikatoren und die Arbeitsmarktlage der Zielregion sind für die individuellen Einkommenschancen - entgegen unserer Vermutung - wenig bedeutsam. Ein höheres lokales Bruttoinlandsprodukt, d. h. hohe Wirtschaftskraft und damit höhere Preise im Zielraum begünstigen direkt nach einem Wechsel weder Einkommenserfolge noch verringern sie Einkommensverlustrisiken. Die Arbeitsmarktlage, die Humankapitalausstattung und das Wohlstandsniveau der Zielraumordnungsregionen beeinflussen weder die Aufstiegs- noch die Abstiegschancen statistisch signifikant, sofern geografische, individuelle und betriebliche Aspekte simultan berücksichtigt werden.

Erwartungsgemäß zeichnen sich kaum konjunkturbedingte Erfolgsmuster betrieblicher Mobilitätsprozesse ab. Erkennbar ist jedoch, dass die Jahre 2005 bis 2010 Einkommensaufstiege der Betriebsmobilen fördern. Interessanterweise hemmt der konjunkturelle Einbruch durch die Weltwirtschaftskrise 2009 die Erfolgswahrscheinlichkeit von Einkommenssteigerungen nicht. Selbst die Wahrscheinlichkeit von Einkommensabstiegen reduziert sich im Krisenjahr 2009, wie zu Beginn des Analysezeitraumes im Jahr 2001, das noch von der Dotcom-Krise beeinflusst war. Dies deutet auf eine positive Selektion derjenigen Beschäftigten hin, die unter diesen gesamtwirtschaftlichen Rahmenbedingungen betriebsmobil sind.

\section{Mobilität zwischen Großregionen. Diskussion und Fazit}

Unter Berücksichtigung der raum- und wirtschaftsstrukturellen Disparitäten zwischen Ost-, Nord-, und Süddeutschland und der Wanderungsrichtung untersucht der Beitrag die Einflussfaktoren von substantiellen Einkommensveränderungen. Betrachtet werden hierbei zugleich Individualmerkmale sowie betriebliche und regionale Charakteristika des Zielbetriebskontextes.

Insgesamt zeigen unsere Ergebnisse, jüngere und hochqualifizierte Beschäftigte realisieren bei Arbeitsplatzwechseln über Großregionsgrenzen hinweg mit einer vergleichsweise höheren Wahrscheinlichkeit substantielle Einkommenszuwächse. Zudem sind für alle Wanderungsrichtungen mit einer hohen Wahrscheinlichkeit Einkommenszuwächse verbunden. Aussichtsreichere Einkommensperspektiven außerhalb Ostdeutschlands und die ebenfalls festzustellenden Abstiegsrisiken bei Mobilität von Nord- und Süddeutschland in Richtung Osten sprechen dafür, dass die Beschäftigungsbedingungen zwischen den 
Großregionen bzw. zwischen Ost- und Westdeutschland ungleich verteilt sind. Damit geht die Gefahr einher, dass sich die Strukturschwäche Ostdeutschlands weiterhin und dauerhaft reproduziert. Unattraktivere Einkommens- und Beschäftigungsperspektiven können weiterhin Abwanderung besonders auch von jüngeren und besser qualifizierten Personengruppen in prosperierende Räume befördern (Ganesch et al. 2017; Schlömer 2004; Seibert 2008, Shapiro 2006). Folgt man der These, dass die Anteile junger Erwerbspersonen als Indikator für das zukünftige wirtschaftliche Leistungs-, Innovations- und Wachstumspotential gelten (Ryder 1965; Niedomysl/Hansen 2010; Fink/Thiemann 2016), dann mindern derartige Alterskonstellationen die Standortattraktivität mittel- und langfristig.

Unsere Ergebnisse stützen damit im Grundsatz nutzentheoretische Erklärungsansätze auf Basis des place-utility-Ansatz (Wolpert 1965) sowie suchtheoretischer Überlegungen (McKenna 1985; Kalter 1994). Allerdings gibt es auch einzelne Ausnahmen. So finden sich teilweise Risiken von Einkommenseinbußen. Diese gelten einerseits für Wechsel in Richtung Ostdeutschland. Ungleichheiten der Einkommenschancen bestehen bei Bereinigung regionaler Preisunterschiede auch lange nach der Wiedervereinigung fort. Die Wahrscheinlichkeit von Einkommensverlusten besteht zudem für einen Teil der vergleichsweise mobilen jüngeren Beschäftigten, für geringer Qualifizierte, für Ausländer sowie für Personen, die schon länger in Unternehmen beschäftigt sind und besonders deutlich für Personen, die im Bereich unternehmensnaher Dienstleistungen tätig sind (und hier vermutlich insbesondere in den vielen Leiharbeitsunternehmen dieses Wirtschaftszweiges).

Interessant ist und nicht zu erwarten war, dass Wechsel in wirtschaftlich leistungsstarke Zielräume, gemessen am Bruttoinlandsprodukt der jeweiligen Raumordnungsregion und bei Kontrolle auch betrieblicher Faktoren die Wahrscheinlichkeit von Einkommenserfolge mindern. Es sind damit das eigene (verhandelte bzw. akzeptierte) Lohniveau sowie der betriebliche Kontext der durchschnittlichen Lohnhöhe in der Betriebsstätte bedeutsamer für Einkommenszuwächse nach Mobilität, als das wirtschaftsstrukturelle Umfeld der gewählten Zielregion. Allerdings ist hierbei wiederum zu berücksichtigen, dass Einkommenseffekte unmittelbar nach dem Betriebswechsel betrachtet wurden. Möglicherweise treten positive Effekte (die ggf. beim Zuzug in urbane Räume erwartet wurden) später ein.

Deutlich wurde, dass Mobilitätsbewegungen über große Distanzen hinweg bestehen, die unmittelbar nicht zu Einkommenszuwächsen führen und einem eng verstandenem ökonomischen Kalkül entgegenstehen. Eine Aufklärung hierüber ist auf eine umfänglichere Berücksichtigung von Rahmenbedingungen angewiesen, die etwa familiäre und soziale Kontexte sowie ortsgebundene Ressourcen mit einbezieht. Dies lässt die Datenlage insgesamt nicht zu (Struck/Ganesch 2018). Und so können diese Aspekte auch in der hier 
verwendeten Datenstruktur von verknüpften Erwerbs-, Betriebs- und Regionaldaten, die ihre Stärke in der präzisen Erfassung von Beschäftigungszeiten und Erwerbseinkommen im betrieblichen und regionalwirtschaftlichen Kontext hat, nicht kontrolliert werden. 


\section{Literaturverzeichnis}

Albrech, J./Fink, P./Tiemann, H. (Hrsg.) (2016): Ungleiches Deutschland: Sozioökonomischer Disparitätsbericht 2015, Bonn.

Angrist, J.D./Pischke, J.S. (2009): Mostly Harmless Econometrics: An Empiricist's Companion, Princeton.

Arntz, M./Gregory, T./Lehmer, F. (2014): Can Regional Employment Disparities Explain the Allocation of Human Capital Across Space?, Regional Studies, 48, S.1719-1738.

Baron, J.N./Bielby, W.T. (1980): Bringing the firms back in: stratification, segmentation, and the organization of work, American Sociological Review, 45, S.737-765.

Blien, U./Kirchhof, K./Ludewig, O. (2006): Agglomeration effects on labour demand, IAB Discussion Paper No. 28.

Blien, U./Wolf, K. (2002): Regional development of employment in eastern Germany: an analysis with an econometric analogue to shift-share techniques, Papers in Regional Science, 81, S. 391-414.

Boyle, P./Halfaree, K./Robinson, V. (1998): Exploring contemporary migration, Harlow, Essex.

Buch, T. (2006): Regionale Mobilität auf dem deutschen Arbeitsmarkt: Eine theoretische und empirische Analyse regionaler Mismatcharbeitslosigkeit in Zeiten der Hartz-Reformen, Hamburg.

Buch, T./Hamann, S., Niebuhr, A./Rossen, A. (2014): How to Woo the Smart Ones? Evaluating the Determinants that Particularly Attract Highly Qualified People to Cities, HWWI Research Paper, Hamburg Institute of International Economics.

Bundesinstitut für Bau-, Stadt- und Raumforschung (2016): Laufende Raumbeobachtung - Raumabgrenzungen, http://www.bbsr.bund.de/BBSR/DE/Raumbeobachtung/Raumabgrenzungen/Raumordnungsregionen/raumordnun gsregionen_node.html [06.02.2019].

Damelang, A. (2007): Räumliche Mobilität von türkischen Arbeitnehmern * eine Analyse mit der IABBeschäftigtenstichprobe 2001, IAB Discussion Paper, 21.

Eichhorst, W./Stephan, G./Struck, O. (2017): Struktur und Ausgleich des Arbeitsmarktes, working paper Forschungsförderung, Hans-Böckler-Stiftung, Nr. 28.

Farhauer, O./Granato, N. (2006): Regionale Arbeitsmärkte in Westdeutschland: Standortfaktoren und Branchenmix entscheidend für Beschäftigung * eine vergleichende Länderstudie zeigt Vor- und Nachteile von Regionen und liefert erste Hinweise auf Handlungspotenziale, IAB-Kurzbericht, No. 4.

Fassmann, H./Meusburger, P. (1997): Arbeitsmarktgeographie. Erwerbstätigkeit und Arbeitslosigkeit im räumlichen Kontext, Stuttgart.

Fink, P./Tiemann, H. (2016): Deutschland driftet auseinander, in: Albrech,J./P. Fink /H. Tiemann (Hrsg.), Ungleiches Deutschland: Sozioökonomischer Disparitätsbericht 2015, Ungleiches Deutschland, Bonn.

Ganesch, F. (2018): Regional Mobility and Spatial Inequality: Determinants of Spatial Labor Market Behavior Considering Firm- and Region-Specific Factors. Spatial Research and Planning 76, 497-514

Ganesch, F./Dütsch, M./Struck, O. (2017): Räumliche Mobilität am Arbeitsmarkt: Einfluss- und Erfolgsfaktoren, Working Paper der Professur für Arbeitswissenschaft der Universität Bamberg, Nr. 21.

Giesecke, J./Heisig, J.P. (2010): Destabilisierung und Destandardisierung, aber für wen? Die Entwicklung der westdeutschen Arbeitsplatzmobilität seit 1984, Kölner Zeitschrift für Soziologie und Sozialpsychologie, 62, S. 403-435.

Glaeser, E.L./Maré, D.C. (2001): Cities and Skills, Journal of Labor Economics, 19, S. 316-342.

Granato, N./Haas, A./Hamann, S./Niebuhr, A. (2009): Arbeitskräftemobilität in Deutschland Qualifikationsspezifische Befunde regionaler Wanderungs- und Pendlerströme, Raumforschung und Raumordnung, 67, S. 21-33.

Grotheer, M., /Struck, O. (2003): Beschäftigungsstabilität: Entwicklung und Arbeitszufriedenheit. Ergebnisse aus der IAB-Beschäftigtenstichprobe 1975-1997 und der BIBB/IAB-Erhebung, Mitteilungen aus der Arbeitsmarkt- und Berufsforschung, 36, S. 300-328.

Haas, A. (2000): Arbeitsmarktausgleich: Regionale Mobilität gestiegen; Bei einem Betriebswechsel werden immer öfter größere Entfernungen überwunden - gerade auch von Arbeitslosen, IAB-Kurzbericht, Institut für Arbeitsmarkt- und Berufsforschung (IAB) der Bundesagentur für Arbeit. Nr. 4.

Hacket, A. (2009): Lohnt sich Mobilität? Einkommensperspektiven in internen und externen Arbeitsmärkten in den ersten Berufsjahren, Wiesbaden.

Hausmann, A.-C./Kleinert, C. (2014): Berufliche Segregation auf dem Arbeitsmarkt: Männer- und Frauendomänen kaum verändert, IAB-Kurzbericht, Institut für Arbeitsmarkt- und Berufsforschung (IAB) der Bundesagentur für Arbeit, Nr. 9. 
Hendry, C. (2003): Applying employment systems theory to the analysis of national models of HRM. The International Journal of Human Resource Management, 14, S. 1430-1442.

Hilal, M./Legras, S./Cavailhès, J. (2017): Peri-Urbanisation: Between Residential Preferences and Job Opportunities. Raumforschung und Raumordnung, S. 1-15.

Hillmert, S. (2006): Mobilität am Arbeitsmarkt und regionale Disparitäten, in: Rehberg, K.-S. (Hrsg.); Deutsche Gesellschaft für Soziologie (DGS) (Hrsg.): Soziale Ungleichheit, kulturelle Unterschiede: Verhandlungen des 32 , Kongresses der Deutschen Gesellschaft für Soziologie in München, Teilbd. 1 und 2, Frankfurt am Main, S. 33913401.

Hinz, T./Abraham, M. (2008): Theorien des Arbeitsmarktes: Ein Überblick, in: Abraham M./Hinz, T. (Hrsg.), Arbeitsmarktsoziologie: Probleme, Theorien, empirische Befunde, Wiesbaden, S. 17-68.

Hinz, T./Gartner, H. (2005): Geschlechtsspezifische Lohnunterschiede in Branchen, Berufen und Betrieben. Zeitschrift für Soziologie, 34, S. 22-39.

Huinink, J./Kley, S. (2008): Regionaler Kontext und Migrationsentscheidungen im Lebensverlauf. Kölner Zeitschrift für Soziologie und Sozialpsychologie 48(8):163-184.

Hunt, J. (2006): Staunching Emigration from East Germany: Age and the Determinants of Migration, Journal of the European Economic Association, 4-5, S. 1014-1037.

Jacobebbinghaus, P. (2008): LIAB-Datenhandbuch, Version 3.0. FDZ-Datenreport, Bundesagentur für Arbeit, Nr. 3.

Johnson, W.R. (1978): A Theory of Job Shopping, Quarterly Journal of Economics, 97, S. 261-277.

Jovanovic, B. (1979): Job Matching and the Theory of Turnover, Journal of Political Economy, 87, S. 972-990.

Kalter, F. (1994): Pendeln statt Migration? Die Wahl und Stabilität von Wohnort-Arbeitsort-Kombinationen, Zeitschrift für Soziologie, 23, S. 460-476.

Novemsky N./ Kahneman, D. (2005). The Boundaries of Loss Aversion. Journal of Marketing Research: 42 (2):119-128.

Kawka, R. (2009): Regionaler Preisindex. Berichte / Bundesamt für Bauwesen und Raumordnung, BBSR, Nr. 30.

Kelle, M. (2016): Wage growth, urbanization, and firm characteristics: Evidence for Germany, Ruhr Economic papers, Nr. 631.

Kézdi, G. (2004): Robust Standard Error Estimation in Fixed-Effects Panel Models, Hungarian Statistical Review, 89, S. 96-116.

Kratz, F./Brüderl, J. (2013): Returns to Regional Migration: Causal Effect or Selection on Wage Growth?, Schmollers Jahrbuch, 133, S. 227-238.

Krugman, P.R. (1991): Geography and trade. Leuven, Belgium, Cambridge, u.a.

Lazear, E.P. (1981): Agency, earning profiles, productivity and hours restrictions. American Economic Review, 71, S. $606-620$.

Lehmer, F./Ludsteck, J. (2011): The returns to job mobility and inter-regional migration: Evidence from Germany*, Papers in Regional Science, 90, S.549-572.

Lehmer, F./Möller, J. (2008): Group-specific effects of interregional mobility on earnings - a microdata analysis for Germany. Regional Studies, 42, S. 657-673.

Lepak, D.P./Liao, H./Chung, Y./Harden, E.E. (2006): A conceptual review of human resource management systems in strategic human resource management research, Research in personnel and human resources management, 25, S. 217-271.

Maier, M.F./Sprietsma, M. (2016): Does it pay to move? Returns to regional mobility at the start of the career for tertiary education graduates, ZEW Discussion Paper, No. 16-060.

McKenna, C.J. (1985): Uncertainty and the labour market: Recent developments in job-search theory. Brighton, Sussex.

Mertens, A./Haas, A. (2006): Regionale Arbeitslosigkeit und Arbeitsplatzwechsel in Deutschland - Eine Analyse auf Kreisebene, Jahrbuch für Regionalwissenschaft, 26, S. 147-169.

Meusburger, P. (1998): Bildungsgeographie: Wissen und Ausbildung in der räumlichen Dimension, Heidelberg.

Mincer, J. (1986): Wage Changes in Job Changes, in: Ehrenberg, R.E. (Hrsg.), Research. Labor Economics, S.171-197.

Niedomysl, T./Hansen, H.K. (2010): What matters more for the decision to move: jobs versus amenities, Environment and Planning, 42, S. 1636-1649. 
OECD (2008): Education at a glance 2008, OECD INDICATORS, https://www.oecd.org/education/skills-beyondschool/41284038.pdf [31.01.2019].

Reichelt, M./Abraham, M. (2015): Occupational and Regional Mobility as Substitutes: A New Approach to Understanding Job Changes and Wage Inequality, Social Forces, 95, S. 1-27.

Rendtel, U./Schwarze, J. (1996): Schätzungen von Lohnkurven für Westdeutschland mit einem verallgemeinerten Varianz-Komponenten-Modell, Mitteilungen aus der Arbeitsmarkt- und Berufsforschung, 29, S. 491-493.

Ruppenthal, S. (2010): Vielfalt und Verbreitung berufsbedingter räumlicher Mobilität im europäischen Vergleich, Bevölkerungsforschung Aktuell, 31, S. 2-7.

Ryder, N.B. (1965): The Cohort as a Concept in the Study of Social Change, American Sociological Review, 30, S. $843-861$.

Sander, N. (2014): Internal Migration in Germany, 1995-2010: New Insights into East-West Migration and Reurbanisation, Comparative Population Studies, 39, S. 217-246.

Schlitte, F./Böttcher, F./Niebuhr, A./Revilla Diez, J. (2010): The determinants of regional disparities in skill segregation - Evidence from a cross section of German regions. HWWI Research Paper. Hamburg Institute of International Economics (HWWI), No., S. 1-36.

Schlömer, C. (2004): Binnenwanderungen seit der deutschen Einigung. Raumforschung und Raumordnung, 62 , S. $96-108$.

Seibert, H. (2008): Räumliche Mobilität als Notwendigkeit zur Vermeidung von Arbeitslosigkeit am Beispiel der Region Berlin-Brandenburg, in: Barlösius, E./Neu, C. (Hrsg.), Peripherisierung - eine neue Form sozialer Ungleichheit, Berlin, S. 37-46.

Shapiro, J. (2006): Smart Cities: Quality of Life, Productivity, and the Growth Effects of Human Capital, The Review of Economics and Statistics, 88, S. 324-335.

Sjaastad, L.A. (1962). The Costs and Returns of Human Migration, Journal of Political Economy, 70, S. 80-93.

Statistisches Bundesamt (2015): 25 Jahre Deutsche Einheit, Wiesbaden.

- (2016): Datenreport 2016: Ein Sozialbericht für die Bundesrepublik Deutschland, Bonn.

- (2017): Verdienste auf einen Blick,

https://www.destatis.de/DE/Publikationen/Thematisch/VerdiensteArbeitskosten/Arbeitnehmerverdienste/Broschue reVerdiensteBlick0160013179004.pdf [06.02.2019].

Struck, O. (2006): Flexibilität und Sicherheit: Empirische Befunde, theoretische Konzepte und institutionelle Gestaltung von Beschäftigungsstabilität, Wiesbaden.

- (2017): Aufschwung und Unzufriedenheit: Strukturwandel und Lebenssituation in Ostdeutschland, Working Paper der Professur für Arbeitswissenschaft der Universität Bamberg, Nr. 19.

Struck, O./Grotheer, M./Köhler, C./Schröder, T. (2007): Instabile Beschäftigung. Neue Ergebnisse zu einer alten Kontroverse, Kölner Zeitschrift für Soziologie und Sozialpsychologie, 59, S. 294-317.

Struck, O./Ganesch, F. (2018): Herausforderungen an Datenstrukturen in der Arbeits- und Arbeitsmarktforschung, WSI-Mitteilungen, No. 71.

Südekum, J. (2008): Convergence of the skill composition across German regions, Regional Science and Urban Economics, 38, S. 148-159.

Wheeler, C. (2001): Search, Sorting, and Urban Agglomeration, Journal of Labor Economics, 19, S. 879-899.

- (2006): Cities and the growth of wages among young workers: Evidence from the NLSY, Journal of Urban Economics, 60, S. 162-184.

Windzio, M. (2004a): Kann der regionale Kontext zur „Arbeitslosenfalle“ werden? Der Einfluss der Arbeitslosigkeit auf die Mobilität zwischen regionalen Arbeitsmärkten in Westdeutschland, Kölner Zeitschrift für Soziologie und Sozialpsychologie, 56, S. 257-278.

- (2004b): Zwischen Nord- und Süddeutschland: Die Überwindung räumlicher Distanzen bei der Arbeitsmarktmobilität, Zeitschrift für ArbeitsmarktForschung, 37, S. 29-44.

- (2013): Räumliche Mobilität, in: Mau, S./Schöneck, N.M. (Hrsg.), Handwörterbuch zur Gesellschaft Deutschlands, Wiesbaden, S. 664-675.

Windzio, M./Huinink, J. (2010): Migration, regionale Entwicklung und die Integration von Zuwanderern. Eine kurze Einführung, in: Wolfgang-Ritter-Stiftung (Hrsg.), Migration und regionale Entwicklung, Oldenburg, S. 9-19.

Wirtschafts- und Sozialwissenschaftliches Institut der Hans-Böckler-Stiftung (WSI) (2018): Lohnspiegel, https://www.lohnspiegel.de/html/index.php [01.03.2018].

Wolpert, J. (1965): Behavioral aspects of the decision to migrate, Papers in Regional Science, 15, S. 159-169. 


\section{Anhang}

Anhang 1: Arbeitslosigkeitsquoten auf der Ebene von Raumordnungsregion im Jahr 2010

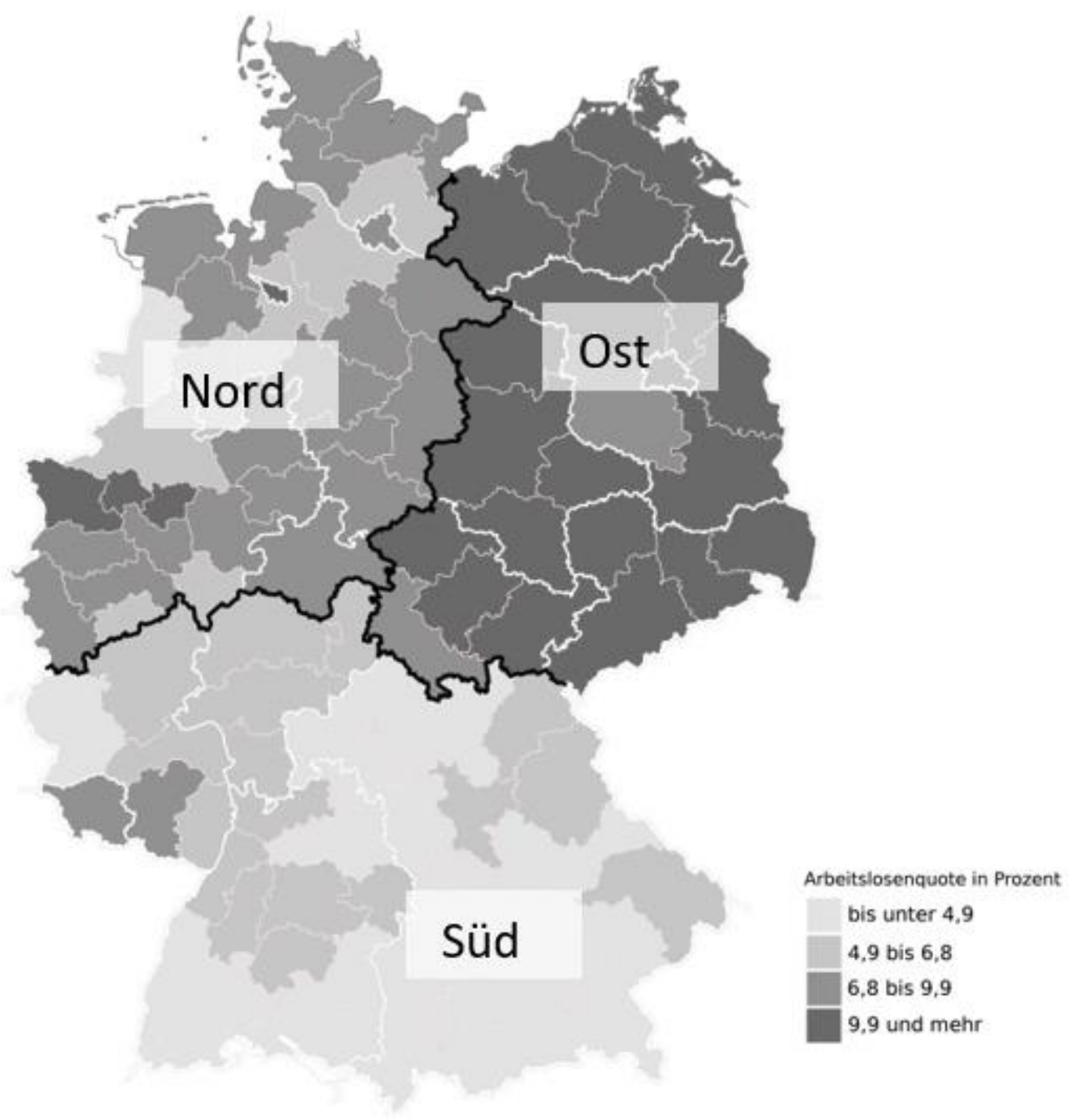

*Anmerkung: Die Arbeitslosigkeitsquoten wurden auf der Ebene von Raumordnungsregionen gemessen. Die gewählten Analysekategorien ergeben sich aus der Quartilsverteilung der beobachteten lokalen Arbeitslosigkeitsquoten. Die schwarze Linie kennzeichnet die verwendete Großregionendefinition, die die Großregionen Nord, Süd und Ost unterscheidet. 
Anhang 2: Deskription Analysesample Betriebsmobile

\begin{tabular}{|l|c|c|c|c|}
\hline & & \multicolumn{3}{|c|}{ Mobilität } \\
\cline { 2 - 5 } & & $\begin{array}{c}\text { zwischen } \\
\text { Groß- } \\
\text { regionen }\end{array}$ & $\begin{array}{c}\text { zw. Raum- } \\
\text { ordnungs- } \\
\text { regionen }\end{array}$ & $\begin{array}{c}\text { keine } \\
\text { räumliche } \\
\text { Mobilität }\end{array}$ \\
\hline \hline Insgesamt & 184785 & 15974 & 57518 & 127267 \\
$6,6 \%$ & $31,1 \%$ & $68,9 \%$ \\
\hline \hline Geschlecht & & & & \\
\hline weiblich & 56397 & 4469 & 16009 & 40388 \\
\hline männlich & $100,0 \%$ & $7,9 \%$ & $28,4 \%$ & $71,6 \%$ \\
\hline \hline Berufliche Qualifikation & 128388 & 11505 & 41509 & 86879 \\
\hline Ungelernt & 31131 & 1940 & $32,3 \%$ & $67,7 \%$ \\
\hline Berufliche & $100,0 \%$ & $6,2 \%$ & $27,0 \%$ & $73,0 \%$ \\
Ausbildung & 131405 & 10498 & 38461 & 92944 \\
\hline Hochschul- & $100,0 \%$ & $8,0 \%$ & $29,3 \%$ & $70,7 \%$ \\
abschluss & 22249 & 3536 & 10666 & 11583 \\
\hline \hline Altersgruppen & $100,0 \%$ & $15,9 \%$ & $47,9 \%$ & $52,1 \%$ \\
\hline 18 bis 35- & 97786 & 9548 & 33400 & 64386 \\
Jährige & $100,0 \%$ & $9,8 \%$ & $34,2 \%$ & $65,8 \%$ \\
\hline 36 bis 50- & 65529 & 5320 & 19650 & 45879 \\
Jährige & $100,0 \%$ & $8,1 \%$ & $30,0 \%$ & $70,0 \%$ \\
\hline 51 bis 65- & 21470 & 1106 & 4468 & 17002 \\
Jährige & $100,0 \%$ & $5,2 \%$ & $20,8 \%$ & $79,2 \%$ \\
\hline \hline
\end{tabular}


Tabelle 1: Einkommensveränderungen infolge regionaler Mobilitätsprozesse (multinomiale Schätzmodelle) ${ }^{13}$

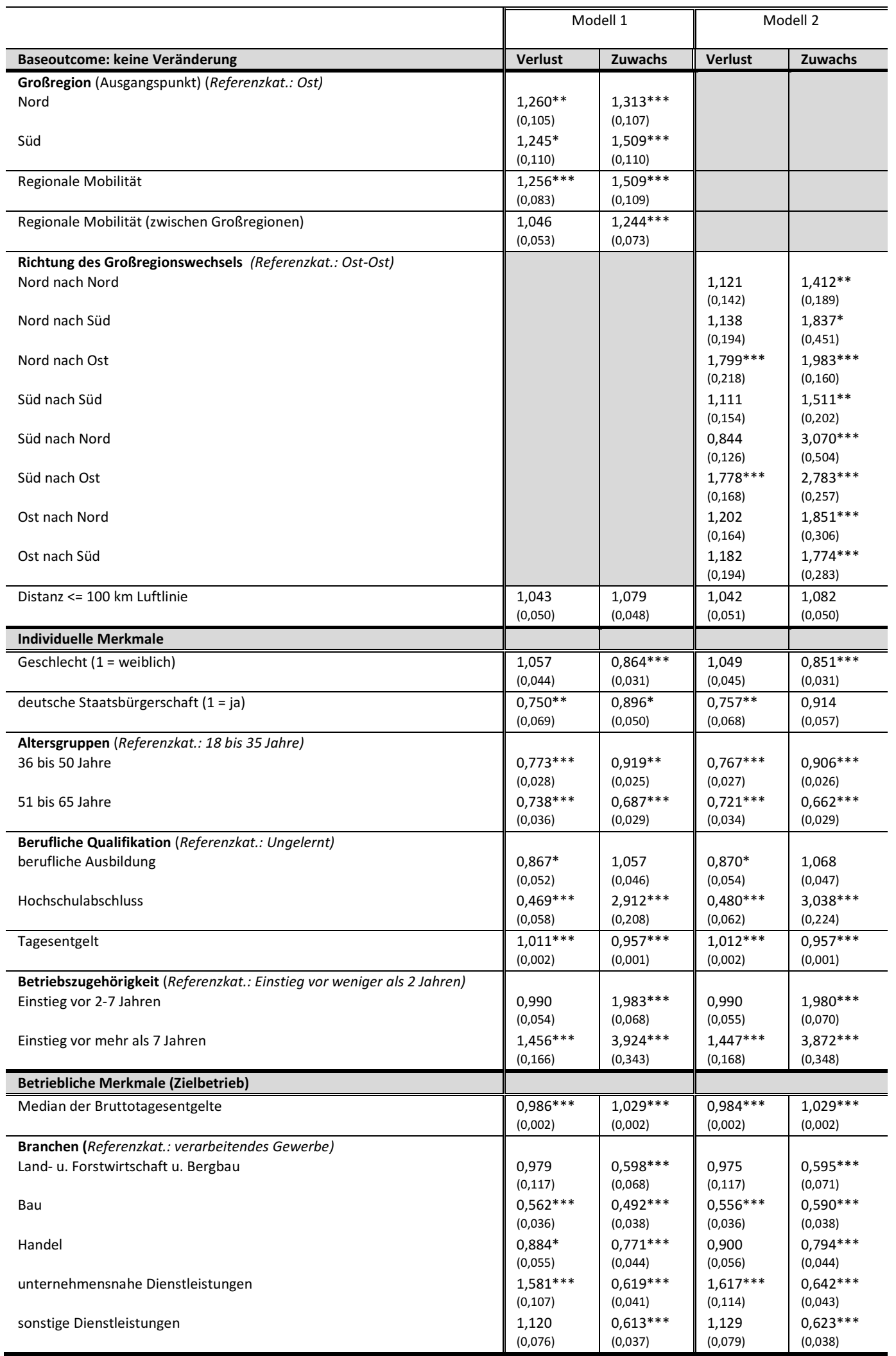

${ }^{13}$ lokale Preisunterschiede werden berücksichtigt 


\begin{tabular}{|c|c|c|c|c|}
\hline \multicolumn{5}{|l|}{ Regionale Strukturmerkmale (Zielregion) } \\
\hline $\begin{array}{l}\text { Regionstyp (Referenzkat.: ländliche Region) } \\
\text { städtische Region }\end{array}$ & $\begin{array}{l}1,141 \\
(0,107)\end{array}$ & $\begin{array}{l}1,262 \\
(0,164)\end{array}$ & $\begin{array}{l}1,195 \\
(0,118)\end{array}$ & $\begin{array}{l}1,215 \\
(0,153)\end{array}$ \\
\hline Region mit Verstädterungstendenz & $\begin{array}{l}1,083 \\
(0,091)\end{array}$ & $\begin{array}{l}1,171^{*} \\
(0,086)\end{array}$ & $\begin{array}{l}1,116 \\
(0,101)\end{array}$ & $\begin{array}{l}1,162^{*} \\
(0,085)\end{array}$ \\
\hline Arbeitslosenquote & $\begin{array}{l}1,005 \\
(0,009)\end{array}$ & $\begin{array}{l}1,009 \\
(0,008)\end{array}$ & $\begin{array}{l}0,998 \\
(0,012)\end{array}$ & $\begin{array}{l}1,007 \\
(0,009)\end{array}$ \\
\hline Hochqualifizierten-Beschäftigtenquote & $\begin{array}{l}1,027 \\
(0,023) \\
\end{array}$ & $\begin{array}{l}0,993 \\
(0,022) \\
\end{array}$ & $\begin{array}{l}1,008 \\
(0,022) \\
\end{array}$ & $\begin{array}{l}1,003 \\
(0,025) \\
\end{array}$ \\
\hline BIP je Einwohner & $\begin{array}{l}1,003 \\
(0,006)\end{array}$ & $\begin{array}{l}0,985^{*} \\
(0,006)\end{array}$ & $\begin{array}{l}1,008 \\
(0,007)\end{array}$ & $\begin{array}{l}0,982^{* *} \\
(0,006)\end{array}$ \\
\hline \multicolumn{5}{|l|}{ Konjunkturelle Gesamtlage } \\
\hline \multicolumn{5}{|l|}{ Jahre (Referenzkat.: 2000) } \\
\hline 2001 & $\begin{array}{l}0,882^{*} \\
(0,051)\end{array}$ & $\begin{array}{l}0,936 \\
(0,052)\end{array}$ & $\begin{array}{l}0,878^{*} \\
(0,051)\end{array}$ & $\begin{array}{l}0,934 \\
(0,052)\end{array}$ \\
\hline 2002 & $\begin{array}{l}0,889 \\
(0,062)\end{array}$ & $\begin{array}{l}0,925 \\
(0,049)\end{array}$ & $\begin{array}{l}0,889 \\
(0,064)\end{array}$ & $\begin{array}{l}0,924 \\
(0,046)\end{array}$ \\
\hline 2003 & $\begin{array}{l}0,982 \\
(0,070)\end{array}$ & $\begin{array}{l}0,942 \\
(0,069)\end{array}$ & $\begin{array}{l}0,983 \\
(0,071)\end{array}$ & $\begin{array}{l}0,938 \\
(0,069)\end{array}$ \\
\hline 2004 & $\begin{array}{l}0,798^{* *} \\
(0,068)\end{array}$ & $\begin{array}{l}1,004 \\
(0,079)\end{array}$ & $\begin{array}{l}0,800^{* *} \\
(0,069)\end{array}$ & $\begin{array}{l}1,010 \\
(0,079)\end{array}$ \\
\hline 2005 & $\begin{array}{l}1,002 \\
(0,069)\end{array}$ & $\begin{array}{l}1,179^{*} \\
(0,080)\end{array}$ & $\begin{array}{l}1,010 \\
(0,072)\end{array}$ & $\begin{array}{l}1,190^{*} \\
(0,082)\end{array}$ \\
\hline 2006 & $\begin{array}{l}0,930 \\
(0,057)\end{array}$ & $\begin{array}{l}1,308^{* * *} \\
(0,070)\end{array}$ & $\begin{array}{l}0,931 \\
(0,060)\end{array}$ & $\begin{array}{l}1,326^{* * *} \\
(0,073)\end{array}$ \\
\hline 2007 & $\begin{array}{l}0,971 \\
(0,080)\end{array}$ & $\begin{array}{l}1,598^{* * *} \\
(0,105)\end{array}$ & $\begin{array}{l}0,952 \\
(0,078)\end{array}$ & $\begin{array}{l}1,605^{* * *} \\
(0,112)\end{array}$ \\
\hline 2008 & $\begin{array}{l}0,930 \\
(0,051)\end{array}$ & $\begin{array}{l}2,087^{* * *} \\
(0,117)\end{array}$ & $\begin{array}{l}0,905 \\
(0,050)\end{array}$ & $\begin{array}{l}2,081^{* * *} \\
(0,125)\end{array}$ \\
\hline 2009 & $\begin{array}{l}0,829 * \\
(0,068)\end{array}$ & $\begin{array}{l}1,821^{* * *} \\
(0,132)\end{array}$ & $\begin{array}{l}0,805^{* *} \\
(0,068)\end{array}$ & $\begin{array}{l}1,787^{* * *} \\
(0,135)\end{array}$ \\
\hline 2010 & $\begin{array}{l}1,027 \\
(0,089)\end{array}$ & $\begin{array}{l}2,487 * * * \\
(0,228)\end{array}$ & $\begin{array}{l}0,977 \\
(0,091) \\
\end{array}$ & $\begin{array}{l}2,380 * * * \\
(0,212)\end{array}$ \\
\hline Beobachtungen & \multicolumn{2}{|c|}{184,785} & \multicolumn{2}{|c|}{184,785} \\
\hline Wald-Chi2 & \multicolumn{2}{|c|}{$71.769,43$} & \multicolumn{2}{|c|}{$67.227,74$} \\
\hline Prob> Chi2 & \multicolumn{2}{|c|}{0.0000} & \multicolumn{2}{|c|}{0.0000} \\
\hline Pseudo R2 & \multicolumn{2}{|c|}{0,1670} & \multicolumn{2}{|c|}{0,1659} \\
\hline Log-Likelihood (final value) & \multicolumn{2}{|c|}{$-161.951,71$} & \multicolumn{2}{|c|}{$-162.169,98$} \\
\hline
\end{tabular}

***/**/* bezeichnet die statistische Signifikanz auf dem 0,001/0,01/0,05 Niveau

multinomiales Regressionsmodell mit Angabe von Relativ-Risk-Ratios, clusterrobuste Standardfehler auf Ebene der Raumordnungsregionen;

Kontrolliert ist zudem das Arbeitnehmerentgelt der jeweiligen Raumordnungsregion;

Quelle: LIAB (LM 9310), eigene Berechnungen

Tabelle 2: Interaktionseffekte zu Einkommensveränderungen infolge regionaler Mobilitätsprozesse (Multinomiale Schätzmodelle)

\begin{tabular}{|c|c|c|}
\hline \multicolumn{3}{|l|}{ Ergänzung Interaktionsterme } \\
\hline & Verlust & Zuwachs \\
\hline \multicolumn{3}{|l|}{ Modell 1a: Geschlecht X Großregionenmobilität } \\
\hline Frauen X Großregionenmobilität & $0,822 * *$ & $0,822^{* *}$ \\
\hline \multicolumn{3}{|c|}{$\begin{array}{l}\text { Modell 1b: Qualifikationsgruppen X Großregionenmobilität (Basis: kein beruflicher Abschluss und keine } \\
\text { Großregionenmobilität) }\end{array}$} \\
\hline berufliche Ausbildung $\mathrm{X}$ Großregionenmobilität & 1,157 & 1,134 \\
\hline Hochschulabschluss $X$ Großregionenmobilität & 0,814 & 1,049 \\
\hline \multicolumn{3}{|c|}{ Modell 1c: Altersgruppen X Großregionenmobilität (Basis: Alter < 36 und keine Großregionenmobilität) } \\
\hline 36-50 Jahre $X$ Großregionenmobilität & $1,135^{*}$ & $1,205^{*}$ \\
\hline 51-65 Jahre $X$ Großregionenmobilität & $1,347^{* *}$ & $1,439^{* *}$ \\
\hline
\end{tabular}




\begin{tabular}{|c|c|c|}
\hline \multicolumn{3}{|c|}{ Modell 1d: Regionstyp X Großregionenwechsel (ländliche Region und keine Großregionenmobilität) } \\
\hline städtische Region X Großregionenwechsel & $0,733^{*}$ & $0,717^{*}$ \\
\hline Region mit Verstädterungstendenz $X$ Großregionenwechsel & 0,821 & 0,841 \\
\hline
\end{tabular}

Die Modellschätzungen 1a-d berücksichtigen neben den hier dokumentierten Interaktionstermen die in Modell 1 dargestellten Variablen. ***/**/* bezeichnet die statistische Signifikanz auf dem 0,001/0,01/0,05 Niveau

multinomiales Regressionsmodell mit Angabe von Relativ-Risk-Ratios, clusterrobuste Standardfehler auf Ebene der Raumordnungsregionen Quelle: LIAB (LM 9310), eigene Berechnungen 


\section{Erklärung zur bisherigen Publikation von Fachartikeln der Disserta- tion}

Employment trajectories in heterogeneous regions: Evidence from Germany, in: Advances in Life Course Research 40 (2019): 43-84, mit: M. Dütsch, O. Struck.

Regional Mobility and Spatial Inequality: Determinants of Spatial Labor Market Behavior Considering Firm- and Region-Specific Factors, in: Spatial Research and Planning 76 (2018): 497514.

Regionale Mobilität am Arbeitsmarkt. Individuelle, betriebliche und wirtschaftsstrukturelle Determinanten von Mobilität und Einkommen, in: Kölner Zeitschrift für Soziologie und Sozialpsychologie 71 (2019): 181-210, mit: M. Dütsch, O. Struck.

Arbeitsplatzmobilität zwischen Ost-, Nord-, und Süddeutschland. Erfolgsfaktoren von Einkommenszuwächsen, erscheint in: Sozialer Fortschritt (2020), mit: M. Dütsch, O. Struck. 